

\title{
Well-posedness and smoothing effect for generalized nonlinear Schrödinger equations
}

Pierre-Yves Bienaimé, Abdesslam Boulkhemair

\section{To cite this version:}

Pierre-Yves Bienaimé, Abdesslam Boulkhemair. Well-posedness and smoothing effect for generalized nonlinear Schrödinger equations. 2017. hal-01515658v2

\section{HAL Id: hal-01515658 \\ https://hal.science/hal-01515658v2}

Preprint submitted on 20 Jun 2017

HAL is a multi-disciplinary open access archive for the deposit and dissemination of scientific research documents, whether they are published or not. The documents may come from teaching and research institutions in France or abroad, or from public or private research centers.
L'archive ouverte pluridisciplinaire HAL, est destinée au dépôt et à la diffusion de documents scientifiques de niveau recherche, publiés ou non, émanant des établissements d'enseignement et de recherche français ou étrangers, des laboratoires publics ou privés. 


\title{
Well-posedness and smoothing effect for generalized nonlinear Schrödinger equations
}

\author{
April 27, 2017 \\ Pierre-Yves Bienaimé and Abdesslam Boulkhemair \\ Laboratoire de Mathématiques Jean Leray, \\ CNRS UMR6629, Université de Nantes, \\ 2, rue de la Houssinière, BP 92208, 44322 Nantes, France. \\ e-mail : Pierre-Yves.Bienaime at univ-nantes.fr \\ boulkhemair-a at univ-nantes.fr
}

\begin{abstract}
We improve the result obtained by one of the authors, [Bie], and establish the well posed-ness of the Cauchy problem for some nonlinear equations of Schrödinger type in the usual Sobolev space $H^{s}\left(\mathbb{R}^{n}\right)$ for $s>\frac{n}{2}+2$ instead of $s>\frac{n}{2}+3$ in [Bie]. We also improve the smoothing effect of the solution and obtain the optimal exponent.
\end{abstract}

\section{Introduction}

Consider the following nonlinear Cauchy problem :

$$
\left\{\begin{array}{l}
\partial_{t} u=i \mathscr{L} u+F\left(u, \nabla_{x} u, \bar{u}, \nabla_{x} \bar{u}\right), \quad t \in \mathbb{R}, x \in \mathbb{R}^{n}, \\
u(x, 0)=u_{0}(x) \in H^{s}\left(\mathbb{R}^{n}\right),
\end{array}\right.
$$

where the function $F$ is sufficiently regular in $\mathbb{C} \times \mathbb{C}^{n} \times \mathbb{C} \times \mathbb{C}^{n}$, the operator $\mathscr{L}$ has the form

$$
\mathscr{L}=\sum_{j \leq j_{0}} \partial_{x_{j}}^{2}-\sum_{j>j_{0}} \partial_{x_{j}}^{2},
$$

with a fixed $j_{0} \in\{1,2, \ldots, n\}$, and $H^{s}\left(\mathbb{R}^{n}\right), s \in \mathbb{R}$, is the usual Sobolev space on $\mathbb{R}^{n}$. Thus, $\mathscr{L}$ generalizes the Laplace operator but is not elliptic unless $j_{0}=n$. Hence, such equations are generalizations of the nonlinear Schrödinger (NLS) equations.

In this paper, we continue the work undertaken in [Bie] and study the local existence and the smoothing effect of the solutions of the Cauchy problem (1) with essentially the following 
goal : to obtain the optimal index $s$ of regularity for which (1) is well posed. In fact, the partial differential equation being of second order and semi-linear the optimal condition on $s$ should be $s>\frac{n}{2}+1$. Unfortunately, up to now and due to issues that occur when estimating the remainder obtained after the linearization of the non linear equation, we have not been able to prove the wished result under such a condition. In any case, we shall return to this question in a future work. In this paper, we establish the following :

Theorem 1.1 Assume that $F$ vanishes to the third order at 0 , that is, $F$ and their partial derivatives up to the second order vanishes at 0 . Then, for every $s>\frac{n}{2}+2$ and every initial data $u_{0} \in H^{s}\left(\mathbb{R}^{n}\right)$, there exists a real number $T>0$ such that the Cauchy problem (1) has a unique solution $u$ which is defined on the interval $[0, T]$ and satisfies

$$
u \in C\left([0, T] ; H^{s}\left(\mathbb{R}^{n}\right)\right)
$$

and

$$
\left\|J^{s+\frac{1}{2}} u\right\|_{T} \stackrel{\text { def }}{=} \sup _{\mu \in \mathbb{Z}^{n}}\left(\int_{0}^{T} \int_{\mathbb{R}^{n}}\left|\langle x-\mu\rangle^{-\sigma_{0}} J^{s+\frac{1}{2}} u(x, t)\right|^{2} d x d t\right)^{\frac{1}{2}}<\infty,
$$

where $J=(1-\Delta)^{1 / 2}, \Delta=\sum_{k=1}^{k=n} \partial_{x_{k}}^{2}$ and $\sigma_{0}>\frac{1}{2}$ is fixed. Moreover, given a bounded subset $B$ of $H^{s}\left(\mathbb{R}^{n}\right)$, there exists a real number $T>0$ such that, for every $u_{0} \in B$, the associated solution $u$ of (1) exists on the interval $[0, T]$ and the map which associates $u$ to $u_{0}$ is Lipschitz continuous from $B$ into the space

$$
\left\{w \in C\left([0, T] ; H^{s}\left(\mathbb{R}^{n}\right)\right) ;\left\|J^{s+\frac{1}{2}} w\right\|_{T}<\infty\right\} .
$$

In [Bie], this theorem is proved under the assumption $s>\frac{n}{2}+3$. We also improve the result of [Bie] with respect to the smoothing effect of the solution since $\sigma_{0}=2$ there. Note that the assumption $\sigma_{0}>\frac{1}{2}$ in the above theorem seems to be sharp; we refer for example to the survey article [Rob] on the subject of Kato's smoothing effect. Recall that at the origin of [Bie] was the significant work of C. E. Kenig, G. Ponce and L. Vega, [KePoVe], who first studied (1) and established the local existence and the smoothing effect of the solutions assuming that $F$ is a polynomial and $s>s_{0}$, the index $s_{0}$ being sufficiently large. These authors also studied the case where $F$ (is a polynomial and) vanishes to the second order at 0 . However, it seems that in that case we need to work in weighted Sobolev spaces.

The Cauchy problem (1) has been extensively studied in the nineties mainly when $\mathscr{L}=$ $\Delta$, that is, in the case of the Schrödinger equation. See the references in $[\mathrm{KePoVe}]$. The case $\mathscr{L} \neq \Delta$ is less well known. Nevertheless, it is motivated by several equations coming from the applications such as Ishimori's type equations or Davey-Stewartson's type systems. For more details, we refer the reader to the very instructive introduction of [KePoVe]. Let us now quote some papers which are more or less related to this subject. In [KePoVe2], 2004, the authors extended their results of 1998 to the quasilinear case assuming essentially that the corresponding dispersive operator $\mathscr{L}$ is elliptic and non trapping. The non elliptic case is treated in [KePoRoVe1], 2005, and [KePoRoVe2], 2006. In [BeTa], 2008, the authors 
solved the Cauchy problem (1) for $s>\frac{n}{2}+1$ in modified Sobolev spaces and assuming $F\left(u, \nabla_{x} u, \bar{u}, \nabla_{x} \bar{u}\right)$ bilinear. More recently, in [MaMeTa1], 2012, and [MaMeTa2], 2014, the authors considered the quasilinear Schrödinger equation

$$
i \partial_{t} u+\sum_{j, k} g^{j, k}\left(u, \nabla_{x} u\right) \partial_{j} \partial_{k} u=F\left(u, \nabla_{x} u\right)
$$

and obtained the local well-posedness of the associated Cauchy problem for $s>\frac{n}{2}+3$ in the quadratic case (with modified Sobolev spaces) and for $s>\frac{n}{2}+\frac{5}{2}$ in the non quadratic case. However, they assume the smallness of the data and they do not seem to obtain the smoothing effect of the solutions.

The proof of Theorem 1.1 follows the same ideas as that of [KePoVe] or [Bie]. Of course, the general plan is unoriginal : linearization of the non linear equation, then, establishing energy estimates for solutions of the linear equation, and finally, solving the non linear equation by means of an appropriate fixed point theorem. Like [Bie], we start by applying a para-linearization, that is a linearization in the sense of Bony, [Bon], instead of the classical linearization. This leads us to the use of the para-differential calculus whose main interest lies in the fact that it eliminates the usual losses of regularity due to commutators. One obtains a para-linear equation and most of the proof of the theorem is concerned with the study of such an equation, that is, the well-posedness in the Sobolev spaces of the associated Cauchy problem by means of energy and smoothing effect estimates. As did Kenig, Ponce and Vega, we establish the smoothing effect estimate by using Doi's argument, [Doi], via Gårding's inequality, and we prove the energy estimates by following an idea of Takeuchi [Tak], that is, by constructing a non classical invertible pseudo-differential operator $\mathbf{C}$ which allows estimates for $\mathbf{C} u$ if $u$ is a solution of the para-linear equation. At last, we solve the non linear Cauchy problem (1) by applying these estimates to an integro-differential equation which is equivalent to (1) and obtain the solution as the fixed point of an appropriate contraction in an appropriate complete metric space.

Now, in order to give a more precise idea about our proof, let us indicate the differences with that given in [Bie]. In fact, there are three main differences :

- We simplify certain arguments of [Bie]; for example, we no longer need to use the general Hörmander symbol spaces $S_{\rho, \delta}^{m}$, we only use $S_{1,0}^{m}$ and $S_{0,0}^{m}$. Also, we only use the original para-differential operators (see Section 2) and not the variant introduced in [Bie].

- The linear theorem, that is, Theorem 3.1 (see Section 3), is proved for general paradifferential operators $T_{b_{1}}$ and $T_{b_{2}}$ of order 0 instead of para-multiplication operators. Note also that we allow the operators $C_{1}$ and $C_{2}$ to be abstract bounded operators.

- The third difference lies in the non linear part (see Section 4) and is crucial for our improvement of the result of [Bie] : we use anisotropic Sobolev spaces and an interpolation inequality (see Lemma 7.3) to estimate the remainder of the para-linearized equation. 


\section{Notations and preliminary results}

Some notations used in the paper :

- $J^{s}=(1-\Delta)^{s / 2}=\langle D\rangle^{s}$ is the operator whose symbol is $\langle\xi\rangle^{s}=\left(1+\xi^{2}\right)^{s / 2}$.

- $D_{x_{k}}=-i \partial_{x_{k}}, \quad D_{x}=-i \partial_{x}$.

$-|\alpha|=\sum_{j=1}^{j=n} \alpha_{j} \quad$ if $\quad \alpha \in \mathbb{N}^{n}$.

- $\Delta v=\left(\Delta v_{1}, \ldots, \Delta v_{n}\right)$ and $\nabla v=\left(\nabla v_{1}, \ldots, \nabla v_{n}\right) \quad$ if $\quad v=\left(v_{1}, \ldots, v_{n}\right)$.

- $\mathscr{S}\left(\mathbb{R}^{n}\right)$ denotes the Schwartz space of rapidly decreasing functions in $\mathbb{R}^{n}$.

— $\mathscr{D}\left(\mathbb{R}^{n}\right)$ denotes the space of smooth functions with compact support in $\mathbb{R}^{n}$.

- $\mathscr{D}^{\prime}\left(\mathbb{R}^{n}\right)$ denotes the space of distributions in $\mathbb{R}^{n}$.

- $\mathscr{S}^{\prime}\left(\mathbb{R}^{n}\right)$ denotes the space of tempered distributions in $\mathbb{R}^{n}$.

- $\hat{u}$ or $\mathscr{F}(u)$ denotes the Fourier transform of $u$.

- $H^{s}\left(\mathbb{R}^{n}\right)=\left\{u \in \mathscr{S}^{\prime}\left(\mathbb{R}^{n}\right) ;\langle\xi\rangle^{s} \hat{u} \in L^{2}\left(\mathbb{R}^{n}\right)\right\}$ is the usual Sobolev space of regularity $s$.

- $\|u\|_{s}=\left(\int_{\mathbb{R}^{n}}\langle\xi\rangle^{2 s}|\hat{u}(\xi)|^{2} d \xi\right)^{1 / 2}$ denotes the norm of $u$ in $H^{s}\left(\mathbb{R}^{n}\right)$.

- $\|u\|_{E}$ denotes the norm of $u$ in the space $E$.

- Hörmander's classes of symbols : If $m \in \mathbb{R}$ and $\gamma, \delta \in[0,1]$,

$$
S_{\gamma, \delta}^{m}=\left\{a \in C^{\infty}\left(\mathbb{R}^{n} \times \mathbb{R}^{n}\right) ; \forall \alpha, \beta \in \mathbb{N}^{n}, \quad\left|\partial_{x}^{\alpha} \partial_{\xi}^{\beta} a(x, \xi)\right| \leq A_{\alpha, \beta}\langle\xi\rangle^{m-\gamma|\beta|+\delta|\alpha|}\right\} .
$$

- If $\varrho>0$ is an integer, $C^{\varrho}\left(\mathbb{R}^{n}\right)$ denotes the set of functions in $\mathbb{R}^{n}$ which are bounded, of class $C^{m}$ and their derivatives up to $m$ are bounded. If $\varrho>0$ is not an integer, $C^{\varrho}\left(\mathbb{R}^{n}\right)$ denotes the Hölder class, that is, the set of $u$ in $C^{[\varrho]}\left(\mathbb{R}^{n}\right)$ such that

$$
\exists C \in \mathbb{R}, \forall(x, y) \in \mathbb{R}^{n} \times \mathbb{R}^{n},\left|\partial^{\alpha} u(x)-\partial^{\alpha} u(y)\right| \leq C|x-y|^{\varrho-[\varrho]} .
$$

— Op $S$ denotes the set of pseudodifferential operators whose symbols belong to $S$.

The following statement summarizes the pseudodifferential calculus associated to Hörmander's classes of symbols $S_{\gamma, \delta}^{m}$ :

Theorem 2.1 If $a \in S_{\gamma, \delta}^{m}, b \in S_{\gamma, \delta}^{m^{\prime}}, m, m^{\prime} \in \mathbb{R}$, and $0 \leq \delta<\gamma \leq 1$ or $0 \leq \delta \leq \gamma<1$, then :

(i) $a(x, D) b(x, D)=c(x, D)$ with $c \in S_{\gamma, \delta}^{m+m^{\prime}}$. Moreover,

$$
\begin{gathered}
c(x, \xi)=\int e^{-i y \cdot \eta} a(x, \xi+\eta) b(x+y, \xi) \frac{d y d \eta}{(2 \pi)^{n}} \\
=\sum_{|\nu|<N} \frac{1}{\nu !} \partial_{\xi}^{\nu} a(x, \xi) D_{x}^{\nu} b(x, \xi)+\sum_{|\nu|=N} \frac{1}{\nu !} \int_{0}^{1}(1-\theta)^{N-1} r_{\nu, \theta}(x, \xi) d \theta, \\
\text { where } r_{\nu, \theta}(x, \xi)=\int e^{-i y \cdot \eta} \partial_{\xi}^{\nu} a(x, \xi+\theta \eta) D_{x}^{\nu} b(x+y, \xi) \frac{d y d \eta}{(2 \pi)^{n}}
\end{gathered}
$$

and the $S_{\gamma, \delta}^{m+m^{\prime}-N(\gamma-\delta)}$ semi-norms of $r_{\nu, \theta}$ are bounded by products of semi-norms of $a$ and $b$ uniformly in $\theta \in[0,1]$. 
(ii) $a(x, D)^{*}=a^{*}(x, D)$ with $a^{*} \in S_{\gamma, \delta}^{m}$. Moreover,

$$
\begin{gathered}
a^{*}(x, \xi)=\int e^{-i y \cdot \eta} \bar{a}(x+y, \xi+\eta) \frac{d y d \eta}{(2 \pi)^{n}} \\
=\sum_{|\nu|<N} \frac{1}{\nu !} \partial_{\xi}^{\nu} D_{x}^{\nu} \bar{a}(x, \xi)+\sum_{|\nu|=N} \frac{1}{\nu !} \int_{0}^{1}(1-\theta)^{N-1} r_{\nu, \theta}^{*}(x, \xi) d \theta, \\
\text { where } r_{\nu, \theta}^{*}(x, \xi)=\int e^{-i y \cdot \eta} \partial_{\xi}^{\nu} D_{x}^{\nu} \bar{a}(x+y, \xi+\theta \eta) \frac{d y d \eta}{(2 \pi)^{n}},
\end{gathered}
$$

and the $S_{\gamma, \delta}^{m-N(\gamma-\delta)}$ semi-norms of $r_{\nu, \theta}^{*}$ are bounded by semi-norms of a uniformly in $\theta \in[0,1]$.

See [Tay], par exemple, for the proof. We shall also often need the following version of Calderón-Vaillancourt theorem :

Theorem 2.2 Let $a: \mathbb{R}^{n} \times \mathbb{R}^{n} \rightarrow \mathbb{C}$ be a bounded function. Assume that, for all $\alpha, \beta \in \mathbb{N}^{n}$ such that $|\alpha|+|\beta| \leq n+1$, there exists a constant $C_{\alpha, \beta}>0$ such that $\left|\partial_{x}^{\alpha} \partial_{\xi}^{\beta} a(x, \xi)\right| \leq$ $C_{\alpha, \beta}$ in $\mathbb{R}^{2 n}$. Then, the pseudodifferential operator $a(x, D)$ is bounded in $L^{2}\left(\mathbb{R}^{n}\right)$ and its operator norm is estimated by

$$
\sup _{|\alpha|+|\beta| \leq n+1}\left\|\partial_{x}^{\alpha} \partial_{\xi}^{\beta} a\right\|_{L^{\infty}}
$$

See $[\mathrm{CoMe}]$ for the proof.

The following technical lemma which is a consequence of Theorem 2.1 will be very useful in many of our proofs :

Lemma 2.1 Let $a \in S_{0,0}^{m}, m, \sigma \in \mathbb{R}$ and $\mu \in \mathbb{R}^{n}$. Then,

(i) we have $\langle x-\mu\rangle^{\sigma} a(x, D)\langle x-\mu\rangle^{-\sigma}=a_{\mu}(x, D)$, where $a_{\mu} \in S_{0,0}^{m}$ and the semi-norms of $a_{\mu}$ are bounded by semi-norms of a uniformly in $\mu$.

(ii) if $\sigma \geq 0$ and if, in addition, $a(x, \xi)$ is rapidly decreasing with respect to $x-\mu$, we have $\langle x-\mu\rangle^{\sigma} a(x, D)\langle x-\mu\rangle^{\sigma}=b_{\mu}(x, D)$, where $b_{\mu} \in S_{0,0}^{m}, b_{\mu}$ is also rapidly decreasing in $x-\mu$ and the semi-norms of $b_{\mu}$ are estimated uniformly in $\mu$ by expressions of the form

$$
\sup _{|\alpha|+|\beta| \leq N}\left\|\langle x-\mu\rangle^{2 \sigma}\langle\xi\rangle^{-m} \partial_{x}^{\alpha} D_{\xi}^{\beta} a\right\|_{L^{\infty}}
$$

Here, the fact that the symbol $a(x, \xi)$ is rapidly decreasing with respect to $x-\mu$ means that, for every integer $N$ and all multi-indices $\alpha, \beta$, the function $\langle x-\mu\rangle^{N}\langle\xi\rangle^{-m} \partial_{x}^{\alpha} D_{\xi}^{\beta} a$ is bounded in $\mathbb{R}^{n} \times \mathbb{R}^{n}$, and we shall often meet such symbols in this paper.

Proof : (i) When $\sigma \geq 0$, we can use Theorem 2.1(i) and integrations by parts to obtain

$$
\begin{gathered}
a_{\mu}(x, \xi)=\langle x-\mu\rangle^{\sigma}(2 \pi)^{-n} \int \mathrm{e}^{-i y \cdot \eta} a(x, \xi+\eta)\langle x+y-\mu\rangle^{-\sigma} d y d \eta \\
=\langle x-\mu\rangle^{\sigma}(2 \pi)^{-n} \int \mathrm{e}^{-i y \cdot \eta} J_{\eta}^{N}\left[\langle\eta\rangle^{-N} a(x, \xi+\eta)\right]\langle y\rangle^{-N} J_{y}^{N}\left[\langle x+y-\mu\rangle^{-\sigma}\right] d y d \eta
\end{gathered}
$$


where $N$ is a large and even integer. Hence, by taking derivatives and bounding, and next by applying Peetre's inequality,

$$
\begin{gathered}
\left|a_{\mu}(x, \xi)\right| \leq C\left\|\langle\xi\rangle^{-m} a\right\|_{C^{N}}\langle x-\mu\rangle^{\sigma} \int\langle\eta\rangle^{-N}\langle\xi+\eta\rangle^{m}\langle y\rangle^{-N}\langle x+y-\mu\rangle^{-\sigma} d y d \eta \\
\leq 2^{\frac{\sigma+|m|}{2}} C\left\|\langle\xi\rangle^{-m} a\right\|_{C^{N}}\langle\xi\rangle^{m} \int\langle\eta\rangle^{|m|-N}\langle y\rangle^{\sigma-N} d y d \eta=C^{\prime}\langle\xi\rangle^{m}\left\|\langle\xi\rangle^{-m} a\right\|_{C^{N}},
\end{gathered}
$$

where $C$ and $C^{\prime}$ are constants which are independent of $\mu$, and $N$ is taken for example such that $N \geq|m|+\sigma+n+1$. Of course, the derivatives of $a_{\mu}$ are treated in the same manner.

The case $\sigma<0$ follows from the preceding case by considering the adjoint

$$
a_{\mu}(x, D)^{*}=\langle x-\mu\rangle^{-\sigma} a(x, D)^{*}\langle x-\mu\rangle^{\sigma}
$$

and by applying Theorem 2.1(ii).

(ii) By using the formula in Theorem 2.1(ii) once more, it is easy to see that, if $a$ is rapidly decreasing with respect to $x-\mu$, then the symbol $a^{*}$ is also rapidly decreasing with respect to $x-\mu$ and that, for all $N \in \mathbb{N}, \alpha, \beta \in \mathbb{N}^{n}$, there exist $M \in \mathbb{N}$ and a non negative constant $C_{N, \alpha, \beta}$ which does not depend on $\mu$ such that

$$
\left\|\langle x-\mu\rangle^{N}\langle\xi\rangle^{-m} \partial_{x}^{\alpha} D_{\xi}^{\beta} a^{*}\right\|_{L^{\infty}} \leq C_{N, \alpha, \beta} \sup _{\left|\alpha^{\prime}\right|+\left|\beta^{\prime}\right| \leq M}\left\|\langle x-\mu\rangle^{N}\langle\xi\rangle^{-m} \partial_{x}^{\alpha^{\prime}} D_{\xi}^{\beta^{\prime}} a\right\|_{L^{\infty}} .
$$

Now, by following the same argument as that used in the first part, one can check that the same claim holds exactly when we replace $a^{*}$ by $a_{\mu}$ in the above assertion; in particular, we have the estimate

$$
\left\|\langle x-\mu\rangle^{N}\langle\xi\rangle^{-m} \partial_{x}^{\alpha} D_{\xi}^{\beta} a_{\mu}\right\|_{L^{\infty}} \leq C_{N, \alpha, \beta} \sup _{\left|\alpha^{\prime}\right|+\left|\beta^{\prime}\right| \leq M}\left\|\langle x-\mu\rangle^{N}\langle\xi\rangle^{-m} \partial_{x}^{\alpha^{\prime}} D_{\xi}^{\beta^{\prime}} a\right\|_{L^{\infty}}
$$

and since we can write obviously $b_{\mu}(x, \xi)=\langle x-\mu\rangle^{2 \sigma} a_{\mu}(x, \xi)$, this achieves the proof of the lemma.

When studying the non linear equation, the following result is important in order to explain the assumption made on the non-linearity $F$.

Lemma 2.2 For all $s \geq 0$ and all $\sigma>\frac{n}{2}$, there exists a constant $C>0$ such that, for all $v \in H^{s}\left(\mathbb{R}^{n}\right)$, the sequence $\mu \mapsto\left\|\langle x-\mu\rangle^{-\sigma} v\right\|_{s}$ is in $\ell^{2}\left(\mathbb{Z}^{n}\right)$ and

$$
\sum_{\mu}\left\|\langle x-\mu\rangle^{-\sigma} v\right\|_{s}^{2} \leq C\|v\|_{s}^{2}
$$

In particular, if $s>\frac{n}{2}, u, v \in H^{s}\left(\mathbb{R}^{n}\right)$ and $\chi$ is a smooth and rapidly decreasing function, then, $\mu \mapsto\|\chi(x-\mu) u v\|_{s}$ is in $\ell^{1}\left(\mathbb{Z}^{n}\right)$ and

$$
\sum_{\mu}\|\chi(x-\mu) u v\|_{s} \leq C\|u\|_{s}\|v\|_{s} .
$$


Proof: The case $s=0$ is obvious and follows from the fact that $\sum_{\mu}\langle x-\mu\rangle^{-2 \sigma}$ is a bounded function. The case where $s$ is a positive integer reduces to the case $s=0$ by taking derivatives via Leibniz formula. The general case is obtained by interpolation. Indeed, the map $v \mapsto\langle x-\mu\rangle^{-\sigma} v$ being linear and bounded from $H^{s}$ into $\ell^{2}\left(\mathbb{Z}^{n}, H^{s}\right)$ for integral indices $s=s_{1}, s_{2}$, it will be also bounded from $H^{s^{\prime}}$ into $\ell^{2}\left(\mathbb{Z}^{n}, H^{s^{\prime}}\right)$ for any real $s^{\prime}$ between $s_{1}$ and $s_{2}$. This follows from the fact that

$$
\left[\ell^{2}\left(\mathbb{Z}^{n}, H^{s_{1}}\right), \ell^{2}\left(\mathbb{Z}^{n}, H^{s_{2}}\right)\right]_{\theta}=\ell^{2}\left(\mathbb{Z}^{n},\left[H^{s_{1}}, H^{s_{2}}\right]_{\theta}\right),
$$

for $0<\theta<1$. See for example [BeLö], Theorem 5.1.2, page 107 .

The second part is a consequence of the first one and the fact that $H^{s}\left(\mathbb{R}^{n}\right)$ is an algebra if $s>\frac{n}{2}$.

Let us now recall some results on paradifferential operators.

Definition 2.1 We define the class $\Sigma_{\varrho}^{m}$ where $m \in \mathbb{R}$ and $\varrho \geq 0$ to be the class of symbols $a(x, \xi)$ defined on $\mathbb{R}^{n} \times \mathbb{R}^{n}$ which are $C^{\infty}$ in $\xi$ and $C^{\varrho}$ in $x$, in the sense that

$$
\forall \alpha \in \mathbb{N}^{n},\left|\partial_{\xi}^{\alpha} a(x, \xi)\right|\langle\xi\rangle^{-m+|\alpha|} \in C^{\varrho}\left(\mathbb{R}^{n} \times \mathbb{R}^{n}\right),
$$

$C^{\varrho}$ being replaced by $L^{\infty}$ when $\varrho=0$. If $a \in \Sigma_{\varrho}^{m}, m$ is the order of $a$ and $\varrho$ is its regularity. Following J.-M. Bony, we associate to a symbol $a$ in $\Sigma_{\varrho}^{m}$ the paradifferential operator $T_{a, \chi}$ defined by the expression

$$
\widehat{T_{a, \chi} u}(\xi)=(2 \pi)^{-n} \int_{\mathbb{R}^{n}} \chi(\xi-\eta, \eta) \mathscr{F}_{1}(a)(\xi-\eta, \eta) \hat{u}(\eta) d \eta,
$$

where $\chi$ is what one calls a para-truncature, that is a $C^{\infty}$ function in $\mathbb{R}^{n} \times \mathbb{R}^{n}$ satisfying the following properties :

(i) $\exists \varepsilon>0$ such that $\varepsilon<1$ and $\chi(\xi, \eta)=0$ if $|\xi| \geq \varepsilon|\eta|, \xi, \eta \in \mathbb{R}^{n}$.

(ii) $\exists \varepsilon^{\prime}>0, \varepsilon^{\prime \prime}>0$, such that $\varepsilon^{\prime}<\varepsilon$ and $\chi(\xi, \eta)=1$ if $|\xi| \leq \varepsilon^{\prime}|\eta|$ and $|\eta| \geq \varepsilon^{\prime \prime}$.

(iii) $\forall \alpha \in \mathbb{N}^{2 n}, \quad \exists A_{\alpha}>0, \quad \forall \zeta \in \mathbb{R}^{2 n}, \quad\langle\zeta\rangle^{|\alpha|}\left|\partial^{\alpha} \chi(\zeta)\right| \leq A_{\alpha}$.

The first important result on paradifferential operators is that, even if one can show that $T_{a, \chi}=\tilde{a}(x, D)$ with some $\tilde{a} \in S_{1,1}^{m}$, they are bounded in the Sobolev spaces in the usual manner. In fact, we have :

Theorem 2.3 Assume that $\chi$ satisfies only the first and third property among the above ones. Then, for every real $s, T_{a, \chi}$ is bounded from $H^{s}\left(\mathbb{R}^{n}\right)$ into $H^{s-m}\left(\mathbb{R}^{n}\right)$ and its operator norm is estimated by a semi-norm of $a$ in $\Sigma_{\varrho}^{m}$. In particular, if $a=a(x) \in L^{\infty}\left(\mathbb{R}^{n}\right)$, then, for every real $s, T_{a, \chi}$ is bounded in $H^{s}\left(\mathbb{R}^{n}\right)$ with an operator norm bounded by a constant times $\|a\|_{L^{\infty}}$.

Proof : See [Bon], [Me2] or [Tay].

Concerning the dependence with respect to the para-truncature $\chi$, one can say the following : 
Theorem 2.4 If $\varrho>0$ and $\chi_{1}, \chi_{2}$ are para-truncatures, then, the operator $T_{a, \chi_{1}}-T_{a, \chi_{2}}$ is bounded from $H^{s}\left(\mathbb{R}^{n}\right)$ into $H^{s-m+\varrho}\left(\mathbb{R}^{n}\right)$ and its operator norm is estimated by a semi-norm of $a$ in $\Sigma_{\varrho}^{m}$.

Proof : See [Bon], [Me2] or [Tay].

This result shows that the dependance of $T_{a, \chi}$ on $\chi$ is less important than that on $a$. It also explains why the remainders in the paradifferential theory are only $\varrho$-regularizing. From now on, we shall write $T_{a}$ instead of $T_{a, \chi}$ unless it is needed.

Note also that a possible choice of the para-truncature that we shall often use in the sequel is given by

$$
\chi(\xi, \eta)=\chi_{1}(\xi /|\eta|)\left(1-\psi_{1}(\eta)\right),
$$

where $\psi_{1}, \chi_{1} \in C^{\infty}\left(\mathbb{R}^{n}\right), \psi_{1}=1$ in a neighbourhood of $0, \psi_{1}=0$ out of $B\left(0, \varepsilon^{\prime \prime}\right)$, and $\chi_{1}=1$ on $B\left(0, \varepsilon^{\prime}\right), \operatorname{supp}(\chi) \subset B(0, \varepsilon)$, with $\varepsilon$ and $\varepsilon^{\prime}$ satisfying $0<\varepsilon^{\prime}<\varepsilon<1$. In this case, $T_{a, \chi}=\tilde{a}(x, D)$ with the following expression of $\tilde{a}$ :

$$
\tilde{a}(x, \xi)=\left(1-\psi_{1}(\xi)\right)|\xi|^{n} \int_{\mathbb{R}^{n}} \mathscr{F}^{-1}\left(\chi_{1}\right)(|\xi|(x-y)) a(y, \xi) d y .
$$

The following lemma gives some properties of $\tilde{a}$ which will be needed in the sequel and often used implicitly.

Lemma 2.3 Let $\varrho \geq 0$ and $a \in \Sigma_{\varrho}^{m}$. Then, $\tilde{a}$ is smooth and

$$
\begin{gathered}
\left|\partial_{\xi}^{\beta} \partial_{x}^{\alpha} \tilde{a}(x, \xi)\right| \leq A_{\alpha, \beta}\langle\xi\rangle^{m-|\beta|} \quad \text { if } \quad|\alpha| \leq \varrho, \\
\left|\partial_{\xi}^{\beta} \partial_{x}^{\alpha} \tilde{a}(x, \xi)\right| \leq A_{\alpha, \beta}\langle\xi\rangle^{m-|\beta|+|\alpha|-\varrho} \quad \text { if } \quad|\alpha|>\varrho,
\end{gathered}
$$

where $A_{\alpha, \beta}$ are non negative constants; more precisely, the $A_{\alpha, \beta}$ can be estimated by seminorms of a in $\Sigma_{\varrho}^{m}$. In particular, $\tilde{a} \in S_{1,1}^{m}$.

Moreover, if $\theta$ is a smooth function with support in some compact subset of $\mathbb{R}^{n}$ and $\theta_{\mu}(x)=\theta(x-\mu), \mu \in \mathbb{Z}^{n}$, then, for all $N \in \mathbb{N}$, we have

$$
\begin{gathered}
\langle x-\mu\rangle^{N}\left|\partial_{\xi}^{\beta} \partial_{x}^{\alpha} \widetilde{\theta_{\mu} a}(x, \xi)\right| \leq A_{\alpha, \beta, N}\langle\xi\rangle^{m-|\beta|} \quad \text { if } \quad|\alpha| \leq \varrho, \\
\langle x-\mu\rangle^{N}\left|\partial_{\xi}^{\beta} \partial_{x}^{\alpha} \widetilde{\theta_{\mu} a}(x, \xi)\right| \leq A_{\alpha, \beta, N}\langle\xi\rangle^{m-|\beta|+|\alpha|-\varrho} \quad \text { if } \quad|\alpha|>\varrho,
\end{gathered}
$$

where the $A_{\alpha, \beta, N}$ do not depend on $\mu$ and are estimated by semi-norms of a in $\Sigma_{\varrho}^{m}$.

Proof : For the first part we refer to [Me2] or [Tay]. The second part follows from the first one by using for example for even $N$ the decomposition

$$
\langle x-\mu\rangle^{N}=\sum_{\alpha} \frac{(x-y)^{\alpha}}{\alpha !} \partial_{y}^{\alpha}\langle y-\mu\rangle^{N}
$$

together with the expression (2).

When dealing with non linear terms, we shall frequently use the following classical result : 
Proposition 2.1 If $F$ is a $C^{\infty}$ (or sufficiently regular) function in $\mathbb{C}^{m}, F(0)=0$ and $u_{1}, \ldots, u_{m}$ are functions in $H^{s}\left(\mathbb{R}^{n}\right), s>\frac{n}{2}$, then, $F\left(u_{1}, \ldots, u_{m}\right) \in H^{s}\left(\mathbb{R}^{n}\right)$ and we have precisely

$$
\left\|F\left(u_{1}, \ldots, u_{m}\right)\right\|_{s} \leq C\left(\left\|\left(u_{1}, \ldots, u_{m}\right)\right\|_{L^{\infty}}\right)\left\|\left(u_{1}, \ldots, u_{m}\right)\right\|_{s},
$$

where $\xi \mapsto C(\xi)$ is a non negative and non decreasing function.

An important property of the paradifferential operators consists in the fact that they are necessary to write down Bony's linearization formula, a formula that we recall here.

Theorem 2.5 (Bony's linearization formula) For all real functions $u_{1}, \ldots, u_{m} \in$ $H^{\frac{n}{2}+\varrho}\left(\mathbb{R}^{n}\right), \varrho>0$, and every function $F$ of $m$ real variables which is $C^{\infty}$ (or sufficiently regular) and vanishes in 0 , we have

$$
F\left(u_{1}, \ldots, u_{m}\right)=\sum_{i=1}^{i=m} T_{\partial_{u_{i}} F} u_{i}+r \quad \text { with } \quad r \in H^{\frac{n}{2}+2 \varrho}\left(\mathbb{R}^{n}\right) .
$$

Proof : See [Bon], [Me2] or [Mey].

The remainder $r$ in the above formula depends of course on $\left(u_{1}, \ldots, u_{m}\right)$. The following result essentially shows that $r$ is a locally Lipschitz function of $\left(u_{1}, \ldots, u_{m}\right)$. More precisely :

Theorem 2.6 If $u=\left(u_{1}, \ldots, u_{m}\right) \in H^{s}\left(\mathbb{R}^{n}, \mathbb{R}^{m}\right), s=\frac{n}{2}+\varrho, \varrho>0$, let us denote by $r(u)$ the remainder in Bony's formula. For all $u, v \in H^{s}\left(\mathbb{R}^{n}, \mathbb{R}^{m}\right)$, we have then

$$
\|r(u)-r(v)\|_{s+\varrho} \leq \theta\left(\|u\|_{s},\|v\|_{s}\right)\|u-v\|_{s},
$$

where $\theta\left(\|u\|_{s},\|v\|_{s}\right)$ is bounded if $u$ and $v$ vary in a bounded subset of $H^{s}\left(\mathbb{R}^{n}, \mathbb{R}^{m}\right)$.

Proof : See [Bie].

Remark : In the case of our equation, that is (1), even if $u$ has complex values, we shall be able to apply Bony's formula to the non linear expression $F(u, \nabla u, \bar{u}, \nabla \bar{u})$ where $u \in$ $H^{\frac{n}{2}+1+\varrho}\left(\mathbb{R}^{n}\right)$. Indeed, we can write

$$
F(u, \nabla u, \bar{u}, \nabla \bar{u})=G(\operatorname{Re}(u), \nabla R e(u), \operatorname{Im}(u), \nabla \operatorname{Im}(u))
$$

where $G\left(x_{1}, x_{2}, y_{1}, y_{2}\right)=F\left(x_{1}+i y_{1}, x_{2}+i y_{2}, x_{1}-i y_{1}, x_{2}-i y_{2}\right)$ which is a function from $\mathbb{R}^{2 n+2}$ into $\mathbb{C}$. We apply then Bony's formula to $G$ and obtain that

$$
F(u, \nabla u, \bar{u}, \nabla \bar{u})=T_{\partial_{x_{1}} G} \operatorname{Re}(u)+T_{\partial_{x_{2}} G} \nabla \operatorname{Re}(u)+T_{\partial_{y_{1}} G} \operatorname{Im}(u)+T_{\partial_{y_{2}} G} \nabla \operatorname{Im}(u)+r(u) .
$$

At last, by using the fact that $\operatorname{Re}(u)=\frac{u+\bar{u}}{2}, \operatorname{Im}(u)=\frac{u-\bar{u}}{2 i}, \partial_{z}=\frac{1}{2}\left(\partial_{x}-i \partial_{y}\right)$ and $\partial_{\bar{z}}=$ $\frac{1}{2}\left(\partial_{x}+i \partial_{y}\right)$, and then the linearity of $T_{b}$ with respect to $b$, we obtain the formula used in this paper :

$$
F(u, \bar{u}, \nabla u, \nabla \bar{u})=T_{\partial_{u} F} u+T_{\partial_{\bar{u}} F} \bar{u}+T_{\partial_{\nabla u} F} \nabla u+T_{\partial_{\nabla \bar{u}} F} \nabla \bar{u}+r(u)
$$

with $r(u) \in H^{\frac{n}{2}+2 \varrho}\left(\mathbb{R}^{n}\right)$ if $u \in H^{\frac{n}{2}+1+\varrho}\left(\mathbb{R}^{n}\right)$.

We shall also often need the following result similar to Lemma 2.1 : 
Lemma 2.4 Let $a \in \Sigma_{0}^{0}\left(\mathbb{R}^{n}\right), \theta \in \mathscr{D}\left(\mathbb{R}^{n}\right), \theta_{\mu}(x)=\theta(x-\mu), \mu \in \mathbb{R}^{n}$ and $s \in \mathbb{R}$, and consider the paradifferential operator $T_{\theta_{\mu} a}=T_{\theta_{\mu} a, \chi}$ (where the para-truncature $\chi$ does not necessarily satisfy the second property of Definition 2.1). Then, for all $\sigma \geq 0$, the operator $\langle x-\mu\rangle^{\sigma} T_{\theta_{\mu} a}\langle x-\mu\rangle^{\sigma}$ is bounded in $H^{s}\left(\mathbb{R}^{n}\right)$ and there exist $N \in \mathbb{N}$ and a non negative constant $C$ such that, for every $\mu \in \mathbb{R}^{n}$,

$$
\left\|\langle x-\mu\rangle^{\sigma} T_{\theta_{\mu} a}\langle x-\mu\rangle^{\sigma}\right\|_{\mathcal{L}\left(H^{s}\right)} \leq C \sup _{|\alpha| \leq N}\left\|\langle\xi\rangle^{|\alpha|} \partial_{\xi}^{\alpha} a\right\|_{L^{\infty}} .
$$

Proof : First, one can assume that $\sigma$ is an integer and even an even integer. Let us denote by $a_{\mu}$ the symbol $\theta_{\mu} a$ and consider first the operator $T_{a_{\mu}}\langle x-\mu\rangle^{\sigma}$. Recall that $T_{a_{\mu}}=\tilde{a}_{\mu}(x, D)$ with

$$
\tilde{a}_{\mu}(x, \xi)=\left(1-\psi_{1}(\xi)\right)|\xi|^{n} \int_{\mathbb{R}^{n}} \mathscr{F}^{-1}\left(\chi_{1}\right)(|\xi|(x-y)) a_{\mu}(y, \xi) d y
$$

where $\psi_{1}, \chi_{1} \in C^{\infty}\left(\mathbb{R}^{n}\right), \psi_{1}=1$ in a neighbourhood of $0, \psi_{1}=0$ out of $B\left(0, \varepsilon^{\prime \prime}\right)$, and $\chi_{1}=1$ on $B\left(0, \varepsilon^{\prime}\right), \operatorname{supp}(\chi) \subset B(0, \varepsilon)$, with $\varepsilon$ and $\varepsilon^{\prime}$ satisfying $0<\varepsilon^{\prime}<\varepsilon<1$. Hence, we can write for arbitrary $u \in \mathscr{S}\left(\mathbb{R}^{n}\right)$,

$$
\begin{gathered}
T_{a_{\mu}}\langle x-\mu\rangle^{\sigma} u(x)=(2 \pi)^{-n} \int \mathrm{e}^{i x \xi} \tilde{a}_{\mu}(x, \xi) \mathscr{F}\left(\langle x-\mu\rangle^{\sigma} u\right)(\xi) d \xi \\
=(2 \pi)^{-n} \int \mathrm{e}^{i x \xi} \tilde{a}_{\mu}(x, \xi)\left\langle D_{\xi}+\mu\right\rangle^{\sigma} \hat{u}(\xi) d \xi=(2 \pi)^{-n} \int\left\langle D_{\xi}-\mu\right\rangle^{\sigma}\left[\mathrm{e}^{i x \xi} \tilde{a}_{\mu}(x, \xi)\right] \hat{u}(\xi) d \xi \\
=(2 \pi)^{-n} \sum_{\alpha} \frac{1}{\alpha !} D_{x}^{\alpha}\left[\langle x-\mu\rangle^{\sigma}\right] \int \mathrm{e}^{i x \xi} \partial_{\xi}^{\alpha} \tilde{a}_{\mu}(x, \xi) \hat{u}(\xi) d \xi
\end{gathered}
$$

where we have applied integrations by parts and Leibniz formula. So, we have proved that

$$
T_{a_{\mu}}\langle x-\mu\rangle^{\sigma}=\sum_{\alpha} \frac{1}{\alpha !} D_{x}^{\alpha}\left[\langle x-\mu\rangle^{\sigma}\right]\left(\partial_{\xi}^{\alpha} \tilde{a}_{\mu}\right)(x, D),
$$

where the sum is of course finite. Now, let us consider the operator $\left(\partial_{\xi}^{\alpha} \tilde{a}_{\mu}\right)(x, D)$ and let us remark that, for example,

$$
\begin{gathered}
\partial_{\xi_{k}} \tilde{a}_{\mu}(x, \xi)=\left(1-\psi_{1}(\xi)\right)|\xi|^{n} \int_{\mathbb{R}^{n}} \mathscr{F}^{-1}\left(\chi_{1}\right)(|\xi|(x-y)) \partial_{\xi_{k}} a_{\mu}(y, \xi) d y \\
-\left(1-\psi_{1}(\xi)\right)|\xi|^{n} \int_{\mathbb{R}^{n}} \mathscr{F}^{-1}\left(\chi_{2}\right)(|\xi|(x-y)) a_{\mu}(y, \xi) \frac{\xi_{k}}{|\xi|^{2}} d y \\
-\partial_{k} \psi_{1}(\xi)|\xi|^{n} \int_{\mathbb{R}^{n}} \mathscr{F}^{-1}\left(\chi_{1}\right)(|\xi|(x-y)) a_{\mu}(y, \xi) d y,
\end{gathered}
$$

where $\chi_{2}(\eta)=\sum_{j=1}^{n} \eta_{j} \partial_{j} \chi_{1}(\eta)$. This shows that

$$
\left(\partial_{\xi_{k}} \tilde{a}_{\mu}\right)(x, D)=\sum_{l=1}^{3} T_{\theta_{\mu} a^{l}, \chi^{l}}
$$

where the $a^{l}$ are symbols in $\Sigma_{0}^{-1}$ and the $\chi^{l}$ are para-truncatures which satisfy the first and third properties of Definition 2.1. By induction, $\left(\partial_{\xi}^{\alpha} \tilde{a}_{\mu}\right)(x, D)$ is then a finite sum of operators 
of the same form as $T_{a_{\mu}}=T_{a_{\mu}, \chi}$ (of order $\leq-|\alpha|$ ), and note also that the semi-norms of the associated symbols are bounded uniformly in $\mu$ by a semi-norm of $a$. Hence, $T_{a_{\mu}}\langle x-\mu\rangle^{\sigma}$ is a finite sum of operators of the form $P(x-\mu) T_{a_{\mu}}$, where $P$ is a polynomial (of degree $\leq \sigma)$, and consequently the problem is reduced to the study of the operator $\langle x-\mu\rangle^{\sigma} T_{a_{\mu}}$ only. Now, the symbol of the latter can be written as

$$
\begin{gathered}
\langle x-\mu\rangle^{\sigma} \tilde{a}_{\mu}(x, \xi)=\sum_{|\alpha| \leq \sigma} \frac{1}{\alpha !}\left(1-\psi_{1}(\xi)\right)|\xi|^{n} \int_{\mathbb{R}^{n}}(x-y)^{\alpha} \mathscr{F}^{-1}\left(\chi_{1}\right)(|\xi|(x-y)) \partial_{y}^{\alpha}\left[\langle y-\mu\rangle^{\sigma}\right] a_{\mu}(y, \xi) d y \\
=\sum_{|\alpha| \leq L} \frac{1}{\alpha !}\left(1-\psi_{1}(\xi)\right)|\xi|^{n} \int_{\mathbb{R}^{n}} \mathscr{F}^{-1}\left(\chi_{1}^{\alpha}\right)(|\xi|(x-y)) \theta^{\alpha}(y-\mu) a^{\alpha}(y, \xi) d y
\end{gathered}
$$

where $\chi_{1}^{\alpha}$ and $\theta^{\alpha}$ are similar to $\chi_{1}$ and $\theta$ respectively, and $a^{\alpha} \in \Sigma_{0}^{-|\alpha|}$ with semi-norms bounded by those of $a$. Hence, $\langle x-\mu\rangle^{\sigma} T_{a_{\mu}}$ is a finite sum of operators of the same form as $T_{a_{\mu}}$ whose symbols have semi-norms bounded uniformly in $\mu$ by a semi-norm of $a$. Eventually, the lemma follows from Theorem 2.3.

Let us also recall the Gårding's inequality which will be used crucially to prove the smoothing effect estimate.

Theorem 2.7 (Sharp Gårding's inequality for systems) Let $a(x, \xi)$ be a $k \times k$ matrix whose elements are in $S_{1,0}^{m}$ and which satisfies

$$
\left\langle\left(a(x, \xi)+a^{*}(x, \xi)\right) \zeta, \zeta\right\rangle \geq 0
$$

for all $\zeta \in \mathbb{C}^{k}$ and all $(x, \xi)$ such that $|\xi| \geq A_{0}$, where $a^{*}$ denotes the adjoint matrix of $a$ and $\langle.,$.$\rangle is the usual hermitian scalar product of \mathbb{C}^{k}$. Then, there exist a non negative constant $A$ and an integer $N$ such that, for all $u \in \mathscr{S}\left(\mathbb{R}^{n}, \mathbb{C}^{k}\right)$, we have

$$
\mathscr{R} e\langle a(x, D) u, u\rangle \geq-A \sup _{|\alpha|+|\beta| \leq N}\left\|\langle\xi\rangle^{|\beta|-m} \partial_{x}^{\alpha} \partial_{\xi}^{\beta} a\right\|_{L^{\infty}}\|u\|_{\frac{m-1}{2}}^{2}
$$

where $A$ depends only on $n, k$ and $A_{0}$.

Proof : See [Tay] or [Tat] for example.

\section{The paralinear equation}

In this section, we solve the Cauchy problem for the paralinear equation, that is, the linear equation obtained from (1) by applying Bony's linearization formula (Theorem 2.5).

Recall that $Q_{\mu}$ is the cube $\mu+[0,1]^{n}, \mu \in \mathbb{Z}^{n}$ and that $Q_{\mu}^{*}$ is a larger cube with side length 2 , for example, $\mu+[-1 / 2,3 / 2]^{n}$.

Theorem 3.1 Given $s \in \mathbb{R}$, consider the following linear Cauchy problem :

$$
\left\{\begin{array}{l}
\partial_{t} u=i \mathscr{L} u+T_{b_{1}} \cdot \nabla_{x} u+T_{b_{2}} \cdot \nabla_{x} \bar{u}+C_{1} u+C_{2} \bar{u}+f(x, t) \\
u(x, 0)=u_{0} \in H^{s}\left(\mathbb{R}^{n}\right)
\end{array}\right.
$$


We assume that $C_{1}$ and $C_{2}$ are bounded operators in $H^{s}\left(\mathbb{R}^{n}\right)$ and in $H^{s+2}\left(\mathbb{R}^{n}\right)$, that $b_{1}, b_{2} \in$ $\Sigma_{\varrho}^{m}, \varrho>0$, and more precisely that

$$
\left\{\begin{array}{l}
b_{k}(x, \xi)=\sum_{\mu \in \mathbb{Z}^{n}} \alpha_{k, \mu} \varphi_{k, \mu}(x, \xi), \quad \sum_{\mu}\left|\alpha_{k, \mu}\right| \leq A_{k}, \quad k=1,2 \\
\operatorname{supp}\left(x \mapsto \varphi_{k, \mu}(x, \xi)\right) \subseteq Q_{\mu}^{*}, \quad \sup _{|\beta| \leq N_{0}}\left\|\langle\xi\rangle^{|\beta|} \partial_{\xi}^{\beta} \varphi_{k, \mu}\right\|_{C} \leq 1,
\end{array}\right.
$$

and $\left\|C_{k}\right\|_{\mathcal{L}\left(H^{s}\right)},\left\|C_{k}\right\|_{\mathcal{L}\left(H^{s+2}\right)} \leq A_{k}, k=1,2, N_{0}$ being a large and fixed integer. We further assume that $b_{2}(x, \xi)$ is even in $\xi$. Then, the problem (8) has a unique solution $u$ which is in $C\left(\mathbb{R}, H^{s}\left(\mathbb{R}^{n}\right)\right)$ and satisfies, for all $T>0$,

$$
\begin{gathered}
\sup _{-T \leq t \leq T}\|u(t)\|_{s}^{2} \leq A\left(\left\|u_{0}\right\|_{s}^{2}+I_{T}\left(J^{s} f, J^{s} u\right)\right), \\
\left\|J^{s+\frac{1}{2}} u\right\|_{T}^{2} \leq A\left(\left\|u_{0}\right\|_{s}^{2}+I_{T}\left(J^{s} f, J^{s} u\right)\right),
\end{gathered}
$$

where the constant $A$ depends only on $n, s, \varrho, T, A_{1}$, and $A_{2}$, and the expression $I_{T}(v, w)$ is a finite sum of terms of the form

$$
\sup _{\mu \in \mathbb{Z}^{n}} \int_{-T}^{T}\left|\left\langle G_{\mu} v, w\right\rangle\right| d t
$$

with $G_{\mu} \in \mathrm{Op} S_{0,0}^{0}$ and the semi-norms of its symbol (up to $N_{0}$ ) are uniformly bounded by a constant that depends only on $s, n, \varrho, A_{1}$ and $A_{2}$.

Recall that $\|u\|_{T}^{2}=\sup _{\mu} \int_{-T}^{T} \int_{\mathbb{R}^{n}}\langle x-\mu\rangle^{-2 \sigma_{0}}|u(x, t)|^{2} d t d x$, where $\sigma_{0}>\frac{1}{2}$ is fixed.

Proof : Let us start by noting that the uniqueness is an obvious matter. Indeed, if $u_{1}$ and $u_{2}$ are solutions of (8), then, $u_{1}-u_{2}$ is a solution of (8) with $u_{0}=0$ and $f=0$, and the conclusion follows from (10).

As for the existence, as is customary with linear differential equations, it will follow from the a priori estimates (10) and (11) by using more or less standard arguments of functional analysis, and the proof of Theorem 3.1 will consist essentially in establishing them.

Another useful remark is that it will be sufficient to prove the theorem in $C\left(\mathbb{R}_{+}, H^{s}\left(\mathbb{R}^{n}\right)\right)$ instead of $C\left(\mathbb{R}, H^{s}\left(\mathbb{R}^{n}\right)\right)$ and the estimates (10) and (11) on $[0, T]$ instead of $[-T, T]$. In fact, if the theorem is proved on $\mathbb{R}_{+}$, one can apply it to $v(t)=u(-t)$ which satisfies a Cauchy problem of the same type as (8). The result is then that $v(-t)$ will extend $u$ to $\mathbb{R}_{-}$and satisfy (8) on $\mathbb{R}_{-}$, in addition to the fact that the estimates (10) and (11) are also extended to $[-T, 0]$.

So, let us assume that $u \in C\left([0, T] ; H^{s}\left(\mathbb{R}^{n}\right)\right)$ is a solution of the Cauchy problem (8).

In what follows, it will be quite convenient to use the following notation

$$
\nu_{N}(\varphi)=\sup _{1 \leq j \leq N} \sup _{|\alpha|+|\beta| \leq N}\left\|\langle\xi\rangle^{|\beta|} \partial_{x}^{\alpha} \partial_{\xi}^{\beta} \varphi\right\|_{L^{\infty}}^{j}
$$

and note that such a quantity is not a norm in general but it is well defined for $\varphi \in S_{1,0}^{0}$. Note also that, if $M \geq 1, \nu_{N}(\varphi)^{M} \leq \nu_{N M}(\varphi)$, a remark that will be often used implicitly.

In fact, the inequalities (10) and (11) will be deduced from the following ones: 
Proposition 3.1 Assume that the functions $\varphi_{k, \mu}$ defining the $b_{k}$ are $C^{\infty}$, that is, $\varphi_{k, \mu} \in S_{1,0}^{0}$, $k=1,2$. Then, there exist a positive real number $A$ and an integer $N$ such that, for all $R \geq 1$, there exists a pseudodifferential operator $\mathbf{C} \in \mathrm{Op} S_{0,0}^{0}$ such that, for all $T>0$, any solution $u \in C\left([0, T] ; H^{s}\left(\mathbb{R}^{n}\right)\right)$ of the Cauchy problem (8) satisfies

$$
\begin{aligned}
\sup _{0 \leq t \leq T}\|\mathbf{C} u(t)\|_{s}^{2} \leq & \left\|\mathbf{C} u_{0}\right\|_{s}^{2}+2 \int_{0}^{T}\left|\left\langle\mathbf{C} J^{s} f, \mathbf{C} J^{s} u\right\rangle\right| d t \\
& +A \sup _{k, \mu} \nu_{N}\left(\varphi_{k, \mu}\right)\left(R T \sup _{0 \leq t \leq T}\|u(t)\|_{s}^{2}+\frac{1}{R}\left\|J^{s+\frac{1}{2}} u\right\|_{T}^{2}\right) .
\end{aligned}
$$

Moreover, regarding the operator $\mathbf{C}$, we have the following precise bounds, for $v \in H^{s}\left(\mathbb{R}^{n}\right)$ :

$$
\begin{gathered}
\|\mathbf{C} v\|_{s} \leq A \sup _{\mu} \nu_{N}\left(\varphi_{1, \mu}\right)\|v\|_{s}, \\
\|v\|_{s} \leq A \sup _{\mu} \nu_{N}\left(\varphi_{1, \mu}\right)\|\mathbf{C} v\|_{s}+\frac{A}{R} \sup _{\mu} \nu_{N}\left(\varphi_{1, \mu}\right)^{2}\|v\|_{s} .
\end{gathered}
$$

Proposition 3.2 Under the same assumptions as above and with the same elements $A, R$, C and $N$, there exist also pseudodifferential operators $\psi_{j}(x, D) \in \mathrm{Op} S_{1,0}^{0}, j=1,2,3,4$, such that, for all $T>0$, any solution $u \in C\left([0, T] ; H^{s}\left(\mathbb{R}^{n}\right)\right)$ of the Cauchy problem (8) satisfies

$\left\|J^{s+\frac{1}{2}} u\right\|_{T}^{2} \leq A\left(1+T+T \sup _{\mu, k} \nu_{N}\left(\varphi_{k, \mu}\right)\right) \sup _{[0, T]}\|u\|_{s}^{2}+A \sum_{j=1}^{4} \sup _{\mu \in \mathbb{Z}^{n}} \int_{0}^{T}\left|\left\langle\psi_{j}(x-\mu, D) J^{s} f, J^{s} u\right\rangle\right| d t$

and

$$
\begin{aligned}
\left\|J^{s+\frac{1}{2}} \mathbf{C} u\right\|_{T}^{2} \leq A\left(1+T+T \sup _{\mu, k} \nu_{N}\left(\varphi_{k, \mu}\right)\right) & \sup _{[0, T]}\|\mathbf{C} u\|_{s}^{2}+A \sum_{j=1}^{4} \sup _{\mu} \int_{0}^{T}\left|\left\langle\psi_{j}(x-\mu, D) \mathbf{C} J^{s} f, \mathbf{C} J^{s} u\right\rangle\right| d t \\
& +A \sup _{k, \mu} \nu_{N}\left(\varphi_{k, \mu}\right)\left(R T \sup _{0 \leq t \leq T}\|u(t)\|_{s}^{2}+\frac{1}{R}\left\|J^{s+\frac{1}{2}} u\right\|_{T}^{2}\right) .
\end{aligned}
$$

Admitting these propositions (see Section 5 and Section 6 for their proofs), let us go on and finish the proof of Theorem 3.1. In order to apply the above inequalities we have to regularize the $b_{k}, k=1,2$, by setting

$$
\varphi_{k, \mu, m}(x, \xi)=m^{n} \int_{\mathbb{R}^{n}} \chi(m(x-y)) \varphi_{k, \mu}(y, \xi) d y \quad \text { and } \quad b_{k, m}=\sum_{\mu} \alpha_{k, \mu} \varphi_{k, \mu, m}
$$

where $\chi$ is a non negative $C^{\infty}$ function with support in the unit ball and whose integral is equal to 1 . Note that $\varphi_{k, \mu, m}$ has its support (with respect to $x$ ) in a compact set which is slightly larger that $Q_{\mu}^{*}$ but this has no effect on the proofs. Since we can write

$$
\partial_{t} u=i \mathscr{L} u+T_{b_{1, m}} \cdot \nabla_{x} u+T_{b_{2, m}} \cdot \nabla_{x} \bar{u}+C_{1} u+C_{2} \bar{u}+f_{m},
$$

where $f_{m}=f+T_{b_{1}-b_{1, m}} . \nabla u+T_{b_{2}-b_{2, m}} . \nabla \bar{u}$, we can apply Proposition 3.1 to obtain

$$
\begin{aligned}
\sup _{[0, T]}\left\|\mathbf{C}_{m} u\right\|_{s}^{2} \leq\left\|\mathbf{C}_{m} u_{0}\right\|_{s}^{2} & +2 \int_{0}^{T}\left|\left\langle\mathbf{C}_{m} J^{s} f_{m}, \mathbf{C}_{m} J^{s} u\right\rangle\right| d t \\
& +A \sup _{k, \mu} \nu_{N}\left(\varphi_{k, \mu, m}\right)\left(R T \sup _{[0, T]}\|u\|_{s}^{2}+\frac{1}{R}\left\|J^{s+\frac{1}{2}} u\right\|_{T}^{2}\right),
\end{aligned}
$$


where the operator $\mathbf{C}$ is denoted here by $\mathbf{C}_{m}$ to indicate its dependence on $m$. Now, clearly, we have $\nu_{N}\left(\varphi_{k, \mu, m}\right) \leq A m^{N^{2}} \sup _{1 \leq j \leq N} \sup _{|\beta| \leq N}\left\|\langle\xi\rangle^{|\beta|} \partial_{\xi}^{\beta} \varphi_{k, \mu}\right\|_{L^{\infty}}^{j} \leq A m^{N^{2}}$. Hence,

$$
\begin{aligned}
\sup _{[0, T]}\left\|\mathbf{C}_{m} u\right\|_{s}^{2} & \leq\left\|\mathbf{C}_{m} u_{0}\right\|_{s}^{2}+2 \int_{0}^{T}\left|\left\langle\mathbf{C}_{m} J^{s} f, \mathbf{C}_{m} J^{s} u\right\rangle\right| d t+2 \int_{0}^{T}\left|\left\langle\mathbf{C}_{m} J^{s} T_{b_{1}-b_{1, m}} \nabla u, \mathbf{C}_{m} J^{s} u\right\rangle\right| d t \\
& +2 \int_{0}^{T}\left|\left\langle\mathbf{C}_{m} J^{s} T_{b_{2}-b_{2, m}} \nabla \bar{u}, \mathbf{C}_{m} J^{s} u\right\rangle\right| d t+A m^{N^{2}}\left(R T \sup _{[0, T]}\|u\|_{s}^{2}+\frac{1}{R}\left\|J^{s+\frac{1}{2}} u\right\|_{T}^{2}\right),
\end{aligned}
$$

and the problem now is to estimate the third and fourth terms in the right hand side of this inequality. This is done in the following lemma.

Lemma 3.1 Let $\tilde{u}$ stand for $u$ or $\bar{u}$, and $\sigma=\inf \{\varrho, 1\}$. Then, there exists a constant $A$ such that, for all $k \in\{1,2\}, m \geq 1, R \geq 1$ and $m^{\prime} \geq m$,

$$
\begin{array}{r}
\int_{0}^{T}\left|\left\langle\mathbf{C}_{m} J^{s} T_{b_{k}-b_{k, m}} \nabla \tilde{u}, \mathbf{C}_{m} J^{s} u\right\rangle\right| d t \leq\left(\frac{A m^{2 N^{2}}}{m^{\prime \sigma}}+\frac{A m^{\prime 3 N^{2}}}{R}\right)\left\|J^{s+\frac{1}{2}} u\right\|_{T}^{2} \\
+A m^{\prime 3 N^{2}} T \sup _{[0, T]}\|u\|_{s}^{2}+\frac{A}{m^{\sigma}}\left\|J^{s+\frac{1}{2}} \mathbf{C}_{m} u\right\|_{T}^{2} .
\end{array}
$$

See Section 7 for the proof of this lemma. Applying this lemma yields

$$
\begin{gathered}
\sup _{[0, T]}\left\|\mathbf{C}_{m} u\right\|_{s}^{2} \leq\left\|\mathbf{C}_{m} u_{0}\right\|_{s}^{2}+2 \int_{0}^{T}\left|\left\langle\mathbf{C}_{m} J^{s} f, \mathbf{C}_{m} J^{s} u\right\rangle\right| d t+\frac{A}{m^{\sigma}}\left\|J^{s+\frac{1}{2}} \mathbf{C}_{m} u\right\|_{T}^{2} \\
+\left(\frac{A m^{2 N^{2}}}{m^{\prime \sigma}}+\frac{A m^{\prime 3 N^{2}}}{R}\right)\left\|J^{s+\frac{1}{2}} u\right\|_{T}^{2}+A m^{\prime 3 N^{2}} R T \sup _{[0, T]}\|u\|_{s}^{2}
\end{gathered}
$$

an inequality that we can improve, thanks to Proposition 3.2, as follows

$$
\begin{array}{r}
\sup _{[0, T]}\left\|\mathbf{C}_{m} u\right\|_{s}^{2} \leq\left\|\mathbf{C}_{m} u_{0}\right\|_{s}^{2}+2 \int_{0}^{T}\left|\left\langle\mathbf{C}_{m} J^{s} f, \mathbf{C}_{m} J^{s} u\right\rangle\right| d t+\frac{A}{m^{\sigma}} \sum_{j=1}^{4} \sup _{\mu} \int_{0}^{T}\left|\left\langle\Psi_{j, \mu} \mathbf{C}_{m} J^{s} f, \mathbf{C}_{m} J^{s} u\right\rangle\right| d t \\
+\frac{A\left(1+T m^{N}\right)}{m^{\sigma}} \sup _{[0, T]}\left\|\mathbf{C}_{m} u\right\|_{s}^{2}+\left(\frac{A m^{2 N^{2}}}{m^{\prime \sigma}}+\frac{A m^{\prime 3 N^{2}}}{R}\right)\left\|J^{s+\frac{1}{2}} u\right\|_{T}^{2}+A m^{\prime 3 N^{2}} R T \sup _{[0, T]}\|u\|_{s}^{2} \\
\leq\left\|\mathbf{C}_{m} u_{0}\right\|_{s}^{2}+2 \int_{0}^{T}\left|\left\langle\mathbf{C}_{m} J^{s} f, \mathbf{C}_{m} J^{s} u\right\rangle\right| d t+\frac{A}{m^{\sigma}} \sum_{j=1}^{4} \sup _{\mu} \int_{0}^{T}\left|\left\langle\Psi_{j, \mu} \mathbf{C}_{m} J^{s} f, \mathbf{C}_{m} J^{s} u\right\rangle\right| d t \\
+\frac{A\left(1+T m^{N}\right)}{m^{\sigma}} \sup _{[0, T]}\left\|\mathbf{C}_{m} u\right\|_{s}^{2}+\left(\frac{A m^{2 N^{2}}}{m^{\prime \sigma}}+\frac{A m^{\prime 3 N^{2}}}{R}\right) \sum_{j=1}^{4} \sup _{\mu} \int_{0}^{T}\left|\left\langle\Psi_{j, \mu} J^{s} f, J^{s} u\right\rangle\right| d t \\
+\left(\frac{A m^{2 N^{2}}}{m^{\prime \sigma}}+\frac{A m^{\prime 3 N^{2}}}{R}\right)\left(1+T m^{N}\right) \sup _{[0, T]}\|u\|_{s}^{2}+A m^{\prime 3 N^{2}} R T \sup _{[0, T]}\|u\|_{s}^{2},
\end{array}
$$

where $\Psi_{j, \mu}=\psi_{j}(x-\mu, D)$. Next, by taking $m$ such that, for example, $m^{\sigma} \geq 4 A$ and $T$ such 
that $T m^{N} \leq 1$, we get

$$
\begin{array}{r}
\sup _{[0, T]}\left\|\mathbf{C}_{m} u\right\|_{s}^{2} \leq 2\left\|\mathbf{C}_{m} u_{0}\right\|_{s}^{2}+4 \int_{0}^{T}\left|\left\langle\mathbf{C}_{m} J^{s} f, \mathbf{C}_{m} J^{s} u\right\rangle\right| d t+\sum_{j=1}^{4} \sup _{\mu} \int_{0}^{T}\left|\left\langle\Psi_{j, \mu} \mathbf{C}_{m} J^{s} f, \mathbf{C}_{m} J^{s} u\right\rangle\right| d t \\
+\left(\frac{2 A m^{2 N^{2}}}{m^{\prime \sigma}}+\frac{2 A m^{\prime 3 N^{2}}}{R}\right) \sum_{j=1}^{4} \sup _{\mu} \int_{0}^{T}\left|\left\langle\Psi_{j, \mu} J^{s} f, J^{s} u\right\rangle\right| d t \\
+\left(\frac{A m^{2 N^{2}}}{m^{\prime \sigma}}+\frac{A m^{\prime 3 N^{2}}}{R}+A m^{\prime 3 N^{2}} R T\right) \sup _{[0, T]}\|u\|_{s}^{2},
\end{array}
$$

and by using the second part of Proposition 3.1, we obtain

$$
\begin{aligned}
\sup _{[0, T]}\|u\|_{s}^{2} & \leq A m^{2 N^{2}}\left(m^{2 N^{2}}\left\|u_{0}\right\|_{s}^{2}+\int_{0}^{T}\left|\left\langle\mathbf{C}_{m} J^{s} f, \mathbf{C}_{m} J^{s} u\right\rangle\right| d t+\sum_{j=1}^{4} \sup _{\mu} \int_{0}^{T}\left|\left\langle\Psi_{j, \mu} \mathbf{C}_{m} J^{s} f, \mathbf{C}_{m} J^{s} u\right\rangle\right| d t\right) \\
& +\left(\frac{A m^{4 N^{2}}}{m^{\prime \sigma}}+\frac{A m^{\prime 5 N^{2}}}{R}\right) \sum_{j=1}^{4} \sup _{\mu} \int_{0}^{T}\left|\left\langle\Psi_{j, \mu} J^{s} f, J^{s} u\right\rangle\right| d t+C\left(m, m^{\prime}, R, T\right) \sup _{[0, T]}\|u\|_{s}^{2},
\end{aligned}
$$

where $C\left(m, m^{\prime}, R, T\right)=\frac{A m^{4 N^{2}}}{m^{\prime \sigma}}+\frac{A m^{\prime 5 N^{2}}}{R}+A m^{\prime 5 N^{2}} R T+\frac{A m^{4 N^{2}}}{R^{2}}$. Finally, $m$ being fixed (and depending only on $A$ ), we take $m^{\prime}$ such that $\frac{A m^{4 N^{2}}}{m^{\prime \sigma}} \leq \frac{1}{8}$, then we take $R$ such that $\frac{A m^{\prime 5 N^{2}}}{R} \leq \frac{1}{8}$ and $\frac{A m^{4 N^{2}}}{R^{2}} \leq \frac{1}{8}$, and last we take $T$ such that $A m^{\prime 5 N^{2}} R T \leq \frac{1}{8}$. With these choices, we have of course $C\left(m, m^{\prime}, R, T\right) \leq \frac{1}{2}$ which allows to bound $\sup _{[0, T]}\|u\|_{s}^{2}$ and to get (10) (and also (11), thanks to Proposition 3.2) with

$$
I_{T}(v, w)=\int_{0}^{T}\left|\left\langle\mathbf{C}^{\star} \mathbf{C} v, w\right\rangle\right| d t+\sum_{j=1}^{4} \sup _{\mu} \int_{0}^{T}\left|\left\langle\mathbf{C}^{\star} \Psi_{j, \mu} \mathbf{C} v, w\right\rangle\right| d t+\sup _{\mu} \int_{0}^{T}\left|\left\langle\Psi_{j, \mu} v, w\right\rangle\right| d t .
$$

In fact, we have proved (10) and (11) only for $T=T_{0}$ and $T_{0}$ is sufficiently small. Let us show, if $T_{0}<T$, that they hold true in the whole interval $[0, T]$ where the solution $u$ is defined. Indeed, note first that the $T_{0}$ as determined above depends only on the constant $A$ (so, only on $n, s, \varrho, A_{1}$ and $A_{2}$ ) but not on the given function (or distribution) $f$. Next, take a $T_{1} \leq T_{0}$ such that $T_{1}=T / n_{1}$, with some integer $n_{1} \geq 2$. Then, if we consider the function $v(x, t)=u\left(x, t+T_{1}\right)$, we note that $v$ is a solution (defined at least in $\left[0, T-T_{1}\right]$ ) of (8) with $v(0)=u\left(T_{1}\right)$ and $g(x, t)=f\left(x, t+T_{1}\right)$ instead of $f(x, t)$. It follows from the above arguments that $v$ satisfies (10) and (11) for $T=T_{0}$ and hence for $T=T_{1}$. Since

$$
\begin{aligned}
& \sup _{\left[T_{1}, 2 T_{1}\right]}\|u\|_{s}^{2}=\sup _{\left[0, T_{1}\right]}\|v\|_{s}^{2} \leq A\left(\left\|u\left(T_{1}\right)\right\|_{s}^{2}+I_{T_{1}}\left(J^{s} g, J^{s} v\right)\right) \leq A\left(\left\|u\left(T_{1}\right)\right\|_{s}^{2}+I_{2 T_{1}}\left(J^{s} f, J^{s} u\right)\right) \\
& \leq A\left(A\left\|u_{0}\right\|_{s}^{2}+A I_{T_{1}}\left(J^{s} f, J^{s} u\right)+I_{2 T_{1}}\left(J^{s} f, J^{s} u\right)\right) \leq\left(A^{2}+A\right)\left(\left\|u_{0}\right\|_{s}^{2}+I_{2 T_{1}}\left(J^{s} f, J^{s} u\right)\right),
\end{aligned}
$$

we obtain that $u$ satisfies (10) and (11) for $T=2 T_{1}$ and with the constant $A^{2}+A$ instead of $A$. Repeating this argument, we obtain that $u$ satisfies (10) and (11) on $\left[0, n_{1} T_{1}\right]=[0, T]$ and with the constant $\sum_{j=1}^{n_{1}} A^{j} \simeq A^{T / T_{1}}$ instead of $A$. 
As for the existence, let us consider the following approximating Cauchy problem :

$$
\left\{\begin{array}{l}
\partial_{t} u=i \mathscr{L} u+T_{b_{1}} \nabla h(\varepsilon D) u+T_{b_{2}} \nabla h(\varepsilon D) \bar{u}+C_{1} u+C_{2} \bar{u}+f(x, t) \\
u(x, 0)=u_{0} \in H^{s}\left(\mathbb{R}^{n}\right)
\end{array}\right.
$$

where $h$ is a non negative $C^{\infty}$ function on $\mathbb{R}^{n}$ which is equal to 1 near 0 and has a compact support. It is easy to see, if $T$ is such that $\sup _{[0, T]}\|f\|_{s}<+\infty$, that the above problem has a unique solution, denoted by $u_{\varepsilon}$, which is in $C\left([0, T] ; H^{s}\left(\mathbb{R}^{n}\right)\right)$. Indeed, the Cauchy problem (12) is clearly equivalent to the integral equation

$$
u=e^{i t \mathscr{L}} u_{0}+\int_{0}^{t} e^{i\left(t-t^{\prime}\right) \mathscr{L}}\left(T_{b_{1}} \nabla h(\varepsilon D) u+T_{b_{2}} \nabla h(\varepsilon D) \bar{u}+C_{1} u+C_{2} \bar{u}+f\left(x, t^{\prime}\right)\right) d t^{\prime}
$$

and one can easily show that the map defined by the right handside of this equation is a contraction in $C\left(\left[0, T_{\varepsilon}\right] ; H^{s}\left(\mathbb{R}^{n}\right)\right)$ with some $T_{\varepsilon}>0$ sufficiently small, which allows one to apply the fixed point theorem and to get a solution $u_{\varepsilon}$. Now, since $T_{\varepsilon}$ does not depend on the data $u_{0}$ and $f$, one can extend $u_{\varepsilon}$ to a solution of (12) on the whole interval $[0, T]$.

The idea now is to let $\varepsilon$ tend to 0 . This is possible because $u_{\varepsilon}$ satisfies the estimates (10) and (11) and even uniformly with respect to $\varepsilon$. Indeed, it is sufficient to remark that the Cauchy problem (12) is of the same type as (8) because we can write

$$
T_{b_{k}} \nabla h(\varepsilon D)=T_{b_{k, \varepsilon}} \nabla
$$

where $b_{k, \varepsilon}(x, \xi)=b_{k}(x, \xi) h(\varepsilon \xi)$ and $b_{k, \varepsilon}$ satisfies the assumptions of Theorem 3.1 uniformly in $\varepsilon$. Hence, we have in particular

$$
\sup _{[0, T]}\left\|u_{\varepsilon}\right\|_{s}^{2} \leq A\left\|u_{0}\right\|_{s}^{2}+A I_{T}\left(J^{s} f, J^{s} u_{\varepsilon}\right),
$$

and it follows from Calderon-Vaillancourt theorem that

$$
A I_{T}\left(J^{s} f, J^{s} u_{\varepsilon}\right) \leq A A^{\prime} \sup _{[0, T]}\left\|u_{\varepsilon}\right\|_{s} \int_{0}^{T}\|f\|_{s} d t \leq \frac{1}{2} \sup _{[0, T]}\left\|u_{\varepsilon}\right\|_{s}^{2}+\frac{1}{2}\left(A A^{\prime}\right)^{2}\left(\int_{0}^{T}\|f\|_{s} d t\right)^{2},
$$

so that,

$$
\sup _{[0, T]}\left\|u_{\varepsilon}\right\|_{s} \leq A\left\|u_{0}\right\|_{s}+A \int_{0}^{T}\|f\|_{s} d t
$$

Next, to check the convergence of $u_{\varepsilon}$, let us consider $v=u_{\varepsilon}-u_{\varepsilon^{\prime}}$. It is clear that $v$ is the solution of (12) with $u_{0}=0$ and

$$
f=T_{b_{1}} \nabla\left(h(\varepsilon D)-h\left(\varepsilon^{\prime} D\right)\right) u_{\varepsilon^{\prime}}+T_{b_{2}} \nabla\left(h(\varepsilon D)-h\left(\varepsilon^{\prime} D\right)\right) \bar{u}_{\varepsilon^{\prime}} .
$$

Therefore, it follows from (13) that

$$
\sup _{[0, T]}\|v\|_{s} \leq A \int_{0}^{T}\left\|T_{b_{1}} \nabla\left(h(\varepsilon D)-h\left(\varepsilon^{\prime} D\right)\right) u_{\varepsilon^{\prime}}+T_{b_{2}} \nabla\left(h(\varepsilon D)-h\left(\varepsilon^{\prime} D\right)\right) \bar{u}_{\varepsilon^{\prime}}\right\|_{s} d t,
$$


and from the boundedness of the $T_{b_{k}}$ in the Sobolev spaces that

$$
\sup _{[0, T]}\|v\|_{s} \leq A\left|\varepsilon-\varepsilon^{\prime}\right| \int_{0}^{T}\left\|u_{\varepsilon^{\prime}}\right\|_{s+2} d t \leq A\left|\varepsilon-\varepsilon^{\prime}\right| T \sup _{[0, T]}\left\|u_{\varepsilon^{\prime}}\right\|_{s+2},
$$

that is, thanks to (13),

$$
\sup _{[0, T]}\left\|u_{\varepsilon}-u_{\varepsilon^{\prime}}\right\|_{s} \leq A\left|\varepsilon-\varepsilon^{\prime}\right|\left(\left\|u_{0}\right\|_{s+2}+\int_{0}^{T}\|f\|_{s+2} d t\right),
$$

which proves that $\left(u_{\varepsilon}\right)$ is a Cauchy sequence in $C\left([0, T] ; H^{s}\left(\mathbb{R}^{n}\right)\right)$ if one assumes that $u_{0} \in$ $H^{s+2}\left(\mathbb{R}^{n}\right)$ and $f \in L^{1}\left([0, T] ; H^{s+2}\left(\mathbb{R}^{n}\right)\right)$. In this case, $u_{\varepsilon} \rightarrow u$ in $C\left([0, T] ; H^{s}\left(\mathbb{R}^{n}\right)\right)$ when $\varepsilon \rightarrow 0$, and by passing to the limit in (12), we obtain that $u$ is a solution of (8). Moreover, by passing to the limit in (13), we get

$$
\sup _{[0, T]}\|u\|_{s} \leq A\left(\left\|u_{0}\right\|_{s}+\int_{0}^{T}\|f\|_{s} d t\right) .
$$

Now, if we have only $u_{0} \in H^{s}\left(\mathbb{R}^{n}\right)$ and $f \in L^{1}\left([0, T] ; H^{s}\left(\mathbb{R}^{n}\right)\right)$, by density of the smooth functions, we can take sequences $\left(u_{0}^{j}\right)$ in $H^{s+2}\left(\mathbb{R}^{n}\right)$ and $\left(f^{j}\right)$ in $L^{1}\left([0, T] ; H^{s+2}\left(\mathbb{R}^{n}\right)\right)$ such that $\left\|u_{0}^{j}-u_{0}\right\|_{s} \rightarrow 0$ and $\int_{0}^{T}\left\|f^{j}-f\right\|_{s} d t \rightarrow 0$, and we can consider the solution $u^{j}$ of (8) associated to the data $u_{0}^{j}$ and $f^{j}$. Then, $u^{j}-u^{k}$ is the solution of (8) associated to the data $u_{0}^{j}-u_{0}^{k}$ and $f^{j}-f^{k}$. Hence, thanks to (17),

$$
\sup _{[0, T]}\left\|u^{j}-u^{k}\right\|_{s} \leq A\left(\left\|u_{0}^{j}-u_{0}^{k}\right\|_{s}+\int_{0}^{T}\left\|f^{j}-f^{k}\right\|_{s} d t\right),
$$

which shows that $\left(u^{j}\right)$ is a Cauchy sequence in $C\left([0, T] ; H^{s}\left(\mathbb{R}^{n}\right)\right)$ which is then convergent to some $u \in C\left([0, T] ; H^{s}\left(\mathbb{R}^{n}\right)\right)$. Of course, $u$ is a solution of (8) and satisfies the estimates (10), (11) and also (17). This achieves the proof of Theorem (3.1).

\section{The nonlinear equation}

Consider the nonlinear Cauchy problem :

$$
\left\{\begin{array}{l}
\partial_{t} u=i \mathscr{L} u+F\left(u, \nabla_{x} u, \bar{u}, \nabla_{x} \bar{u}\right), \quad t \in \mathbb{R}, x \in \mathbb{R}^{n}, \\
u(x, 0)=u_{0}(x) \in H^{s}\left(\mathbb{R}^{n}\right),
\end{array}\right.
$$

where the function $F(u, v, \bar{u}, \bar{v})$ is sufficiently regular in $\mathbb{C} \times \mathbb{C}^{n} \times \mathbb{C} \times \mathbb{C}^{n}$ and vanishes to the third order at 0 , the operator $\mathscr{L}$ has the form

$$
\mathscr{L}=\sum_{j \leq k} \partial_{x_{j}}^{2}-\sum_{j>k} \partial_{x_{j}}^{2}
$$

with a fixed $k \in\{1,2, \ldots, n\}, H^{s}\left(\mathbb{R}^{n}\right)$ is the usual Sobolev space on $\mathbb{R}^{n}$, and $s=\frac{n}{2}+2+\varrho$, $\varrho>0$. Using Bony's linearization formula, (18) is equivalent to

$$
\left\{\begin{array}{l}
\partial_{t} u=i \mathscr{L} u+T_{b_{1}} \nabla_{x} u+T_{b_{2}} \nabla_{x} \bar{u}+T_{a_{1}} u+T_{a_{2}} \bar{u}+R\left(u, \nabla_{x} u, \bar{u}, \nabla_{x} \bar{u}\right) \\
u(x, 0)=u_{0}(x) \in H^{s}\left(\mathbb{R}^{n}\right)
\end{array}\right.
$$


where $R\left(u, \nabla_{x} u, \bar{u}, \nabla_{x} \bar{u}\right)$ is Bony's remainder and

$$
\begin{array}{rlrl}
b_{1} & =\partial_{v} F\left(u, \nabla_{x} u, \bar{u}, \nabla_{x} \bar{u}\right), & b_{2} & =\partial_{\bar{v}} F\left(u, \nabla_{x} u, \bar{u}, \nabla_{x} \bar{u}\right), \\
a_{1}=\partial_{u} F\left(u, \nabla_{x} u, \bar{u}, \nabla_{x} \bar{u}\right), & a_{2}=\partial_{\bar{u}} F\left(u, \nabla_{x} u, \bar{u}, \nabla_{x} \bar{u}\right) .
\end{array}
$$

Recall that $R\left(u, \nabla_{x} u, \bar{u}, \nabla_{x} \bar{u}\right) \in H^{2(s-1)-\frac{n}{2}}\left(\mathbb{R}^{n}\right)$ if $u \in H^{s}\left(\mathbb{R}^{n}\right), s>\frac{n}{2}+1$. Note also that it follows from Proposition 2.1 that the $b_{j}$ and $a_{j}, j=1$ or 2 , are in $H^{s-1}\left(\mathbb{R}^{n}\right)$ if $u \in H^{s}\left(\mathbb{R}^{n}\right)$, $s>\frac{n}{2}+1$, and that

$$
\left\|b_{j}\right\|_{s-1} \leq C\left(\|u\|_{L^{\infty}},\|\nabla u\|_{L^{\infty}}\right)\|u\|_{s}, \quad\left\|a_{j}\right\|_{s-1} \leq C\left(\|u\|_{L^{\infty}},\|\nabla u\|_{L^{\infty}}\right)\|u\|_{s}, \quad j=1,2 .
$$

Moreover, by introducing the notations

$$
\begin{array}{ll}
b_{1}^{0}=\partial_{v} F\left(u_{0}, \nabla_{x} u_{0}, \bar{u}_{0}, \nabla_{x} \bar{u}_{0}\right), & b_{2}^{0}=\partial_{\bar{v}} F\left(u_{0}, \nabla_{x} u_{0}, \bar{u}_{0}, \nabla_{x} \bar{u}_{0}\right), \\
a_{1}^{0}=\partial_{u} F\left(u_{0}, \nabla_{x} u_{0}, \bar{u}_{0}, \nabla_{x} \bar{u}_{0}\right), & a_{2}^{0}=\partial_{\bar{u}} F\left(u_{0}, \nabla_{x} u_{0}, \bar{u}_{0}, \nabla_{x} \bar{u}_{0}\right),
\end{array}
$$

the above Cauchy problem is in fact equivalent to

$$
\left\{\begin{array}{l}
\partial_{t} u=i \mathscr{L} u+T_{b_{1}^{0}} \nabla_{x} u+T_{b_{2}^{0}} \nabla_{x} \bar{u}+T_{a_{1}^{0}} u+T_{a_{2}^{0}} \bar{u}+\widetilde{R}\left(u, \nabla_{x} u, \bar{u}, \nabla_{x} \bar{u}\right) \\
u(x, 0)=u_{0}(x) \in H^{s}\left(\mathbb{R}^{n}\right)
\end{array}\right.
$$

where

$$
\widetilde{R}\left(u, \nabla_{x} u, \bar{u}, \nabla_{x} \bar{u}\right)=T_{b_{1}-b_{1}^{0}} \nabla_{x} u+T_{b_{2}-b_{2}^{0}} \nabla_{x} \bar{u}+T_{a_{1}-a_{1}^{0}} u+T_{a_{2}-a_{2}^{0}} \bar{u}+R\left(u, \nabla_{x} u, \bar{u}, \nabla_{x} \bar{u}\right)
$$

Clearly, the last Cauchy problem is of the same type as (8) which is studied in Theorem 3.1 and in fact we are going to apply that theorem to

$$
\left\{\begin{array}{l}
\partial_{t} u=i \mathscr{L} u+T_{b_{1}^{0}} \nabla_{x} u+T_{b_{2}^{0}} \nabla_{x} \bar{u}+T_{a_{1}^{0}} u+T_{a_{2}^{0}} \bar{u}+f \\
u(x, 0)=u_{0}(x) \in H^{s}\left(\mathbb{R}^{n}\right) .
\end{array}\right.
$$

This is possible because $b_{1}^{0}$ and $b_{2}^{0}$ satisfy the assumptions of Theorem 3.1. Indeed, it follows from the Taylor formula and the assumptions on $F$ that one can write for example

$$
b_{1}^{0}=\partial_{v} F\left(z_{0}\right)=u_{0} G_{1}\left(z_{0}\right)+\nabla_{x} u_{0} G_{2}\left(z_{0}\right)+\bar{u}_{0} G_{3}\left(z_{0}\right)+\nabla_{x} \bar{u}_{0} G_{4}\left(z_{0}\right)
$$

where $z_{0}=\left(u_{0}, \nabla_{x} u_{0}, \bar{u}_{0}, \nabla_{x} \bar{u}_{0}\right)$ and $G_{1}, G_{2}, G_{3}$ and $G_{4}$ are sufficiently regular and vanish at 0 . Since $s-1>\frac{n}{2}$, we know that the $G_{i}\left(z_{0}\right)$ are in $H^{s-1}\left(\mathbb{R}^{n}\right)$ and it follows from (23) and Lemma 2.2 that $b_{1}^{0}$ satisfies the assumption (9) of Theorem 3.1, that is, one can write

$$
b_{1}^{0}=\sum_{\mu} \alpha_{1, \mu} \varphi_{1, \mu}
$$

where $\alpha_{1, \mu}=\left\|q_{\mu} b_{1}^{0}\right\|_{H^{s-1}}, \varphi_{1, \mu}=q_{\mu} b_{1}^{0} / \alpha_{1, \mu}$, and $\sum_{\mu} q_{\mu}=1$ is a smooth partition of unity with $q_{\mu}(x)=q(x-\mu)$ and $\operatorname{supp}(q) \subset Q_{0}^{*}$. Note that we have precisely the bound

$$
\begin{gathered}
\sum_{\mu}\left\|q_{\mu} b_{1}^{0}\right\|_{H^{s-1}} \leq C\left(\left\|u_{0}\right\|_{H^{s-1}}\left\|G_{1}\left(z_{0}\right)\right\|_{H^{s-1}}+\left\|\nabla_{x} u_{0}\right\|_{H^{s-1}}\left\|G_{2}\left(z_{0}\right)\right\|_{H^{s-1}}\right. \\
\left.+\left\|\bar{u}_{0}\right\|_{H^{s-1}}\left\|G_{3}\left(z_{0}\right)\right\|_{H^{s-1}}+\left\|\nabla_{x} \bar{u}_{0}\right\|_{H^{s-1}}\left\|G_{4}\left(z_{0}\right)\right\|_{H^{s-1}}\right)
\end{gathered}
$$


with some positive constant $C$. Of course, the same is true for $b_{2}^{0}$. Moreover, since $a_{1}^{0}$ and $a_{2}^{0}$ are bounded (they are in $H^{s-1}\left(\mathbb{R}^{n}\right)$ ), the paramultiplication operators $T_{a_{1}^{0}}$ and $T_{a_{2}^{0}}$ are bounded in $H^{s}\left(\mathbb{R}^{n}\right)$.

Now, by application of Theorem 3.1 to (22), let us consider the unique solution of (22) with $f=0$ and denote it by $U(t) u_{0}$.

Next, for $T>0$, let us define the norms $\lambda_{1}(w), \lambda_{2}(w), \lambda_{3}(w)$ and $\lambda(w)$ by

$$
\begin{gathered}
\lambda_{1}(w)=\sup _{[0, T]}\|w\|_{s}, \quad \lambda_{2}(w)=\left\|J^{s+\frac{1}{2}} w\right\|_{T}, \quad \lambda_{3}(w)=\sup _{[0, T]}\left\|\partial_{t} w\right\|_{s-2}, \\
\lambda(w)=\max _{1 \leq i \leq 3} \lambda_{i}(w),
\end{gathered}
$$

the space $Z$ by

$$
Z=\left\{w \in C\left([0, T] ; H^{s}\left(\mathbb{R}^{n}\right)\right): w(x, 0)=u_{0}(x) \text { and } \lambda(w) \leq K\right\}
$$

where the positive constant $K$ is to be determined later, and, for $w \in C\left([0, T] ; H^{s}\left(\mathbb{R}^{n}\right)\right)$, the operator $\Upsilon$ by

$$
\Upsilon w(t)=U(t) u_{0}+\int_{0}^{t} U\left(t-t^{\prime}\right) \widetilde{R}\left(w\left(t^{\prime}\right), \nabla_{x} w\left(t^{\prime}\right), \bar{w}\left(t^{\prime}\right), \nabla_{x} \bar{w}\left(t^{\prime}\right)\right) d t^{\prime} .
$$

Let us first remark that $\Upsilon w$ satisfies

$$
\left\{\begin{array}{l}
\partial_{t} \Upsilon w=i \mathscr{L} \Upsilon w+T_{b_{1}^{0}} \nabla_{x} \Upsilon w+T_{b_{2}^{0}} \nabla_{x} \overline{\Upsilon w}+T_{a_{1}^{0}} \Upsilon w+T_{a_{2}^{0}} \overline{\Upsilon w}+\widetilde{R}\left(w, \nabla_{x} w, \bar{w}, \nabla_{x} \bar{w}\right) \\
\Upsilon w(0)=u_{0}
\end{array}\right.
$$

and that a fixed point of $\Upsilon$ will be a solution of (20), hence, a solution of (18). So, in what follows, we are going to study $\lambda(\Upsilon w)$ in order to prove that $\Upsilon$ has a fixed point in the complete metric space $(Z, \lambda)$. Let us also note that since the life time $T$ will be small, we can assume from now on that $T \leq 1$.

We start by applying Theorem 3.1 to (24). It follows from (10) and (11) that

$$
\max \left\{\lambda_{1}(\Upsilon w)^{2}, \lambda_{2}(\Upsilon w)^{2}\right\} \leq A\left(\left\|u_{0}\right\|_{s}^{2}+I_{T}\left(J^{s} \widetilde{R}, J^{s} \Upsilon w\right)\right)
$$

where, for simplicity, $\widetilde{R}=\widetilde{R}\left(w, \nabla_{x} w, \bar{w}, \nabla_{x} \bar{w}\right)$ and $I_{T}(u, v)$ is a finite sum of terms of the form

$$
\sup _{\mu \in \mathbb{Z}^{n}} \int_{0}^{T}\left|\left\langle G_{\mu} u, v\right\rangle\right| d t
$$

where $G_{\mu} \in \mathrm{Op} S_{0,0}^{0}$ and the semi-norms of its symbol are uniformly bounded with respect to $\mu$. Recall that the constant $A$ depends only on $n, s$ and $u_{0}$ and we remark right now a fact that will be useful later : if we let $u_{0}$ vary in a bounded subset of $H^{s}\left(\mathbb{R}^{n}\right)$, it follows from the linear theory that we can take the constant $A$ in the above inequality that depends only on that bounded set. The same remark holds for $\sup _{\mu}\left\|G_{\mu}\right\|_{\mathcal{L}\left(L^{2}\right)}$ or the semi-norms of the operators $G_{\mu}$ uniformly in $\mu$. 
Thus, we have to estimate uniformly in $\mu$ the following sum

$$
\begin{aligned}
& \int_{0}^{T}\left|\left\langle G_{\mu} J^{s} T_{b_{1}-b_{1}^{0}} \nabla_{x} w, J^{s} \Upsilon w\right\rangle\right| d t+\int_{0}^{T}\left|\left\langle G_{\mu} J^{s} T_{b_{2}-b_{2}^{0}} \nabla_{x} \bar{w}, J^{s} \Upsilon w\right\rangle\right| d t \\
& +\int_{0}^{T}\left|\left\langle G_{\mu} J^{s} T_{a_{1}-a_{1}^{0}} w, J^{s} \Upsilon w\right\rangle\right| d t+\int_{0}^{T}\left|\left\langle G_{\mu} J^{s} T_{a_{2}-a_{2}^{0}} \bar{w}, J^{s} \Upsilon w\right\rangle\right| d t \\
& +\int_{0}^{T}\left|\left\langle G_{\mu} J^{s} R\left(w, \nabla_{x} w, \bar{w}, \nabla_{x} \bar{w}\right), J^{s} \Upsilon w\right\rangle\right| d t
\end{aligned}
$$

First, let us consider the third term. It follows from the preceding remark, Cauchy-Schwarz inequality, Calderon-Vaillancourt theorem and Theorem 2.3 that

$$
\int_{0}^{T}\left|\left\langle G_{\mu} J^{s} T_{a_{1}-a_{1}^{0}} w, J^{s} \Upsilon w\right\rangle\right| d t \leq A\left\|a_{1}-a_{1}^{0}\right\|_{L^{\infty}} \int_{0}^{T}\|w\|_{s}\|\Upsilon w\|_{s} d t
$$

and from Proposition 2.1 that

$$
\left\|a_{1}-a_{1}^{0}\right\|_{L^{\infty}} \leq C\left(\|w\|_{s}\right)\|w\|_{s}+C\left(\left\|u_{0}\right\|_{s}\right)\left\|u_{0}\right\|_{s} \leq C(K) K+C\left(\left\|u_{0}\right\|_{s}\right)\left\|u_{0}\right\|_{s} \leq 2 C(K) K .
$$

Hence,

$$
\int_{0}^{T}\left|\left\langle G_{\mu} J^{s} T_{a_{1}-a_{1}^{0}} w, J^{s} \Upsilon w\right\rangle\right| d t \leq A T C(K) \lambda_{1}(w) \lambda_{1}(\Upsilon w) \leq A T C(K) \lambda(w) \lambda(\Upsilon w),
$$

with a modified constant $C(K)$.

The fourth term of $(26)$ is treated in the same manner.

Now, let us estimate the first term of (26). Using a smooth partition of unity $1=$ $\sum_{\nu \in \mathbb{Z}^{n}} \chi_{\nu}$, with $\chi_{\nu}(x)=\chi(x-\nu)$ and $\chi$ has a compact support, we can write

$$
\begin{gathered}
\left\langle G_{\mu} J^{s} T_{b_{1}-b_{1}^{0}} \nabla_{x} w, J^{s} \Upsilon w\right\rangle=\sum_{\nu}\left\langle J^{-\frac{1}{2}} G_{\mu} J^{s} T_{\chi_{\nu}\left(b_{1}-b_{1}^{0}\right)} \nabla_{x} w, J^{s+\frac{1}{2}} \Upsilon w\right\rangle \\
=\sum_{\nu}\left\langle G_{\mu, \nu}\langle x-\nu\rangle^{\sigma_{0}} T_{\chi_{\nu}\left(b_{1}-b_{1}^{0}\right)}\langle x-\nu\rangle^{\sigma_{0}} H_{\mu}\langle x-\nu\rangle^{-\sigma_{0}} J^{s+\frac{1}{2}} w,\langle x-\nu\rangle^{-\sigma_{0}} J^{s+\frac{1}{2}} \Upsilon w\right\rangle
\end{gathered}
$$

where $G_{\mu, \nu}=\langle x-\nu\rangle^{\sigma_{0}} J^{-\frac{1}{2}} G_{\mu} J^{s}\langle x-\nu\rangle^{-\sigma_{0}}$ and $H_{\nu}=\langle x-\nu\rangle^{-\sigma_{0}} J^{-s-\frac{1}{2}} \nabla\langle x-\nu\rangle^{\sigma_{0}}$. Next, it follows from the pseudodifferential composition formula and from Lemma 2.1 that $G_{\mu, \nu}$ is in $\mathrm{Op} S_{0,0}^{s-\frac{1}{2}}, H_{\nu}$ is in $\mathrm{Op} S_{1,0}^{\frac{1}{2}-s}$, and that their semi-norms are uniformly bounded with respect to $\mu$ and $\nu$. Going back to the first term of (26), these considerations in addition to Lemma 2.4 allow us to estimate it as follows :

$$
\begin{gathered}
\int_{0}^{T}\left|\left\langle G_{\mu} J^{s} T_{b_{1}-b_{1}^{0}} \nabla_{x} w, J^{s} \Upsilon w\right\rangle\right| d t \\
\leq \sum_{\nu} \int_{0}^{T}\left\|G_{\mu, \nu}\langle x-\nu\rangle^{\sigma_{0}} T_{\chi_{\nu}\left(b_{1}-b_{1}^{0}\right)}\langle x-\nu\rangle^{\sigma_{0}} H_{\nu}\right\|_{\mathcal{L}\left(L^{2}\right)}\left\|\frac{J^{s+\frac{1}{2}} w}{\langle x-\nu\rangle^{\sigma_{0}}}\right\|_{0}\left\|\frac{J^{s+\frac{1}{2}} \Upsilon w}{\langle x-\nu\rangle^{\sigma_{0}}}\right\|_{0} d t
\end{gathered}
$$




$$
\begin{aligned}
& \leq A \sum_{\nu} \int_{0}^{T}\left\|\langle x-\nu\rangle^{\sigma_{0}} T_{\chi_{\nu}\left(b_{1}-b_{1}^{0}\right)}\langle x-\nu\rangle^{\sigma_{0}}\right\|_{\mathcal{L}\left(H^{s-\frac{1}{2}}\right)}\left\|\frac{J^{s+\frac{1}{2}} w}{\langle x-\nu\rangle^{\sigma_{0}}}\right\|_{0}\left\|\frac{J^{s+\frac{1}{2}} \Upsilon w}{\langle x-\nu\rangle^{\sigma_{0}}}\right\|_{0} d t \\
& \leq A \sum_{\nu} \int_{0}^{T}\left\|\chi_{\nu}\left(b_{1}-b_{1}^{0}\right)\right\|_{L^{\infty}}\left\|\frac{J^{s+\frac{1}{2}} w}{\langle x-\nu\rangle^{\sigma_{0}}}\right\|_{0}\left\|\frac{J^{s+\frac{1}{2}} \Upsilon w}{\langle x-\nu\rangle^{\sigma_{0}}}\right\|_{0} d t \\
& \leq A \sum_{\nu} \sup _{[0, T]}\left\|\chi_{\nu}\left(b_{1}-b_{1}^{0}\right)\right\|_{L^{\infty}}\left\|J^{s+\frac{1}{2}} w\right\|_{T}\left\|J^{s+\frac{1}{2}} \Upsilon w\right\|_{T} .
\end{aligned}
$$

Now, it follows from the Taylor formula that we can write

$$
\begin{aligned}
b_{1}-b_{1}^{0}=\partial_{v} F(z)-\partial_{v} F\left(z_{0}\right) & =\left(w-u_{0}\right) G_{1}\left(z_{0}, z\right)+\nabla_{x}\left(w-u_{0}\right) G_{2}\left(z_{0}, z\right) \\
+ & \left(\bar{w}-\bar{u}_{0}\right) G_{3}\left(z_{0}, z\right)+\nabla_{x}\left(\bar{w}-\bar{u}_{0}\right) G_{4}\left(z_{0}, z\right)
\end{aligned}
$$

where, for simplicity, $z_{0}=\left(u_{0}, \nabla_{x} u_{0}, \bar{u}_{0}, \nabla_{x} \bar{u}_{0}\right)$ and $z=\left(w, \nabla_{x} w, \bar{w}, \nabla_{x} \bar{w}\right)$, and the $G_{k}$ 's are functions of the form

$$
\int_{0}^{1} F_{k}\left(z_{0}+\tau\left(z-z_{0}\right)\right) d \tau,
$$

$F_{k}$ being a second order partial derivative of $F$. Next, it follows from the assumption on $F$ that $G_{k}(0,0)=0$ for all $k$, from which one deduces easily that

$$
\left\|\chi_{\nu}\left(b_{1}-b_{1}^{0}\right)\right\|_{L^{\infty}} \leq C\left(\left\|\left(z_{0}, z\right)\right\|_{L^{\infty}}\right)\left\|\chi_{\nu}\left(z_{0}, z\right)\right\|_{L^{\infty}}\left\|\widetilde{\chi}_{\nu}\left(z_{0}, z\right)\right\|_{L^{\infty}},
$$

where $\tilde{\chi}_{\nu}$ is similar to $\chi_{\nu}$, and, by using the Sobolev injection, that is, Proposition $7.3(i)$, that

$$
\begin{gathered}
\left\|\chi_{\nu}\left(b_{1}-b_{1}^{0}\right)\right\|_{L^{\infty}} \leq C\left(\left\|\left(z_{0}, z\right)\right\|_{L^{\infty}}\right)\left\|\chi_{\nu}\left(z_{0}, z\right)\right\|_{H^{\sigma}\left([0, T] ; H^{s^{\prime}}\right)}\left\|\widetilde{\chi}_{\nu}\left(z_{0}, z\right)\right\|_{H^{\sigma}\left([0, T] ; H^{s^{\prime}}\right)} \\
\leq C(K)\left\|\chi_{\nu}\left(u_{0}, w\right)\right\|_{H^{\sigma}\left([0, T] ; H^{s^{\prime}+1}\right)}\left\|\widetilde{\chi}_{\nu}\left(u_{0}, w\right)\right\|_{H^{\sigma}\left([0, T] ; H^{s^{\prime}+1}\right)},
\end{gathered}
$$

where $\sigma>\frac{1}{2}$ and $s^{\prime}>\frac{n}{2}$. Thus, to obtain the summability in $\nu$ of $\left\|\chi_{\nu}\left(b_{1}-b_{1}^{0}\right)\right\|_{L^{\infty}}$, it is sufficient to prove that $\left\|\chi_{\nu}\left(u_{0}, w\right)\right\|_{H^{\sigma}\left([0, T] ; H^{s^{\prime}+1}\right)}$ is square summable in $\nu$. To this end and to get an explicit bound for the sum, let us apply the interpolation inequality of Proposition 7.3. This yields, by taking $\frac{1}{2}<\sigma<1$,

$$
\begin{aligned}
& \left\|\chi_{\nu}\left(u_{0}, w\right)\right\|_{H^{\sigma}\left([0, T] ; H^{s^{\prime}+1}\right)} \leq A\left\|\chi_{\nu}\left(u_{0}, w\right)\right\|_{L^{2}\left([0, T] ; H^{s^{\prime}+2}\right)}^{1-\sigma}\left\|\chi_{\nu}\left(u_{0}, w\right)\right\|_{H^{1}\left([0, T] ; H^{s^{\prime \prime}}\right)}^{\sigma} \\
& \leq A\left(\left\|\chi_{\nu}\left(u_{0}, w\right)\right\|_{L^{2}\left([0, T] ; H^{s^{\prime}+2}\right)}+\left\|\chi_{\nu}\left(u_{0}, w\right)\right\|_{L^{2}\left([0, T] ; H^{s^{\prime}+2}\right)}^{1-\sigma}\left\|\chi_{\nu} \partial_{t} w\right\|_{L^{2}\left([0, T] ; H^{s^{\prime \prime}}\right)}^{\sigma}\right)
\end{aligned}
$$

where $s^{\prime \prime}$ is such that $(1-\sigma)\left(s^{\prime}+2\right)+\sigma s^{\prime \prime}=s^{\prime}+1$, that is, $s^{\prime \prime}=s^{\prime}+2-\frac{1}{\sigma}$. One can choose $s^{\prime}$ and $\sigma$ such that $s^{\prime \prime}=s-2$, that is, such that $s^{\prime}=s-4+\frac{1}{\sigma}$. In fact, if $\sigma=\frac{1}{2}+\epsilon$, then $s^{\prime}=\frac{n}{2}+\varrho-\frac{4 \epsilon}{1+2 \epsilon}$ which is larger that $\frac{n}{2}$ if $\epsilon$ is small enough. With such a choice, we also have $s^{\prime}+2<s$, so, the expressions $\left\|\chi_{\nu}\left(u_{0}, w\right)\right\|_{L^{2}\left([0, T] ; H^{s^{\prime}+2}\right)}$ and $\left\|\chi_{\nu} \partial_{t} w\right\|_{L^{2}\left([0, T] ; H^{s^{\prime \prime}}\right)}$ are both square summable in $\nu$, which shows that $\left\|\chi_{\nu}\left(u_{0}, w\right)\right\|_{H^{\sigma}\left([0, T] ; H^{s^{\prime}+1}\right)}$ is itself square summable in $\nu$ and that, by applying Hölder's inequality,

$$
\sum_{\nu}\left\|\chi_{\nu}\left(u_{0}, w\right)\right\|_{H^{\sigma}\left([0, T] ; H^{s^{\prime}+1}\right)}^{2} \leq A \sum_{\nu}\left\|\chi_{\nu}\left(u_{0}, w\right)\right\|_{L^{2}\left([0, T] ; H^{s}\right)}^{2}
$$




$$
\begin{aligned}
& +A\left(\sum_{\nu}\left\|\chi_{\nu}\left(u_{0}, w\right)\right\|_{L^{2}\left([0, T] ; H^{s}\right)}^{2}\right)^{1-\sigma}\left(\sum_{\nu}\left\|\chi_{\nu} \partial_{t} w\right\|_{L^{2}\left([0, T] ; H^{s-2}\right)}^{2}\right)^{\sigma} \\
& \leq A\left(T \lambda_{1}(w)^{2}+\left(T \lambda_{1}(w)^{2}\right)^{1-\sigma}\left(T \lambda_{3}(w)^{2}\right)^{\sigma}\right) \\
& \leq A T \lambda(w)^{2}
\end{aligned}
$$

where, of course, the constant $A$ changes from one inequality to the other. Consequently,

$$
\sum_{\nu}\left\|\chi_{\nu}\left(b_{1}-b_{1}^{0}\right)\right\|_{L^{\infty}} \leq A C(K) T \lambda(w)^{2}
$$

which allows us finally to bound the first term of (26) as follows :

$$
\begin{gathered}
\int_{0}^{T}\left|\left\langle G_{\mu} J^{s} T_{b_{1}-b_{1}^{0}} \nabla_{x} w, J^{s} \Upsilon w\right\rangle\right| d t \leq A C(K) T \lambda(w)^{2} \lambda_{2}(w) \lambda_{2}(\Upsilon w) \\
\leq A C(K) K^{2} T \lambda(w) \lambda(\Upsilon w)
\end{gathered}
$$

The second term of (26) is treated in the same manner.

Let us now consider the last term of (26). As above, let $z$ stand for $\left(w, \nabla_{x} w, \bar{w}, \nabla_{x} \bar{w}\right)$. As $z \in H^{s-1}\left(\mathbb{R}^{n}\right)=H^{\frac{n}{2}+1+\varrho}\left(\mathbb{R}^{n}\right)$, it follows from Bony's formula, that is, Theorem 2.5, that $R(z) \in H^{2(s-1)-\frac{n}{2}}\left(\mathbb{R}^{n}\right)=H^{s+\varrho}\left(\mathbb{R}^{n}\right)$ and that

$$
\|R(z)\|_{s+\varrho} \leq C(K)\|z\|_{s-1} \leq C(K)\|w\|_{s} .
$$

Hence,

$$
\begin{aligned}
& \int_{0}^{T}\left|\left\langle G_{\mu} J^{s} R(z), J^{s} \Upsilon w\right\rangle\right| d t \\
& \leq A \int_{0}^{T}\|R(z)\|_{s}\|\Upsilon w\|_{s} d t \leq A C(K) \int_{0}^{T}\|w\|_{s}\|\Upsilon w\|_{s} d t \\
& \leq A C(K) T \lambda_{1}(w) \lambda_{1}(\Upsilon w) \leq A C(K) T \lambda(w) \lambda(\Upsilon w) .
\end{aligned}
$$

Thus, we have bounded all the terms of (26), which leads to the estimate

$$
\max \left\{\lambda_{1}(\Upsilon w), \lambda_{2}(\Upsilon w)\right\} \leq A\left\|u_{0}\right\|_{s}+\sqrt{A C(K) T \lambda(w) \lambda(\Upsilon w)},
$$

where the constants $A$ and $C(K)$ have changed of course.

It remains to estimate $\lambda_{3}(\Upsilon w)$. Recall that $\Upsilon w$ satisfies the Cauchy problem (24). Hence, applying Theorem 2.3 yields

$$
\begin{aligned}
& \left\|\partial_{t} \Upsilon w\right\|_{s-2} \leq\|\Upsilon w\|_{s}+A\left(\left\|b_{1}^{0}\right\|_{L^{\infty}}+\left\|b_{2}^{0}\right\|_{L^{\infty}}\right)\|\Upsilon w\|_{s-1}+A\left(\left\|a_{1}^{0}\right\|_{L^{\infty}}+\left\|a_{2}^{0}\right\|_{L^{\infty}}\right)\|\Upsilon w\|_{s-2} \\
& +A\left(\left\|b_{1}-b_{1}^{0}\right\|_{L^{\infty}}+\left\|b_{2}-b_{2}^{0}\right\|_{L^{\infty}}\right)\|w\|_{s-1}+A\left(\left\|a_{1}-a_{1}^{0}\right\|_{L^{\infty}}+\left\|a_{2}-a_{2}^{0}\right\|_{L^{\infty}}\right)\|w\|_{s-2}+\|R(z)\|_{s-2} \\
& \leq A\|\Upsilon w\|_{s}+A\left(\left\|b_{1}-b_{1}^{0}\right\|_{L^{\infty}}+\left\|b_{2}-b_{2}^{0}\right\|_{L^{\infty}}+\left\|a_{1}-a_{1}^{0}\right\|_{L^{\infty}}+\left\|a_{2}-a_{2}^{0}\right\|_{L^{\infty}}\right)\|w\|_{s}+\|R(z)\|_{s-2} .
\end{aligned}
$$


Now, as before, it follows from Proposition 7.3 that

$$
\left\|b_{j}-b_{j}^{0}\right\|_{L^{\infty}} \leq A\left\|b_{j}-b_{j}^{0}\right\|_{H^{\sigma}\left([0, T] ; H^{s^{\prime}}\right)} \leq A\left\|b_{j}-b_{j}^{0}\right\|_{L^{2}\left([0, T] ; H^{s^{\prime}+1}\right)}^{1-\sigma}\left\|b_{j}-b_{j}^{0}\right\|_{H^{1}\left([0, T] ; H^{s^{\prime \prime}}\right)}^{\sigma},
$$

where $j=1,2, \sigma>\frac{1}{2}, s^{\prime}>\frac{n}{2}$ and $s^{\prime \prime}$ is such that $(1-\sigma)\left(s^{\prime}+1\right)+\sigma s^{\prime \prime}=s^{\prime}$. In fact, we can take $s^{\prime \prime}=s-3$ which corresponds to $s^{\prime}=s+\frac{1}{\sigma}-4=\frac{n}{2}+\varrho+\frac{1}{\sigma}-2$; so, $s^{\prime}<s-2$ and if $\sigma$ is close enough to $\frac{1}{2}$, then, $s^{\prime}>\frac{n}{2}$. Therefore, with such a choice, we have

$$
\left\|b_{j}-b_{j}^{0}\right\|_{L^{\infty}} \leq A\left\|b_{j}-b_{j}^{0}\right\|_{L^{2}\left([0, T] ; H^{s-1}\right)}+A\left\|b_{j}-b_{j}^{0}\right\|_{L^{2}\left([0, T] ; H^{s-1}\right)}^{1-\sigma}\left\|\partial_{t} b_{j}\right\|_{L^{2}\left([0, T] ; H^{s-3}\right)}^{\sigma} .
$$

Next, applying Proposition 2.1 yields

$$
\begin{gathered}
\left\|b_{j}-b_{j}^{0}\right\|_{L^{2}\left([0, T] ; H^{s-1}\right)}^{2}=\int_{0}^{T}\left\|b_{j}-b_{j}^{0}\right\|_{s-1}^{2} d t \leq \int_{0}^{T}\left(C\left(\|z\|_{L^{\infty}}\right)\|z\|_{s-1}+C\left(\left\|z_{0}\right\|_{L^{\infty}}\right)\left\|z_{0}\right\|_{s-1}\right)^{2} d t \\
\leq C(K)^{2} T \lambda_{1}(w)^{2},
\end{gathered}
$$

and

$$
\begin{gathered}
\left\|\partial_{t} b_{j}\right\|_{L^{2}\left([0, T] ; H^{s-3}\right)}^{2}=\int_{0}^{T}\left\|\left(\partial_{v} F\right)^{\prime}(z) \partial_{t} z\right\|_{s-3}^{2} d t \leq A \int_{0}^{T}\left\|\left(\partial_{v} F\right)^{\prime}(z)\right\|_{s-2}^{2}\left\|\partial_{t} z\right\|_{s-3}^{2} d t \\
\leq A \int_{0}^{T}\left\|\left(\partial_{v} F\right)^{\prime}(z)\right\|_{s-2}^{2}\left\|\partial_{t} w\right\|_{s-2}^{2} d t \leq A T C(K)^{2} \lambda_{3}(w)^{2},
\end{gathered}
$$

which imply that

$$
\left\|b_{j}-b_{j}^{0}\right\|_{L^{\infty}} \leq A C(K) \sqrt{T} \lambda_{1}(w)+A C(K) \sqrt{T} \lambda_{1}(w)^{1-\sigma} \lambda_{3}(w)^{\sigma} \leq A C(K) \sqrt{T} \lambda(w) .
$$

Of course, the same inequality holds for $\left\|a_{j}-a_{j}^{0}\right\|_{L^{\infty}}, j=1,2$. Note that we have applied the following classical lemma :

Lemma 4.1 If $s>\frac{n}{2}$ and $|r| \leq s$, then, $H^{r}\left(\mathbb{R}^{n}\right) \cdot H^{s}\left(\mathbb{R}^{n}\right) \subset H^{r}\left(\mathbb{R}^{n}\right)$ with continuous injection.

Finally, it follows from Theorem 2.5 and Theorem 2.6 that

$$
\begin{gathered}
\|R(z)\|_{s-2}=\|R(z)\|_{\frac{n}{2}+\varrho} \leq\left\|R(z)-R\left(z_{0}\right)\right\|_{\frac{n}{2}+\varrho}+\left\|R\left(z_{0}\right)\right\|_{\frac{n}{2}+\varrho} \\
\leq C_{1}\left(\|z\|_{\frac{n+\varrho}{2}},\left\|z_{0}\right\|_{\frac{n+\varrho}{2}}\right)\left\|z-z_{0}\right\|_{\frac{n+\varrho}{2}}+C_{2}\left(\left\|z_{0}\right\|_{\frac{n+\varrho}{2}}\right)\left\|z_{0}\right\|_{\frac{n+\varrho}{2}} \\
\leq C_{1}\left(\|w\|_{\frac{n+\varrho}{2}+1},\left\|u_{0}\right\|_{\frac{n+\varrho}{2}+1}\right)\left\|w-u_{0}\right\|_{\frac{n+\varrho}{2}+1}+C_{2}\left(\left\|u_{0}\right\|_{\frac{n+\varrho}{2}+1}\right)\left\|u_{0}\right\|_{\frac{n+\varrho}{2}+1} \\
\leq C(K)\left\|w-u_{0}\right\|_{\frac{n+\varrho}{2}+1}+A\left\|u_{0}\right\|_{\frac{n+\varrho}{2}+1},
\end{gathered}
$$

and, using once again Proposition 7.3, we obtain

$$
\begin{gathered}
\sup _{[0, T]}\left\|w-u_{0}\right\|_{s^{\prime}} \leq A\left\|w-u_{0}\right\|_{H^{\sigma}\left([0, T] ; H^{s^{\prime}}\right)} \leq A\left\|w-u_{0}\right\|_{L^{2}\left([0, T] ; H^{s^{\prime}+1}\right)}^{1-\sigma}\left\|w-u_{0}\right\|_{H^{1}\left([0, T] ; H^{s^{\prime \prime}}\right)}^{\sigma} \\
\leq A\left\|w-u_{0}\right\|_{L^{2}\left([0, T] ; H^{s^{\prime}+1}\right)}+A\left\|w-u_{0}\right\|_{L^{2}\left([0, T] ; H^{s^{\prime}+1}\right)}^{1-\sigma}\left\|\partial_{t} w\right\|_{L^{2}\left([0, T] ; H^{s^{\prime \prime}}\right)}^{\sigma}
\end{gathered}
$$




$$
\begin{gathered}
\leq A\left\|w-u_{0}\right\|_{L^{2}\left([0, T] ; H^{s}\right)}+A\left\|w-u_{0}\right\|_{L^{2}\left([0, T] ; H^{s}\right)}^{1-\sigma}\left\|\partial_{t} w\right\|_{L^{2}\left([0, T] ; H^{s-2}\right)}^{\sigma} \\
\leq A \sqrt{T} \lambda_{1}(w)+A \sqrt{T} \lambda_{1}(w)^{1-\sigma} \lambda_{3}(w)^{\sigma} \leq A \sqrt{T} \lambda(w),
\end{gathered}
$$

where $s^{\prime}=\frac{n+\varrho}{2}+1<s, \sigma>\frac{1}{2}, s^{\prime \prime}=\frac{n+\varrho}{2}+2-\frac{1}{\sigma}$ and $s^{\prime \prime} \leq s-2$ if $\sigma$ is close to $\frac{1}{2}$. Hence,

$$
\sup _{[0, T]}\|R(z)\|_{s-2} \leq A\left\|u_{0}\right\|_{\frac{n+e}{2}+1}+A C(K) \sqrt{T} \lambda(w) .
$$

Thus, we have bounded all the terms of (32) and the result is that

$$
\begin{gathered}
\lambda_{3}(\Upsilon w) \leq A \lambda_{1}(\Upsilon w)+A C(K) \sqrt{T} \lambda(w) \lambda_{1}(w)+A\left\|u_{0}\right\|_{\frac{n+\rho}{2}+1}+A C(K) \sqrt{T} \lambda(w) \\
\leq A\left\|u_{0}\right\|_{s}+\sqrt{A C(K) T \lambda(w) \lambda(\Upsilon w)}+A C(K) \sqrt{T} \lambda(w)
\end{gathered}
$$

where, of course, we have used (30). Therefore,

$$
\begin{aligned}
& \lambda(\Upsilon w) \leq A\left\|u_{0}\right\|_{s}+\sqrt{A C(K) T \lambda(w) \lambda(\Upsilon w)}+A C(K) \sqrt{T} \lambda(w) \\
& \leq A\left\|u_{0}\right\|_{s}+\frac{1}{2} A C(K) T \lambda(w)+\frac{1}{2} \lambda(\Upsilon w)+A C(K) \sqrt{T} \lambda(w),
\end{aligned}
$$

which leads to

$$
\lambda(\Upsilon w) \leq 2 A\left\|u_{0}\right\|_{s}+A C(K) T \lambda(w)+2 A C(K) \sqrt{T} \lambda(w),
$$

that is, an estimate which is, by changing the constants and taking $T \leq 1$, of the form

$$
\lambda(\Upsilon w) \leq A\left\|u_{0}\right\|_{s}+A C(K) \sqrt{T} \lambda(w) .
$$

This is the main non linear estimate. In fact, when $u_{0} \neq 0$, by taking $K=2 A\left\|u_{0}\right\|_{s}$ for example, and then, $T>0$ such that

$$
T \leq\left(\frac{A\left\|u_{0}\right\|_{s}}{A C(K) K}\right)^{2}=\left(\frac{1}{2 A C(K)}\right)^{2},
$$

it follows from (33) that $\lambda(\Upsilon w) \leq K$ when $\lambda(w) \leq K$, that is, $\Upsilon(Z) \subset Z$. When $u_{0}=0$, it suffices to take $K>0$ and $T \leq 1 / A^{2} C(K)^{2}$ to obtain the same result.

Let us now show that $\Upsilon: Z \rightarrow Z$ is a contraction mapping. In fact, the arguments are similar to the above ones and we shall be brief. If $w_{1}, w_{2} \in Z$, then, $W=\Upsilon w_{1}-\Upsilon w_{2}$ satisfies the following Cauchy problem

$$
\left\{\begin{array}{l}
\partial_{t} W=i \mathscr{L} W+T_{b_{1}^{0}} \nabla_{x} W+T_{b_{2}^{0}} \nabla_{x} \bar{W}+T_{a_{1}^{0}} W+T_{a_{2}^{0}} \bar{W}+\widetilde{R}\left(z_{1}\right)-\widetilde{R}\left(z_{2}\right) \\
W(0)=0
\end{array}\right.
$$

where, as before, $z_{j}=\left(w_{j}, \nabla_{x} w_{j}, \bar{w}_{j}, \nabla_{x} \bar{w}_{j}\right), j=1,2$. Applying Theorem 3.1 to (34) gives

$$
\max \left\{\lambda_{1}(W)^{2}, \lambda_{2}(W)^{2}\right\} \leq A I_{T}\left(J^{s}\left(\widetilde{R}\left(z_{1}\right)-\widetilde{R}\left(z_{2}\right)\right), J^{s} W\right),
$$


and, consequently, we have to estimate uniformly in $\mu$ the integral

$$
\int_{0}^{T}\left|\left\langle G_{\mu} J^{s}\left(\widetilde{R}\left(z_{1}\right)-\widetilde{R}\left(z_{2}\right)\right), J^{s} W\right\rangle\right| d t
$$

It follows from (21) that

$$
\begin{aligned}
\widetilde{R}\left(z_{1}\right)-\widetilde{R}\left(z_{2}\right)= & T_{b_{1}\left(z_{1}\right)-b_{1}^{0}} \nabla\left(w_{1}-w_{2}\right)+T_{b_{1}\left(z_{1}\right)-b_{1}\left(z_{2}\right)} \nabla w_{2} \\
& +T_{b_{2}\left(z_{1}\right)-b_{2}^{0}} \nabla\left(\bar{w}_{1}-\bar{w}_{2}\right)+T_{b_{2}\left(z_{1}\right)-b_{2}\left(z_{2}\right)} \nabla \bar{w}_{2} \\
& +T_{a_{1}\left(z_{1}\right)-a_{1}^{0}}\left(w_{1}-w_{2}\right)+T_{a_{1}\left(z_{1}\right)-a_{1}\left(z_{2}\right)} w_{2} \\
& +T_{a_{2}\left(z_{1}\right)-a_{2}^{0}}\left(\bar{w}_{1}-\bar{w}_{2}\right)+T_{a_{2}\left(z_{1}\right)-a_{2}\left(z_{2}\right)} \bar{w}_{2} \\
& +R\left(z_{1}\right)-R\left(z_{2}\right)
\end{aligned}
$$

and we have to estimate the integral corresponding to each term of the above sum. Let us first consider the terms of the third line in (36). By the same argument as that used to obtain (27), we have

$\int_{0}^{T}\left|\left\langle G_{\mu} J^{s}\left(T_{a_{1}\left(z_{1}\right)-a_{1}^{0}}\left(w_{1}-w_{2}\right)+T_{a_{1}\left(z_{1}\right)-a_{1}\left(z_{2}\right)} w_{2}\right), J^{s} W\right\rangle\right| d t \leq A T C(K) \lambda\left(w_{1}-w_{2}\right) \lambda(W)$,

where we also applied Proposition 2.1 for the second term. Of course, we have the same estimate for the integral corresponding to the terms of the fourth line in (36).

As for the terms of the first line in (36), applying an argument similar to that yielding (28), one obtains

$$
\begin{aligned}
& \int_{0}^{T}\left|\left\langle G_{\mu} J^{s}\left(T_{b_{1}\left(z_{1}\right)-b_{1}^{0}} \nabla\left(w_{1}-w_{2}\right)+T_{b_{1}\left(z_{1}\right)-b_{1}\left(z_{2}\right)} \nabla w_{2}\right), J^{s} W\right\rangle\right| d t \\
& \leq A C(K) T\left(\lambda\left(w_{1}\right)^{2} \lambda_{2}\left(w_{1}-w_{2}\right)+\lambda\left(w_{1}-w_{2}\right)\left(\lambda\left(w_{1}\right)+\lambda\left(w_{2}\right)\right) \lambda_{2}\left(w_{2}\right)\right) \lambda_{2}(W) \\
& \leq A C(K) T\left(\lambda\left(w_{1}\right)^{2}+\lambda\left(w_{1}\right) \lambda\left(w_{2}\right)+\lambda\left(w_{2}\right)^{2}\right) \lambda\left(w_{1}-w_{2}\right) \lambda(W) \\
& \leq A C(K) K^{2} T \lambda\left(w_{1}-w_{2}\right) \lambda(W)
\end{aligned}
$$

and the same estimate holds for the terms of the second line in (36).

Last, for the terms of the fifth line in (36), applying Theorem 2.6 and estimating as in (29), we obtain

$$
\int_{0}^{T}\left|\left\langle G_{\mu} J^{s}\left(R\left(z_{1}\right)-R\left(z_{2}\right)\right), J^{s} W\right\rangle\right| d t \leq A \int_{0}^{T}\left\|z_{1}-z_{2}\right\|_{s-1}\|W\|_{s} d t
$$

$\leq A C(K) \int_{0}^{T}\left\|w_{1}-w_{2}\right\|_{s}\|W\|_{s} d t \leq A C(K) T \lambda_{1}\left(w_{1}-w_{2}\right) \lambda_{1}(W) \leq A C(K) T \lambda\left(w_{1}-w_{2}\right) \lambda(W)$. 
Summing up and going back to (35), we can conclude that

$$
\max \left\{\lambda_{1}(W)^{2}, \lambda_{2}(W)^{2}\right\} \leq A C(K) T \lambda\left(w_{1}-w_{2}\right) \lambda(W) .
$$

It remains to estimate $\lambda_{3}(W)$. Using the fact that $W$ satisfies the Cauchy problem (34) and an argument similar to that yielding (32), we obtain

$$
\begin{gathered}
\lambda_{3}(W) \leq A \lambda_{1}(W)+A C(K) \sqrt{T}\left(\lambda\left(w_{1}\right) \lambda_{1}\left(w_{1}-w_{2}\right)+\lambda\left(w_{1}-w_{2}\right) \lambda_{1}\left(w_{2}\right)\right)+A C(K) \sqrt{T} \lambda\left(w_{1}-w_{2}\right) \\
\leq A \lambda_{1}(W)+A C(K) \sqrt{T}\left(\lambda\left(w_{1}\right)+\lambda\left(w_{2}\right)\right) \lambda\left(w_{1}-w_{2}\right)+A C(K) \sqrt{T} \lambda\left(w_{1}-w_{2}\right) \\
\leq \sqrt{A C(K) T \lambda\left(w_{1}-w_{2}\right) \lambda(W)}+A C(K) \sqrt{T} \lambda\left(w_{1}-w_{2}\right) .
\end{gathered}
$$

Summing up, we have obtained

$$
\lambda(W) \leq \sqrt{A C(K) T \lambda\left(w_{1}-w_{2}\right) \lambda(W)}+A C(K) \sqrt{T} \lambda\left(w_{1}-w_{2}\right),
$$

hence,

$$
\lambda(W) \leq \frac{1}{2} A C(K) T \lambda\left(w_{1}-w_{2}\right)+\frac{1}{2} \lambda(W)+A C(K) \sqrt{T} \lambda\left(w_{1}-w_{2}\right),
$$

that is,

$$
\lambda(W)=\lambda\left(\Upsilon w_{1}-\Upsilon w_{2}\right) \leq A C(K) \sqrt{T} \lambda\left(w_{1}-w_{2}\right),
$$

with modified constants. This clearly implies, if $T$ is taken small enough, that $\Upsilon: Z \rightarrow Z$ is a contraction mapping and, thus, it has a unique fixed point $u$ in $Z$ which is a solution of (18). In fact, this is the solution of $(18)$ in $C\left([0, T], H^{s}\left(\mathbb{R}^{n}\right)\right)$ because the above method gives the local uniqueness and we obtain eventually the full uniqueness by applying a classical bootstrap argument. This proves the first part of Theorem 1.1.

The second part of Theorem 1.1 concerns the continuity of the solution operator $u_{0} \mapsto u$ and we start its proof by remarking that this operator maps bounded subsets of $H^{s}\left(\mathbb{R}^{n}\right)$ into bounded subsets of $C\left([0, T], H^{s}\left(\mathbb{R}^{n}\right)\right)$. In fact, if $B$ is a bounded subset of $H^{s}\left(\mathbb{R}^{n}\right)$, as remarked at the beginning of this section, the constant $A$ et the bounds of the semi-norms of the operators $G_{\mu}$ can be taken to depend only on $B$, that is, if $u_{0} \in B$, the estimates proven above and satisfied by $\Upsilon$ can be rewritten as

$$
\begin{aligned}
& \lambda(\Upsilon w) \leq A(B)\left\|u_{0}\right\|_{s}+A(B) C(K) \sqrt{T} \lambda(w), \\
& \lambda\left(\Upsilon w_{1}-\Upsilon w_{2}\right) \leq A(B) C(K) \sqrt{T} \lambda\left(w_{1}-w_{2}\right),
\end{aligned}
$$

where $A(B)$ depends only on $n, s$ and $B$, which implies that the constants $K$ and $T$ can be chosen depending only on $B$. Hence, for all $u_{0} \in B$, the associated solutions $u$ are all defined on the same interval $[0, T]$ and are all in the ball of radius $K$. As for the continuity, let $B$ be a bounded subset of $H^{s}\left(\mathbb{R}^{n}\right), u_{0}, u_{0}^{*} \in B, u, u^{*}$ the respective associated solutions and $w=u-u^{*}$. Then, $w$ satisfies the following Cauchy problem :

$$
\left\{\begin{array}{l}
\partial_{t} w=i \mathscr{L} w+D u-D^{*} u^{*}+\widetilde{R}-\widetilde{R}^{*}=i \mathscr{L} w+D w+\left(D-D^{*}\right) u^{*}+\widetilde{R}-\widetilde{R}^{*} \\
w(x, 0)=u_{0}(x)-u_{0}^{*}(x)
\end{array}\right.
$$


where $\quad D w=T_{b_{1}^{0}} \nabla w+T_{b_{2}^{0}} \nabla \bar{w}+T_{a_{1}^{0}} w+T_{a_{2}^{0}} \bar{w}, \quad D^{*} w=T_{b_{1}^{0, *}} \nabla w+T_{b_{2}^{0, *}} \nabla \bar{w}+T_{a_{1}^{0, *}} w+T_{a_{2}^{0, *}} \bar{w}$

$$
\widetilde{R}=\widetilde{R}(u, \nabla u, \bar{u}, \nabla \bar{u}) \quad \text { and } \quad \widetilde{R}^{*}=\widetilde{R}\left(u^{*}, \nabla u^{*}, \overline{u^{*}}, \nabla \overline{u^{*}}\right) .
$$

Of course, the $b_{j}^{0}, a_{j}^{0}$ correspond to $u_{0}$ whereas the $b_{j}^{0, *}, a_{j}^{0, *}$ correspond to $u_{0}^{*}$. Applying Theorem 3.1 gives us the inequality

$$
\max \left\{\lambda_{1}(w)^{2}, \lambda_{2}(w)^{2}\right\} \leq A(B)\left\|u_{0}-u_{0}^{*}\right\|_{s}^{2}+A(B) I_{T}\left(J^{s}\left(\left(D-D^{*}\right) u^{*}+\widetilde{R}-\widetilde{R}^{*}\right), J^{s} w\right),
$$

As it can be seen easily by going back to (21), we can write

$$
\begin{aligned}
\widetilde{R}-\widetilde{R}^{*}= & T_{b_{1}(u)-b_{1}^{0}} \nabla w+T_{b_{1}(u)-b_{1}\left(u^{*}\right)} \nabla u^{*}+T_{b_{1}^{0, *}-b_{1}^{0}} \nabla u^{*} \\
& +T_{b_{2}(u)-b_{2}^{0}} \nabla \bar{w}+T_{b_{2}(u)-b_{2}\left(u^{*}\right)} \nabla \overline{u^{*}}+T_{b_{2}^{0, *}-b_{2}^{0}} \nabla \overline{u^{*}} \\
& +T_{a_{1}(u)-a_{1}^{0}} w+T_{a_{1}(u)-a_{1}\left(u^{*}\right)} u^{*}+T_{a_{1}^{0, *}-a_{1}^{0}} u^{*} \\
& +T_{a_{2}(u)-a_{2}^{0}} \bar{w}+T_{a_{2}(u)-a_{2}\left(u^{*}\right)} \overline{u^{*}}+T_{a_{2}^{0, *}-a_{2}^{0}} \overline{u^{*}} \\
& +R(u, \nabla u, \bar{u}, \nabla \bar{u})-R\left(u^{*}, \nabla u^{*}, \overline{u^{*}}, \nabla \overline{u^{*}}\right)
\end{aligned}
$$

and we also have

$$
\left(D-D^{*}\right) u^{*}=T_{b_{1}^{0}-b_{1}^{0, *}} \nabla u^{*}+T_{b_{2}^{0}-b_{2}^{0, *}} \nabla \overline{u^{*}}+T_{a_{1}^{0}-a_{1}^{0, *}} u^{*}+T_{a_{2}^{0}-a_{2}^{0, *}} \overline{u^{*}} .
$$

Using the same arguments as before to estimate the integrals corresponding to each of the above terms yields

$$
\max \left\{\lambda_{1}(w)^{2}, \lambda_{2}(w)^{2}\right\} \leq A(B)\left\|u_{0}-u_{0}^{*}\right\|_{s}^{2}+A_{1}(B) C_{1}(K) T\left(\lambda(w)\left\|u_{0}-u_{0}^{*}\right\|_{s}+\lambda(w)^{2}\right),
$$

which becomes, after a change of the constants and assuming $T \leq 1$,

$$
\max \left\{\lambda_{1}(w), \lambda_{2}(w)\right\} \leq A(B)\left\|u_{0}-u_{0}^{*}\right\|_{s}+A(B) C(K) \sqrt{T} \lambda(w) .
$$

Next, using (39) and similar arguments, one can easily get

$$
\lambda_{3}(w) \leq A(B) C(K)\left(\left\|u_{0}-u_{0}^{*}\right\|_{s}+\lambda_{1}(w)\right),
$$

which becomes, after use of (43) and a possible change of the constants,

$$
\lambda_{3}(w) \leq A(B) C(K)\left(\left\|u_{0}-u_{0}^{*}\right\|_{s}+\sqrt{T} \lambda(w)\right) .
$$

Hence,

$$
\lambda(w) \leq A(B) C(K)\left\|u_{0}-u_{0}^{*}\right\|_{s}+A(B) C(K) \sqrt{T} \lambda(w),
$$

which, by taking $T \leq(1 / 2 A(B) C(K))^{2}$ (for example), leads to the Lipschitz estimate

$$
\lambda(w)=\lambda\left(u-u^{*}\right) \leq 2 A(B) C(K)\left\|u_{0}-u_{0}^{*}\right\|_{s},
$$

and this achieves the proof of Theorem 1.1. 


\section{$5 \quad$ Proof of Proposition 3.1}

We shall only give the main steps for the convenience of the reader and refer to [Bie] for the full details.

Let us start by remarking that it is sufficient to treat the case $s=0$. Indeed, if $v=J^{s} u$ and $v_{0}=J^{s} u_{0}$, it is easy to see that $u$ is a solution of (8) if and only if $v$ satisfies

$$
\left\{\begin{array}{l}
\partial_{t} v=i \mathscr{L} v+T_{b_{1}} \cdot \nabla_{x} v+T_{b_{2}} \cdot \nabla_{x} \bar{v}+\widetilde{C}_{1} v+\widetilde{C}_{2} \bar{v}+\widetilde{f}(x, t) \\
v(x, 0)=v_{0} \in L^{2}\left(\mathbb{R}^{n}\right)
\end{array}\right.
$$

where $\tilde{f}=J^{s} f$ and $\widetilde{C}_{k}=J^{s} C_{k} J^{-s}+\left[J^{s}, T_{b_{k}} . \nabla_{x}\right] J^{-s}, k=1$ ou 2 , and, thanks to the paradifferential calculus, the $\widetilde{C}_{k}$ are bounded operators in $L^{2}\left(\mathbb{R}^{n}\right)$.

The idea of proof is that of $[\mathrm{KePoVe}]$, inspired by [Tak], and consists in constructing a pseudodifferential operator $\mathbf{C}$ which is bounded and invertible in $L^{2}\left(\mathbb{R}^{n}\right)$ and to estimate $\sup _{[0, T]}\|\mathbf{C} u\|_{0}$ instead of estimating directly $\sup _{[0, T]}\|u\|_{0}$. Since $\frac{d}{d t}\langle\mathbf{C} u, \mathbf{C} u\rangle=\left\langle\mathbf{C} \partial_{t} u, \mathbf{C} u\right\rangle+$ $\left\langle\mathbf{C} u, \mathbf{C} \partial_{t} u\right\rangle$ and $u$ is a solution of (8), we obtain that

$$
\begin{aligned}
\frac{d}{d t}\|\mathbf{C} u\|_{0}^{2}=\langle i & \mathbf{C} \\
& \mathscr{L} u, \mathbf{C} u\rangle+\left\langle\mathbf{C} T_{b_{1}} \nabla u, \mathbf{C} u\right\rangle+\left\langle\mathbf{C} T_{b_{2}} \nabla \bar{u}, \mathbf{C} u\right\rangle \\
& +\left\langle\mathbf{C} C_{1} u, \mathbf{C} u\right\rangle+\left\langle\mathbf{C} C_{2} \bar{u}, \mathbf{C} u\right\rangle+\langle\mathbf{C} f, \mathbf{C} u\rangle \\
& +\langle\mathbf{C} u, i \mathbf{C} \mathscr{L} u\rangle+\left\langle\mathbf{C} u, \mathbf{C} T_{b_{1}} \nabla u\right\rangle+\left\langle\mathbf{C} u, \mathbf{C} T_{b_{2}} \nabla \bar{u}\right\rangle \\
& +\left\langle\mathbf{C} u, \mathbf{C} C_{1} u\right\rangle+\left\langle\mathbf{C} u, \mathbf{C} C_{2} \bar{u}\right\rangle+\langle\mathbf{C} u, \mathbf{C} f\rangle,
\end{aligned}
$$

and since

$$
\langle i \mathscr{L} \mathbf{C} u, \mathbf{C} u\rangle+\langle\mathbf{C} u, i \mathscr{L} \mathbf{C} u\rangle=0
$$

we have finally

$$
\begin{aligned}
\frac{d}{d t}\|\mathbf{C} u\|_{0}^{2}= & 2 \mathscr{R} e\left\langle\left(i[\mathbf{C}, \mathscr{L}]+\mathbf{C} T_{b_{1}} \nabla\right) u, \mathbf{C} u\right\rangle+2 \mathscr{R} e\left\langle\mathbf{C} T_{b_{2}} \nabla \bar{u}, \mathbf{C} u\right\rangle \\
& +2 \mathscr{R} e\langle\mathbf{C} u, \mathbf{C} f\rangle+2 \mathscr{R} e\left(\left\langle\mathbf{C} C_{1} u, \mathbf{C} u\right\rangle+\left\langle\mathbf{C} C_{2} \bar{u}, \mathbf{C} u\right\rangle\right) .
\end{aligned}
$$

The idea of $[\mathrm{KePoVe}]$ is precisely to choose $\mathbf{C}$ so that the operator $i[\mathbf{C}, \mathscr{L}]+\mathbf{C} T_{b_{1}} \nabla$ will be small in some sense. Here, we will make a refinement by writing $b_{1}=b_{1}^{\prime}+i b_{1}^{\prime \prime}$ with real $b_{1}^{\prime}$, $b_{1}^{\prime \prime}$, and by considering the operator $i[\mathbf{C}, \mathscr{L}]+i \mathbf{C} T_{b_{1}^{\prime \prime}} \nabla$ instead. This has been already used by $[\mathrm{Bie}]$ and essentially allows one to construct a real operator $\mathbf{C}$, that is, with the property $\overline{\mathbf{C} u}=\mathbf{C} \bar{u}$, which will be convenient in certain arguments. Now, clearly,

$$
\left|2 \mathscr{R} e\left(\left\langle\mathbf{C} C_{1} u, \mathbf{C} u\right\rangle+\left\langle\mathbf{C} C_{2} \bar{u}, \mathbf{C} u\right\rangle\right)\right| \leq 2\left(A_{1}+A_{2}\right)\|\mathbf{C}\|_{\mathscr{L}\left(L^{2}\right)}^{2}\|u\|_{0}^{2},
$$

and integrating on $\left[0, T^{\prime}\right], T^{\prime} \leq T$, yields

$$
\left\|\mathbf{C} u\left(T^{\prime}\right)\right\|_{2}^{2} \leq\left\|\mathbf{C} u_{0}\right\|_{0}^{2}+2\left|\mathscr{R} e \int_{0}^{T^{\prime}}\left\langle\left(i[\mathbf{C}, \mathscr{L}]+i \mathbf{C} T_{b_{1}^{\prime \prime}} \nabla\right) u, \mathbf{C} u\right\rangle d t\right|+2\left|\mathscr{R} e \int_{0}^{T^{\prime}}\left\langle\mathbf{C} T_{b_{1}^{\prime}} \nabla u, \mathbf{C} u\right\rangle d t\right|
$$




$$
+2\left|\mathscr{R} e \int_{0}^{T^{\prime}}\left\langle\mathbf{C} T_{b_{2}} \nabla \bar{u}, \mathbf{C} u\right\rangle d t\right|+2\left|\mathscr{R} e \int_{0}^{T^{\prime}}\langle\mathbf{C} u, \mathbf{C} f\rangle d t\right|+2\left(A_{1}+A_{2}\right)\|\mathbf{C}\|_{\mathscr{L}\left(L^{2}\right)}^{2} \int_{0}^{T^{\prime}}\|u(t)\|_{0}^{2} d t
$$

and our task will be to estimate appropriately each of the terms in the right hand side of this inequality. The most difficult one is

$$
\left|\int_{0}^{T}\left\langle\left(i[\mathbf{C}, \mathscr{L}]+i \mathbf{C} T_{b_{1}^{\prime \prime}} \nabla\right) u, \mathbf{C} u\right\rangle d t\right|
$$

and $\mathbf{C}$ will be constructed so that this term will be small with respect to some parameters to be defined later. To this end, let us denote by $\mathbf{c}$ the symbol of $\mathbf{C}$ and define

$$
p(x, \xi)=-2 \xi^{\sharp} \cdot \nabla_{x} \mathbf{c}(x, \xi)-\mathbf{c}(x, \xi) \tilde{b}_{1}^{\prime \prime}(x, \xi) \cdot \xi,
$$

where $\xi^{\sharp}=\left(\xi_{1}, \ldots, \xi_{j_{0}},-\xi_{j_{0}+1}, \ldots,-\xi_{n}\right)$ and $\tilde{b}_{1}^{\prime \prime}$ is such that $T_{b_{1}^{\prime \prime}}=\tilde{b}_{1}^{\prime \prime}(x, D)$; see $(2)$. The problem lies essentially in the fact that $p(x, \xi)$ is not the true principal symbol of the pseudodifferential (or paradifferential) operator $i[\mathbf{C}, \mathscr{L}]+i \mathbf{C} T_{b_{1}^{\prime \prime}} \nabla$ since $\mathbf{C}$ will be merely in the class $\mathrm{Op} S_{0,0}^{0}$. Nevertheless, the constructed $\mathbf{C}$ will allow us to obtain good estimates.

Set $\mathbf{c}(x, \xi)=\exp (\gamma(x, \xi))$ and $\gamma(x, \xi)=\sum_{\mu \in \mathbb{Z}^{n}} \alpha_{1, \mu} \gamma_{\mu}(x, \xi)$, where the $\alpha_{1, \mu}$ are the coefficients of $b_{1}$ in its decomposition with respect to the $\varphi_{1, \mu}$ (see Theorem 8), and the $\gamma_{\mu}(x, \xi)$ are defined a little later. Note here that one can assume the $\alpha_{1, \mu}$ real (and even non negative) without loss of generality. We can then write

$$
p(x, \xi)=\mathbf{c}(x, \xi) \sum_{\mu} \alpha_{1, \mu}\left(-2 \xi^{\sharp} \cdot \nabla_{x} \gamma_{\mu}(x, \xi)-\tilde{\varphi}_{1, \mu}(x, \xi) \cdot \xi\right),
$$

and this suggests to consider the following function

$$
\eta_{\mu}(x, \xi)=\frac{1}{2} \int_{0}^{\infty} \mathscr{I} m\left(\tilde{\varphi}_{1, \mu}\right)\left(x+s \xi^{\sharp}, \xi\right) \cdot \xi d s
$$

One can show that such a function is smooth and satisfies, for all multi-indices $\alpha, \beta$,

$$
\left|\partial_{x}^{\alpha} \partial_{\xi}^{\beta} \eta_{\mu}(x, \xi)\right| \leq A_{\alpha, \beta} \sup _{\beta^{\prime} \leq \beta}\left\|\langle\xi\rangle^{\left|\beta^{\prime}\right|} \partial_{x}^{\alpha} \partial_{\xi}^{\beta^{\prime}} \varphi_{1, \mu}\right\|_{L^{\infty}}\langle x-\mu\rangle^{|\beta|}\langle\xi\rangle^{-|\beta|},
$$

and, moreover,

$$
-2 \xi^{\sharp} \cdot \nabla_{x} \eta_{\mu}(x, \xi)-\mathscr{I}_{m}\left(\tilde{\varphi}_{1, \mu}\right)(x, \xi) \cdot \xi=0 .
$$

See $[\mathrm{KePoVe}]$ or $[\mathrm{Bie}]$ for the proof. To get an even function, we replace $\eta_{\mu}$ by

$$
\zeta_{\mu}(x, \xi)=\left(\eta_{\mu}(x, \xi)+\eta_{\mu}(x,-\xi)\right) / 2,
$$

which satisfies the same properties as $\eta_{\mu}$, and then set

$$
\gamma_{\mu}(x, \xi)=\theta\left(\frac{|\xi|}{R}\right) \psi\left(\frac{R\langle x-\mu\rangle}{\langle\xi\rangle}\right) \zeta_{\mu}(x, \xi),
$$

where $\theta$ and $\psi$ are smooth (real) functions on $\mathbb{R}$ such that $\theta(t)=1$ if $t \geq 2, \theta(t)=0$ if $t \leq 1$, $\psi(x)=1$ if $|t| \leq 1, \psi=0$ outside some compact set and $R$ is a large parameter that will 
be fixed later. One can easily check that $\gamma_{\mu} \in S_{0,0}^{0}$ and that its semi-norms are uniformly bounded with respect to $\mu$ and $R$. The following lemma gives the main properties of the operator $\mathbf{C}$ and its symbol

$$
\mathbf{c}(x, \xi)=\exp (\gamma(x, \xi))=\exp \left(\sum_{\mu} \alpha_{1, \mu} \gamma_{\mu}(x, \xi)\right) .
$$

Lemma $5.1(i)$ The symbol $\mathbf{c}(x, \xi)$ is real and even in $\xi$.

(ii) The symbol $\mathbf{c}(x, \xi)$ is in the class $S_{0,0}^{0}$. More precisely, for all $\alpha, \beta \in \mathbb{N}^{n}$,

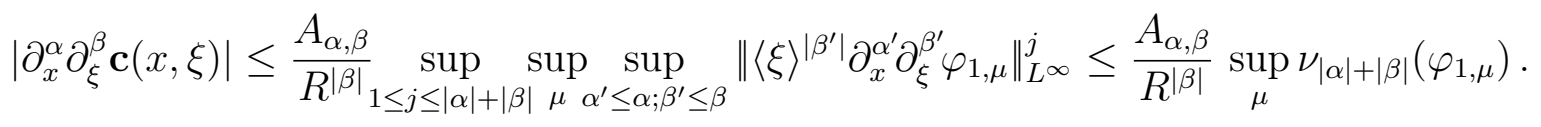

(iii) There exist $N \in \mathbb{N}$ and $A>0$ such that, for all $R \geq 1$ and all $v \in L^{2}\left(\mathbb{R}^{n}\right)$,

$$
\|\mathbf{C} v\|_{0} \leq A \sup \nu_{N}\left(\varphi_{1, \mu}\right)\|v\|_{0}
$$

$$
\text { and } \quad\|v\|_{0} \leq A \sup _{\mu} \nu_{N}\left(\varphi_{1, \mu}\right)\|\mathbf{C} v\|_{s}+\frac{A}{R} \sup _{\mu} \nu_{N}\left(\varphi_{1, \mu}\right)^{2}\|v\|_{s}
$$

(iv) The symbol $p(x, \xi)=-2 \xi^{\sharp} \cdot \nabla_{x} \mathbf{c}(x, \xi)-\mathbf{c}(x, \xi) \tilde{b}_{1}^{\prime \prime}(x, \xi) . \xi$ is in $S_{0,0}^{0}$ and its semi-norms (of order $\leq M$ ) are estimated by $A R \sup _{\mu} \nu_{M+1}\left(\varphi_{1, \mu}\right)$.

Even if here the function $\varphi_{1, \mu}$ is more general, the proof follows the same lines as that of [Bie] (Lemme 3.5 and Lemme 3.6) and we refer to it. These properties are sufficient to allow us to get the following estimates:

Lemma 5.2 Let $b(x, \xi)$ be a symbol satisfying

$$
\left\{\begin{array}{l}
b(x, \xi)=\sum_{\mu \in \mathbb{Z}^{n}} \alpha_{\mu} \varphi_{\mu}(x, \xi), \quad \varphi_{\mu} \in S_{1,0}^{0}, \quad \sum_{\mu}\left|\alpha_{\mu}\right| \leq A_{0}, \\
x \mapsto \varphi_{\mu}(x, \xi) \text { is rapidly decreasing in } x-\mu,
\end{array}\right.
$$

and let $\tilde{u}$ stand for $u$ or $\bar{u}$. Then, there exist $N \in \mathbb{N}$ and $A>0$ such that, for all $T>0$, $T^{\prime} \in[0, T], R \geq 1$ and every $H=h(x, D)$ in $\mathrm{Op} S_{0,0}^{0}$, the following estimates hold true :
(i) $\quad \int_{0}^{T^{\prime}}\left|\left\langle\left(\mathbf{C} T_{b} \nabla-(\mathbf{c} \tilde{b})(x, D) \nabla\right) \tilde{u}, H u\right\rangle\right| d t \leq \frac{A}{R}\|h\|_{C^{N}} \sup _{\mu} \nu_{N}\left(\varphi_{1, \mu}\right) \sup _{\mu}\left\|\varphi_{\mu}\right\|_{C^{N}}\left\|J^{\frac{1}{2}} u\right\|_{T}^{2}$.
(ii) $\int_{0}^{T^{\prime}}\left|\left\langle\left(i[\mathbf{C}, \mathscr{L}]+i \mathbf{C} T_{b_{1}^{\prime \prime}} \nabla\right) u, H u\right\rangle\right| d t \leq A\|h\|_{C^{N}} \sup _{\mu} \nu_{N}\left(\varphi_{1, \mu}\right)\left(R T \sup _{[0, T]}\|u\|_{0}^{2}+\frac{1}{R}\left\|J^{\frac{1}{2}} u\right\|_{T}^{2}\right)$.
(iii) $\int_{0}^{T^{\prime}}\left|\left\langle\left[\mathbf{C}, J^{s} T_{b} J^{-s} \nabla\right] \tilde{u}, H u\right\rangle\right| d t \leq A\|h\|_{C^{N}} \sup _{\mu} \nu_{N}\left(\varphi_{1, \mu}\right) \sup _{\mu}\left\|\varphi_{\mu}\right\|_{C^{N}}\left(T \sup _{[0, T]}\|u\|_{0}^{2}+\frac{\left\|J^{\frac{1}{2}} u\right\|_{T}^{2}}{R}\right)$.

Remark 1 The case $s \neq 0$ in (iii) is needed in Section $\%$ 
Proof : Using the pseudodifferential calculus, we can write the symbol $e(x, \xi)$ of the operator $E=\mathbf{C} T_{b} \nabla-(\mathbf{c} \tilde{b})(x, D) \nabla$ as $e=\sum_{\mu} \alpha_{\mu} e_{\mu}$ where $e_{\mu}$ is given by

$$
e_{\mu}(x, \xi)=\frac{1}{(2 \pi)^{n}} \sum_{j=1}^{n} \int_{0}^{1} \int \mathrm{e}^{-i y \eta} \partial_{\xi_{j}} \mathbf{c}(x, \xi+t \eta) \partial_{x_{j}} \tilde{\varphi}_{\mu}(x+y, \xi) \cdot \xi d y d \eta d t
$$

and we first remark that $e_{\mu} \in \mathrm{Op} S_{0,0}^{1}$ and that using the fast decrease of $\tilde{\varphi}_{\mu}(x, \xi)$ in $x-\mu$ and integrations by parts yields the fact that $e_{\mu}(x, \xi)$ is itself rapidly decreasing in $x-\mu$. Next, setting $E_{\mu}=e_{\mu}(x, D)$, we can write

$$
\begin{gathered}
\langle E \tilde{u}, H u\rangle=\sum_{\mu} \alpha_{\mu}\left\langle E_{\mu} \tilde{u}, H u\right\rangle=\sum_{\mu} \alpha_{\mu}\left\langle H^{*} E_{\mu} \tilde{u}, u\right\rangle \\
=\sum_{\mu} \alpha_{\mu}\left\langle\langle x-\mu\rangle^{\sigma_{0}} \tilde{H}\langle x-\mu\rangle^{-\sigma_{0}}\langle x-\mu\rangle^{\sigma_{0}} \tilde{E}_{\mu}\langle x-\mu\rangle^{\sigma_{0}} \tilde{u}_{\mu}, u_{\mu}\right\rangle
\end{gathered}
$$

where $\tilde{H}=J^{-1 / 2} H^{*} J^{1 / 2}, \quad \tilde{E}_{\mu}=J^{-1 / 2} E_{\mu} J^{-1 / 2}$ and $u_{\mu}=\langle x-\mu\rangle^{-\sigma_{0}} J^{1 / 2} u$. Now, it follows from the pseudodifferential calculus (Theorem 2.1) that $\tilde{H}$ and $\tilde{E}_{\mu}$ are in $\mathrm{Op} S_{0,0}^{0}$ and that we can estimate the semi-norms of $\tilde{H}$ and $\tilde{E}_{\mu}$ by those of $H$ and $E_{\mu}$ respectively. Moreover, it is easy to see that the symbol of $\tilde{E}_{\mu}$ inherits the fast decrease in $x-\mu$ which implies, by virtue of Lemma 2.1, that the operator $\langle x-\mu\rangle^{\sigma_{0}} \tilde{E}_{\mu}\langle x-\mu\rangle^{\sigma_{0}}$ is also in Op $S_{0,0}^{0}$ and that its semi-norms are estimated by those of $E_{\mu}$ uniformly in $\mu$. The same property hold for the operator $\langle x-\mu\rangle^{\sigma_{0}} \tilde{H}\langle x-\mu\rangle^{-\sigma_{0}}$ as it follows also from Lemma 2.1. This allows us to apply Calderon-Vaillancourt's theorem to obtain

$$
\begin{gathered}
\int_{0}^{T^{\prime}}|\langle E \tilde{u}, H u\rangle| d t \leq \sum_{\mu}\left|\alpha_{\mu}\right| \int_{0}^{T^{\prime}}\left\|\langle x-\mu\rangle^{\sigma_{0}} \tilde{H}\langle x-\mu\rangle^{-\sigma_{0}}\right\| \mathscr{L}\left(L^{2}\right)\left\|\langle x-\mu\rangle^{\sigma_{0}} \tilde{E}_{\mu}\langle x-\mu\rangle^{\sigma_{0}}\right\|_{\mathscr{L}\left(L^{2}\right)}\left\|u_{\mu}\right\|_{0}^{2} d t \\
\leq A\|h\|_{C^{N_{1}}} \sup _{\mu} \sum_{|\alpha|+|\beta| \leq N_{1}}\left\|\langle x-\mu\rangle^{2 \sigma_{0}} \partial_{x}^{\alpha} \partial_{\xi}^{\beta} e_{\mu}\right\|_{L^{\infty}}\left\|J^{\frac{1}{2}} u\right\|_{T}^{2} \\
\leq \frac{A}{R}\|h\|_{C^{N_{1}}} \sup _{\mu} \nu_{N_{2}}\left(\varphi_{1, \mu}\right) \sup _{\mu}\left\|\varphi_{\mu}\right\|_{C^{N_{2}}}\left\|J^{\frac{1}{2}} u\right\|_{T}^{2}
\end{gathered}
$$

which proves $(i)$.

To prove $(i i)$, note first that the symbol of $i[\mathbf{C}, \mathscr{L}]$ is given by

$$
-2 \xi^{\sharp} \cdot \nabla_{x} \mathbf{c}(x, \xi)+\left(\mathscr{L}_{x} \mathbf{c}\right)(x, \xi)
$$

and that of $i \mathbf{C} T_{b_{1}^{\prime \prime}} \nabla$ can be written as

$$
i \mathbf{c}(x, \xi) \tilde{b}_{1}^{\prime \prime}(x, \xi) . i \xi+\frac{1}{(2 \pi)^{n}} \sum_{j=1}^{n} \int_{0}^{1} \int \mathrm{e}^{-i y \eta} \partial_{\xi_{j}} \mathbf{c}(x, \xi+t \eta) \partial_{x_{j}} \tilde{b}_{1}^{\prime \prime}(x+y, \xi) . i \xi d y d \eta d t .
$$

Thus, the symbol of the operator $i[\mathbf{C}, \mathscr{L}]+i \mathbf{C} T_{b_{1}^{\prime \prime}} \nabla$ is given by

$$
\left(\mathscr{L}_{x} \mathbf{c}\right)(x, \xi)+p(x, \xi)+i e(x, \xi)
$$


where $p(x, \xi)$ is given by (49), $e=\sum_{\mu} \alpha_{\mu} e_{\mu}$ and $e_{\mu}(x, \xi)$ is given by (53) with $\alpha_{\mu}=\alpha_{1, \mu}$ and $\varphi_{\mu}=\mathscr{I}_{m}\left(\varphi_{1, \mu}\right)$. Hence, applying Lemma 5.1 and Calderon-Vaillancourt's theorem yields the estimate

$$
\int_{0}^{T^{\prime}}\left|\left\langle\left(\left(\mathscr{L}_{x} \mathbf{c}\right)(x, D)+p(x, D)\right) u, H u\right\rangle\right| d t \leq A R T\|h\|_{C^{N_{1}}} \sup _{\mu} \nu_{N_{1}}\left(\varphi_{1, \mu}\right)^{2} \sup _{[0, T]}\|u\|_{0}^{2},
$$

and applying part $(i)$ gives the estimate

$$
\int_{0}^{T^{\prime}} \mid\langle(i e(x, D) u, H u\rangle| d t \leq \frac{A}{R}\|h\|_{C^{N_{2}}} \sup _{\mu} \nu_{N_{2}}\left(\varphi_{1, \mu}\right)^{2}\left\|J^{\frac{1}{2}} u\right\|_{T}^{2},
$$

which proves $(i i)$.

To prove $\left(\right.$ iii), we first treat the case $s=0$ and note that the symbol of $\left[\mathbf{C}, T_{b} \nabla\right]=$ $\mathbf{C} T_{b} \nabla-T_{b} \nabla \mathbf{C}$ can be written simply as $e(x, \xi)-e_{0}(x, \xi)$ where $e(x, \xi)$ is the symbol of the operator $E$ studied in $(i)$ and

$$
e_{0}(x, \xi)=\frac{1}{(2 \pi)^{n}} \sum_{j=1}^{n} \int_{0}^{1} \int \mathrm{e}^{-i y \eta} \partial_{\xi_{j}}(\tilde{b}(x, \xi+t \eta) \cdot(\xi+t \eta)) \partial_{x_{j}} \mathbf{c}(x+y, \xi) d y d \eta d t
$$

Since $\partial_{\xi_{j}}(\tilde{b}(x, \xi) . \xi)$ is of order 0 , the symbol $e_{0}(x, \xi)$ is in fact in $S_{0,0}^{0}$ and the semi-norms of $e_{0}$ are estimated by a product of semi-norms of $\tilde{b}$ and $\mathbf{c}$. Hence, by using the decomposition of $b$ as above, we get

$$
\int_{0}^{T^{\prime}}\left|\left\langle e_{0}(x, D) \tilde{u}, H u\right\rangle\right| d t \leq A T\|h\|_{C^{N_{1}}} \sup _{\mu}\left\|\varphi_{\mu}\right\|_{C^{N_{2}}} \sup _{\mu} \nu_{N_{2}}\left(\varphi_{1, \mu}\right) \sup _{[0, T]}\|u\|_{0}^{2},
$$

which, together with (54), yields (iii) in the case $s=0$. If $s \neq 0$, it follows from the pseudodifferential and paradifferential calculi that $J^{s} T_{b} J^{-s}=T_{b \#}$ where $b^{\#}=\sum_{\mu} \alpha_{\mu} \psi_{\mu}$ and $\psi_{\mu}$ is given by

$$
\psi_{\mu}(x, \xi)=\frac{1}{(2 \pi)^{n}} \int \mathrm{e}^{-i y \eta}\langle\xi+\eta\rangle^{s} \varphi_{\mu}(x+y, \xi)\langle\xi\rangle^{-s} d y d \eta
$$

which implies that $\psi_{\mu}$ is also rapidly decreasing in $x-\mu$ and that it is in $S_{1,0}^{0}$ with semi-norms estimated by those of $\varphi_{\mu}$. This shows that the the case $s \neq 0$ follows from the case $s=0$ and achieves the proof of Lemma 5.2.

Lemma 5.3 Let $b$ be as in the preceding lemma. Then, there exist $N \in \mathbb{N}$ and $A>0$ such that, for all $T>0, T^{\prime} \in[0, T]$ and $R \geq 1$, the following estimates hold true:

(i) If $b(x, \xi)$ is even in $\xi$, then,

$$
\int_{0}^{T^{\prime}}\left|\left\langle\mathbf{C} T_{b} \nabla \bar{u}, \mathbf{C} u\right\rangle\right| d t \leq A \sup _{\mu} \nu_{N}\left(\varphi_{1, \mu}\right) \sup _{\mu}\left\|\varphi_{\mu}\right\|_{C^{N}}\left(T \sup _{0 \leq t \leq T}\|u\|_{0}^{2}+\frac{1}{R}\left\|J^{\frac{1}{2}} u\right\|_{T}\right) .
$$

(ii) If $b$ is real, then,

$$
\left|\mathscr{R} e \int_{0}^{T^{\prime}}\left\langle\mathbf{C} T_{b} \nabla u, \mathbf{C} u\right\rangle d t\right| \leq A \sup _{\mu} \nu_{N}\left(\varphi_{1, \mu}\right) \sup _{\mu}\left\|\varphi_{\mu}\right\|_{C^{N}}\left(T \sup _{0 \leq t \leq T}\|u\|_{0}^{2}+\frac{1}{R}\left\|J^{\frac{1}{2}} u\right\|_{T}\right) .
$$


Proof : Since $\mathbf{C}$ is real, we can write

$$
\left\langle\mathbf{C} T_{b} \nabla \bar{u}, \mathbf{C} u\right\rangle=\left\langle T_{b} \nabla \mathbf{C} \bar{u}, \mathbf{C} u\right\rangle+\left\langle\left[\mathbf{C}, T_{b} \nabla\right] \bar{u}, \mathbf{C} u\right\rangle=\left\langle T_{b} \nabla \overline{\mathbf{C} u}, \mathbf{C} u\right\rangle+\left\langle\left[\mathbf{C}, T_{b} \nabla\right] \bar{u}, \mathbf{C u}\right\rangle .
$$

Now, the integral corresponding to $\left\langle\left[\mathbf{C}, T_{b} \nabla\right] \bar{u}, \mathbf{C} u\right\rangle$ is treated by Lemma 5.2(iii). As for the other term, we note that it is of the form $\left\langle T_{b} \nabla \bar{v}, v\right\rangle$, so, it suffices to study such a term. Since $b(x, \xi)$ is even in $\xi$, we have

$$
\left\langle T_{b} \nabla \bar{v}, v\right\rangle=\overline{\left\langle v, T_{b} \nabla \bar{v}\right\rangle}=\left\langle\bar{v}, T_{\bar{b}} \nabla v\right\rangle=\left\langle\left(T_{\bar{b}} \nabla\right)^{*} \bar{v}, v\right\rangle
$$

and it follows from the pseudodifferential (or paradifferential) calculus that

$$
\left(T_{\bar{b}} \nabla\right)^{*}=-T_{b} \nabla+E_{1}
$$

where $E_{1}$ is of type $S_{1,0}^{0}$ and its semi-norms (up to some finite order) are estimated by those of $b$. Hence,

$$
\left\langle T_{b} \nabla \bar{v}, v\right\rangle=-\left\langle T_{b} \nabla \bar{v}, v\right\rangle+\left\langle E_{1} \bar{v}, v\right\rangle
$$

and $\left\langle T_{b} \nabla \bar{v}, v\right\rangle=\frac{1}{2}\left\langle E_{1} \bar{v}, v\right\rangle$, that is, $\left\langle T_{b} \nabla \overline{\mathbf{C} u}, \mathbf{C} u\right\rangle=\frac{1}{2}\left\langle E_{1} \overline{\mathbf{C} u}, \mathbf{C} u\right\rangle$, and $(i)$ follows just by applying Calderon-Vaillancourt's theorem and Lemma 5.1.

To prove $(i i)$, we write as before

$$
\left\langle\mathbf{C} T_{b} \nabla u, \mathbf{C} u\right\rangle=\left\langle T_{b} \nabla \mathbf{C} u, \mathbf{C} u\right\rangle+\left\langle\left[\mathbf{C}, T_{b} \nabla\right] u, \mathbf{C} u\right\rangle,
$$

and then apply Lemma $5.2(i i i)$ to reduce the problem to the study of $\mathscr{R} e\left\langle T_{b} \nabla \mathbf{C} u, \mathbf{C} u\right\rangle$. Now, it follows from (55) and the fact that $b$ is real that we have

$2 \mathscr{R} e\left\langle T_{b} \nabla \mathbf{C} u, \mathbf{C} u\right\rangle=\left\langle T_{b} \nabla \mathbf{C} u, \mathbf{C} u\right\rangle+\left\langle\mathbf{C} u, T_{b} \nabla \mathbf{C} u\right\rangle=\left\langle\left(T_{b} \nabla+\left(T_{b} \nabla\right)^{*}\right) \mathbf{C} u, \mathbf{C} u\right\rangle=\left\langle E_{1} \mathbf{C} u, \mathbf{C} u\right\rangle$

and the proof ends like that of $(i)$. The lemma is thus proved.

It is clear now that applying Lemma 5.1, Lemma 5.2 and Lemma 5.3 to the inequality (48) yields Proposition 3.1.

\section{$6 \quad$ Proof of Proposition 3.2}

By the same argument as that used in the beginning of the proof of Proposition 3.1, it is sufficient to establish the first estimate in the case $s=0$.

The proof follows the same ideas as that of $[\mathrm{KePoVe}]$ or [Bie]. The difference is that here the $T_{b_{k}}, k=1,2$, are general paradifferential operators of order 0 instead of merely multiplication or paramultiplication operators.

Since

$$
\partial_{t} u=i \mathscr{L} u+T_{b_{1}} \cdot \nabla u+T_{b_{2}} \cdot \nabla \bar{u}+C_{1} u+C_{2} \bar{u}+f
$$

and

$$
\partial_{t} \bar{u}=-i \mathscr{L} \bar{u}+T_{\bar{b}_{1}} \cdot \nabla \bar{u}+T_{\bar{b}_{2}} \cdot \nabla u+\bar{C}_{1} \bar{u}+\bar{C}_{2} u+\bar{f}
$$


where the operators $\bar{C}_{k}$ are defined by $\bar{C}_{k} u=\overline{C_{k} \bar{u}}$, one starts by remarking that the vector unknown $w=\left(\begin{array}{l}u \\ \bar{u}\end{array}\right)$ satisfies the following system

$$
\partial_{t} w=i H w+B w+C w+F
$$

where

$$
H=\left(\begin{array}{cc}
\mathscr{L} & 0 \\
0 & -\mathscr{L}
\end{array}\right), \quad B=\left(\begin{array}{cc}
T_{b_{1}} \nabla & T_{b_{2}} \nabla \\
T_{\bar{b}_{2}} \nabla & T_{\bar{b}_{1}} \nabla
\end{array}\right), \quad C=\left(\begin{array}{cc}
C_{1} & C_{2} \\
\bar{C}_{1} & \bar{C}_{2}
\end{array}\right), \quad F=\left(\begin{array}{c}
f \\
\bar{f}
\end{array}\right),
$$

and the idea then is to estimate the expression $\langle\Psi w, w\rangle$ by means of Gårding's inequality for systems via Doi's argument. Here,

$$
\Psi=\left(\begin{array}{cc}
\Psi_{0} & 0 \\
0 & -\Psi_{0}
\end{array}\right)
$$

and $\Psi_{0}$ is an appropriate pseudodifferential operator in $\mathrm{Op} S_{1,0}^{0}$ to be chosen a little later. By using (56), one gets easily

$$
\begin{aligned}
\partial_{t}\langle\Psi w, w\rangle & =\left\langle\Psi \partial_{t} w, w\right\rangle+\left\langle\Psi w, \partial_{t} w\right\rangle \\
& =\left\langle\left(i[\Psi, H]+B^{*} \Psi+\Psi B+C^{*} \Psi+\Psi C\right) w, w\right\rangle+\langle\Psi F, w\rangle+\langle\Psi w, F\rangle
\end{aligned}
$$

and, as one can check also easily, the principal symbol of the first order operator

$$
i[\Psi, H]+B^{*} \Psi+\Psi B+C^{*} \Psi+\Psi C
$$

is given by

$$
M(x, \xi)=\left(\begin{array}{rr}
2 \xi^{\sharp} \cdot \nabla_{x} \psi_{0}(x, \xi)-2 \xi . \mathscr{I}_{m}\left(\tilde{b}_{1}\right)(x, \xi) \psi_{0}(x, \xi) & 2 i \xi . \tilde{b}_{2}(x, \xi) \psi_{0}(x, \xi) \\
-2 i \xi . \overline{\tilde{b}}_{2}(x, \xi) \psi_{0}(x, \xi) & 2 \xi^{\sharp} \cdot \nabla_{x} \psi_{0}(x, \xi)-2 \xi . \mathscr{I}_{m}\left(\tilde{b}_{1}\right)(x, \xi) \psi_{0}(x, \xi)
\end{array}\right)
$$

where $\psi_{0}$ denotes the symbol of $\Psi_{0}$. Now, for $\psi_{0}$, we shall make the following choice which follows the idea of Doi (see [Doi]). Define

$$
\begin{gathered}
p(x, \xi)=\langle\xi\rangle^{-1} \sum_{j=1}^{n} \xi_{j}^{\sharp} h\left(x_{j}\right) \quad \text { with } \quad h(t)=\int_{0}^{t}\langle s\rangle^{-2 \sigma_{0}} d s, \\
p_{\mu}(x, \xi)=p(x-\mu, \xi)+A_{0} \sum_{\mu^{\prime} \in \mathbb{Z}^{n}}\left(\left|\alpha_{1, \mu^{\prime}}\right|+\left|\alpha_{2, \mu^{\prime}}\right|\right) p\left(x-\mu^{\prime}, \xi\right), \\
\text { and } \psi_{0}(x, \xi)=\psi_{\mu}(x, \xi)=\exp \left(-p_{\mu}(x, \xi)\right) .
\end{gathered}
$$

Here, the $\alpha_{1, \mu^{\prime}}$ and $\alpha_{2, \mu^{\prime}}$ are the coefficients of $b_{1}$ and $b_{2}$ in their decompositions with respect to the $\varphi_{1, \mu^{\prime}}$ and $\varphi_{2, \mu^{\prime}}$ respectively (see Theorem 8), $A_{0}$ is a large constant that will be 
determined later and $\mu \in \mathbb{Z}^{n}$ is fixed for the moment. However, from now on, we shall write $\Psi_{\mu}$ and $\psi_{\mu}$ instead of $\Psi_{0}$ and $\psi_{0}$ to emphasize the dependance on $\mu$. First, note that $p_{\mu}$ and $\psi_{\mu}$ are in $S_{1,0}^{0}$ and that their semi-norms are uniformly bounded with respect to $\mu$. Next, with these notations, the symbol $M(x, \xi)$ can be rewritten as

$$
M(x, \xi)=2 \psi_{\mu}(x, \xi)\left(\begin{array}{rr}
-\xi^{\sharp} \cdot \nabla_{x} p_{\mu}(x, \xi)-\xi . \mathscr{I}_{m}\left(\tilde{b}_{1}\right)(x, \xi) & i \xi . \tilde{b}_{2}(x, \xi) \\
-i \xi . \tilde{\tilde{b}}_{2}(x, \xi) & -\xi^{\sharp} \cdot \nabla_{x} p_{\mu}(x, \xi)-\xi \cdot \mathscr{I}_{m}\left(\tilde{b}_{1}\right)(x, \xi)
\end{array}\right) .
$$

Consider now the matrix $Z(x, \xi)=-M(x, \xi)-V(x, \xi)$ where

$$
V(x, \xi)=\frac{2 \psi_{\mu}(x, \xi)|\xi|^{2}}{\langle\xi\rangle\langle x-\mu\rangle^{2 \sigma_{0}}}\left(\begin{array}{ll}
1 & 0 \\
0 & 1
\end{array}\right) .
$$

$Z(x, \xi)$ is a matrix of symbols in $S_{1,0}^{1}$ and, in order to apply Gårding's inequality, we are going to show that, for large $\xi$, it is a non negative matrix, that is, $\langle Z(x, \xi) v, v\rangle \geq 0, \forall v \in \mathbb{C}^{2}$. In fact, $Z(x, \xi)$ is of the form

$$
2 \psi_{\mu}(x, \xi)\left(\begin{array}{cc}
\alpha & \beta \\
\bar{\beta} & \alpha
\end{array}\right)
$$

where

$$
\alpha=\xi^{\sharp} \cdot \nabla_{x} p_{\mu}(x, \xi)-\frac{|\xi|^{2}}{\langle\xi\rangle\langle x-\mu\rangle^{2 \sigma_{0}}}+\xi . \mathscr{I}_{m}\left(\tilde{b}_{1}\right)(x, \xi) \quad \text { and } \quad \beta=-i \xi . \tilde{b}_{2}(x, \xi),
$$

and it is sufficient to show that the two eigenvalues $\alpha \pm|\beta|$ of $\left(\begin{array}{ll}\alpha & \beta \\ \bar{\beta} & \alpha\end{array}\right)$ are non negative, or, equivalently, that $\alpha \geq|\beta|$, that is,

$$
\xi^{\sharp} \cdot \nabla_{x} p_{\mu}(x, \xi)-\frac{|\xi|^{2}}{\langle\xi\rangle\langle x-\mu\rangle^{2 \sigma_{0}}}+\xi . \mathscr{I}_{m}\left(\tilde{b}_{1}\right)(x, \xi) \geq\left|-i \xi . \tilde{b}_{2}(x, \xi)\right| .
$$

Now, the main reason for the choice of the symbol $p_{\mu}$ is that it allows to get the following inequality :

$$
\begin{aligned}
\xi^{\sharp} \cdot \nabla_{x} p_{\mu}(x, \xi) & =\xi^{\sharp} \cdot \nabla_{x} p(x-\mu, \xi)+A_{0} \sum_{\mu^{\prime} \in \mathbb{Z}^{n}}\left(\left|\alpha_{1, \mu^{\prime}}\right|+\left|\alpha_{2, \mu^{\prime}}\right|\right) \xi^{\sharp} \cdot \nabla_{x} p\left(x-\mu^{\prime}, \xi\right) \\
& =\sum_{j=1}^{n} \frac{\xi_{j}^{2}}{\langle\xi\rangle\left\langle x_{j}-\mu_{j}\right\rangle^{2 \sigma_{0}}}+A_{0} \sum_{\mu^{\prime} \in \mathbb{Z}^{n}}\left(\left|\alpha_{1, \mu^{\prime}}\right|+\left|\alpha_{2, \mu^{\prime}}\right|\right) \sum_{j=1}^{n} \frac{\xi_{j}^{2}}{\langle\xi\rangle\left\langle x_{j}-\mu_{j}^{\prime}\right\rangle^{2 \sigma_{0}}} \\
& \geq \frac{|\xi|^{2}}{\langle\xi\rangle\langle x-\mu\rangle^{2 \sigma_{0}}}+A_{0} \sum_{\mu^{\prime} \in \mathbb{Z}^{n}}\left(\left|\alpha_{1, \mu^{\prime}}\right|+\left|\alpha_{2, \mu^{\prime}}\right|\right) \frac{|\xi|^{2}}{\langle\xi\rangle\left\langle x-\mu^{\prime}\right\rangle^{2 \sigma_{0}}},
\end{aligned}
$$

that is,

$$
\xi^{\sharp} \cdot \nabla_{x} p_{\mu}(x, \xi)-\frac{|\xi|^{2}}{\langle\xi\rangle\langle x-\mu\rangle^{2 \sigma_{0}}} \geq A_{0} \sum_{\mu^{\prime} \in \mathbb{Z}^{n}}\left(\left|\alpha_{1, \mu^{\prime}}\right|+\left|\alpha_{2, \mu^{\prime}}\right|\right) \frac{|\xi|^{2}}{\langle\xi\rangle\left\langle x-\mu^{\prime}\right\rangle^{2 \sigma_{0}}} .
$$

Besides, we have

$$
\tilde{b}_{k}(x, \xi)=\sum_{\mu^{\prime} \in \mathbb{Z}^{n}} \alpha_{k, \mu^{\prime}} \tilde{\varphi}_{k, \mu^{\prime}}(x, \xi), \quad k=1,2,
$$


and it follows from Lemma 2.3 that

$$
\left\langle x-\mu^{\prime}\right\rangle^{2 \sigma_{0}}\left|\tilde{\varphi}_{k, \mu^{\prime}}(x, \xi)\right| \leq A(n),
$$

with a constant $A(n)$ which depends only on the dimension. Hence,

$$
\left|i \xi . \tilde{b}_{k}(x, \xi)\right| \leq A(n) \sum_{\mu^{\prime} \in \mathbb{Z}^{n}}\left|\alpha_{k, \mu^{\prime}}\right| \frac{|\xi|}{\left\langle x-\mu^{\prime}\right\rangle^{2 \sigma_{0}}} \leq \sqrt{2} A(n) \sum_{\mu^{\prime} \in \mathbb{Z}^{n}}\left|\alpha_{k, \mu^{\prime}}\right| \frac{|\xi|^{2}}{\langle\xi\rangle\left\langle x-\mu^{\prime}\right\rangle^{2 \sigma_{0}}}, \quad k=1,2,
$$

if $|\xi| \geq 1$, which, together with (60), implies (58) by taking $A_{0} \geq \sqrt{2} A(n)$. Thus, the matrix symbol $Z(x, \xi)$ is non negative, and since it is also hermitian, $Z(x, \xi)+Z(x, \xi)^{*}$ is also non negative and we can apply Gårding's inequality for systems :

$$
\mathscr{R} e\langle Z(x, D) w, w\rangle \geq-A\left(1+\sup _{|\alpha|+|\beta| \leq N} \sup _{k, \mu^{\prime}}\left\|\langle\xi\rangle^{|\beta|} \partial_{x}^{\alpha} \partial_{\xi}^{\beta} \varphi_{k, \mu^{\prime}}\right\|_{L^{\infty}}\right)\|w\|_{0}^{2}
$$

where the constant $A$ depends only on $A_{1}, A_{2}$ and the dimension $n$ and the integer $N$ depends only on the dimension $n$. Now, going back to (57), we can rewrite it as

$$
\partial_{t}\langle\Psi w, w\rangle=\langle(-Z(x, D)-V(x, D)+E) w, w\rangle+\langle\Psi F, w\rangle+\langle\Psi w, F\rangle
$$

where $E$ is a bounded operator in $L^{2}\left(\mathbb{R}^{n}\right)$, and integrating it on $[0, T]$ yields

$$
\begin{gathered}
\int_{0}^{T}\langle V(x, D) w, w\rangle d t=\langle\Psi w(0), w(0)\rangle-\langle\Psi w(T), w(T)\rangle-\int_{0}^{T}\langle Z(x, D) w, w\rangle d t \\
+\int_{0}^{T}\langle E w, w\rangle d t+\int_{0}^{T}\langle\Psi F, w\rangle d t+\int_{0}^{T}\langle\Psi w, F\rangle d t .
\end{gathered}
$$

Taking the real part, using (61) and estimating, we obtain

$$
\begin{gathered}
\mathscr{R} e \int_{0}^{T}\langle V(x, D) w, w\rangle d t \leq A \sup _{[0, T]}\|w\|_{0}^{2}+A T\left(1+\sup _{k, \mu^{\prime}} \nu_{N}\left(\varphi_{k, \mu^{\prime}}\right)\right) \sup _{[0, T]}\|w\|_{0}^{2} \\
+\left|\int_{0}^{T}\langle\Psi F, w\rangle d t\right|+\left|\int_{0}^{T}\langle\Psi w, F\rangle d t\right|
\end{gathered}
$$

and since $\psi_{\mu}(x, \xi) \geq \exp (-A)$ and, for $|\xi| \geq 1$,

$$
V(x, \xi) \geq \mathrm{e}^{-A} \frac{\langle\xi\rangle}{\langle x-\mu\rangle^{2 \sigma_{0}}}\left(\begin{array}{ll}
1 & 0 \\
0 & 1
\end{array}\right)
$$

a second application of Gårding inequality gives us

$$
\begin{gathered}
\mathscr{R e} \int_{0}^{T}\left\langle J^{1 / 2}\langle x-\mu\rangle^{-2 \sigma_{0}} J^{1 / 2} w, w\right\rangle d t \leq A \sup _{[0, T]}\|w\|_{0}^{2}\left(1+T+T \sup _{k, \mu^{\prime}} \nu_{N}\left(\varphi_{k, \mu^{\prime}}\right)\right) \\
+\left|\int_{0}^{T}\langle\Psi F, w\rangle d t\right|+\left|\int_{0}^{T}\langle\Psi w, F\rangle d t\right|,
\end{gathered}
$$


with a modified constant $A$. Since we can write $\langle\Psi F, w\rangle=\left\langle\Psi_{\mu} f, u\right\rangle-\overline{\left\langle\bar{\Psi}_{\mu} f, u\right\rangle}$ and a similar expression for $\langle\Psi w, F\rangle$, by going back to $u$, we get eventually

$$
\begin{gathered}
\int_{0}^{T}\left\|\langle x-\mu\rangle^{-\sigma_{0}} J^{1 / 2} u\right\|_{0}^{2} d t \leq A \sup _{[0, T]}\|u\|_{0}^{2}\left(1+T+T \sup _{k, \mu^{\prime}} \nu_{N}\left(\varphi_{k, \mu^{\prime}}\right)\right) \\
+\int_{0}^{T}\left|\left\langle\Psi_{\mu} f, u\right\rangle\right| d t+\int_{0}^{T}\left|\left\langle\bar{\Psi}_{\mu} f, u\right\rangle\right| d t+\int_{0}^{T}\left|\left\langle\Psi_{\mu}^{*} f, u\right\rangle\right| d t+\int_{0}^{T}\left|\left\langle\bar{\Psi}_{\mu}^{*} f, u\right\rangle\right| d t,
\end{gathered}
$$

which yields the first part of Proposition 3.2 by taking the supremum over all $\mu \in \mathbb{Z}^{n}$.

As for the second estimate of Proposition 3.2, we first remark that, since $\mathbf{C}$ is real, $\mathbf{C} u$ satisfies

$$
\partial_{t} \mathbf{C} u=i \mathscr{L} \mathbf{C} u+T_{b_{1}^{\prime}} \cdot \nabla \mathbf{C} u+T_{b_{2}} \cdot \nabla \overline{\mathbf{C} u}+C_{1} \mathbf{C} u+C_{2} \overline{\mathbf{C} u}+\tilde{f}
$$

where $k=1,2, b_{1}=b_{1}^{\prime}+i b_{1}^{\prime \prime}$ with real $b_{1}^{\prime}, b_{1}^{\prime \prime}$, and

$$
\tilde{f}=\left(i[\mathbf{C}, \mathscr{L}]+\mathbf{C} T_{i b_{1}^{\prime \prime}} \nabla\right) u+\left[\mathbf{C}, T_{b_{1}^{\prime}} \cdot \nabla\right] u+\left[\mathbf{C}, T_{b_{2}} \cdot \nabla\right] \bar{u}+\left[\mathbf{C}, C_{1}\right] u+\left[\mathbf{C}, C_{2}\right] \bar{u}+\mathbf{C} f .
$$

Hence, we can apply the first estimate of Proposition 3.2 to $\mathbf{C} u$ obtaining

$$
\left\|J^{s+1 / 2} \mathbf{C} u\right\|_{T}^{2} \leq A\left(1+T+T \sup _{k, \mu} \nu_{N}\left(\varphi_{k, \mu}\right)\right) \sup _{[0, T]}\|\mathbf{C} u\|_{s}^{2}+\sum_{j=1}^{4} \sup _{\mu} \int_{0}^{T}\left|\left\langle\Psi_{j, \mu} J^{s} \widetilde{f}, J^{s} \mathbf{C} u\right\rangle\right| d t
$$

where $\Psi_{j, \mu}=\psi_{j}(x-\mu, D)$. Thus, we are led to estimate essentially the following terms

$$
\begin{gathered}
\int_{0}^{T}\left|\left\langle J^{s}\left(i[\mathbf{C}, \mathscr{L}]+\mathbf{C} T_{i b_{1}^{\prime \prime}} \nabla\right) u, \Psi_{j, \mu}^{*} J^{s} \mathbf{C} u\right\rangle\right| d t+\int_{0}^{T}\left|\left\langle J^{s}\left[\mathbf{C}, T_{b_{1}^{\prime}} \cdot \nabla\right]\right) u, \Psi_{j, \mu}^{*} J^{s} \mathbf{C} u\right\rangle \mid d t \\
+\int_{0}^{T}\left|\left\langle J^{s}\left[\mathbf{C}, T_{b_{2}} . \nabla\right] \bar{u}, \Psi_{j, \mu}^{*} J^{s} \mathbf{C} u\right\rangle\right| d t .
\end{gathered}
$$

Indeed, since the operators $\Psi_{j, \mu} J^{s}\left[\mathbf{C}, C_{1}\right] J^{-s}$ and $\Psi_{j, \mu} J^{s}\left[\mathbf{C}, C_{2}\right] J^{-s}$ are bounded in $L^{2}$ (and so is $J^{s} \mathbf{C} J^{-s}$ ), the corresponding terms are easily estimated by

$$
A T \sup _{\mu} \nu_{N}\left(\varphi_{1, \mu}\right) \sup _{0 \leq t \leq T}\|u(t)\|_{s}^{2}
$$

We need now for the other terms the following simple lemma :

Lemma 6.1 If $a \in S_{0,0}^{m}$, then, for any real $s$,

$$
J^{s} a(x, D) J^{-s}=a(x, D)+e(x, D)
$$

where $e \in S_{0,0}^{m-1}$ and the semi-norms of $e$ are bounded by those of a.

Proof : It suffices to apply the pseudodifferential calculus and to remark that

$$
e(x, \xi)=\frac{1}{(2 \pi)^{n}} \sum_{j=1}^{n} \int_{0}^{1} \int \mathrm{e}^{-i y \eta} \partial_{\xi_{j}}\left(\langle\xi+t \eta\rangle^{s}\right) \partial_{x_{j}} a(x+y, \xi)\langle\xi\rangle^{-s} d y d \eta d t
$$


We apply the lemma successively with $a(x, D)=i[\mathbf{C}, \mathscr{L}]+\mathbf{C} T_{i b_{1}^{\prime \prime}} \nabla, a(x, D)=\left[\mathbf{C}, T_{b_{1}^{\prime}} \cdot \nabla\right]$ and $a(x, D)=\left[\mathbf{C}, T_{b_{2}} . \nabla\right]$. Since here $m=1$, we obtain that at each time the operator $e(x, D)$ is bounded in $L^{2}$ and that its operator norm is estimated by the semi-norms of $a$. Next, it follows from the pseudodifferential calculus that $\Psi_{j, \mu}^{*} \in \mathrm{Op} S_{1,0}^{0}$ and their seminorms are uniformly bounded with respect to $\mu$, and, consequently, also that $\Psi_{j, \mu}^{*} J^{s} \mathbf{C} J^{-s} \in$ $\mathrm{Op} S_{0,0}^{0}$ and their semi-norms are uniformly estimated by those of $\mathbf{C}$. Hence, the integrals corresponding to the operators $e(x, D)$ are easily estimated by

$$
A R T \sup _{k, \mu} \nu_{N}\left(\varphi_{k, \mu}\right) \sup _{0 \leq t \leq T}\|u(t)\|_{s}^{2}
$$

Thus, it remains to estimate the sum

$$
\begin{gathered}
\int_{0}^{T}\left|\left\langle\left(i[\mathbf{C}, \mathscr{L}]+\mathbf{C} T_{i b_{1}^{\prime \prime}} \nabla\right) J^{s} u, \Psi_{j, \mu}^{*} J^{s} \mathbf{C} u\right\rangle\right| d t+\int_{0}^{T}\left|\left\langle\left[\mathbf{C}, T_{b_{1}^{\prime}} \cdot \nabla\right]\right) J^{s} u, \Psi_{j, \mu}^{*} J^{s} \mathbf{C} u\right\rangle \mid d t \\
+\int_{0}^{T}\left|\left\langle\left[\mathbf{C}, T_{b_{2}} . \nabla\right] J^{s} \bar{u}, \Psi_{j, \mu}^{*} J^{s} \mathbf{C} u\right\rangle\right| d t
\end{gathered}
$$

to which we apply Lemma 5.2 with $S=\Psi_{j, \mu}^{*} J^{s} \mathbf{C} J^{-s}$. We obtain eventually

$$
\begin{aligned}
\sum_{j=1}^{4} \sup _{\mu} \int_{0}^{T}\left|\left\langle\Psi_{j, \mu} J^{s} \tilde{f}, J^{s} \mathbf{C} u\right\rangle\right| d t \leq & \sum_{j=1}^{4} \sup _{\mu} \int_{0}^{T}\left|\left\langle\Psi_{j, \mu} J^{s} \mathbf{C} f, J^{s} \mathbf{C} u\right\rangle\right| d t \\
& +A \sup _{k, \mu} \nu_{N}\left(\varphi_{k, \mu}\right)\left(R T \sup _{[0, T]}\|u\|_{s}^{2}+\frac{1}{R}\left\|J^{s+\frac{1}{2}} u\right\|_{T}^{2}\right)
\end{aligned}
$$

which, together with (62), implies the second estimate of Proposition 3.2. 


\section{Appendix}

\subsection{Proof of Lemma 3.1 :}

We need the following general estimate :

Lemma 7.1 Let $b$ satisfy

$$
\begin{cases}b(x, \xi)=\sum_{\mu \in \mathbb{Z}^{n}} \alpha_{\mu} \varphi_{\mu}(x, \xi), & \sum_{\mu}\left|\alpha_{\mu}\right| \leq A_{0}, \\ \operatorname{supp}\left(x \mapsto \varphi_{\mu}(x, \xi)\right) \subseteq Q_{\mu}^{*}, & \sup _{\mu} \sup _{|\beta| \leq N_{0}}\left\|\langle\xi\rangle^{|\beta|} \partial_{\xi}^{\beta} \varphi_{\mu}\right\|_{L^{\infty}}<\infty,\end{cases}
$$

where $N_{0}$ is a sufficiently large integer, and let $\tilde{u}$ stand for $u$ or $\bar{u}$. Then, there exist $N \in \mathbb{N}$ and $A>0$ such that, for all $T>0$ and every $S_{1}=s_{1}(x, D), S_{2}=s_{2}(x, D)$ in $\mathrm{Op} S_{0,0}^{0}$, we have

$$
\int_{0}^{T}\left|\left\langle S_{1} J^{s} T_{b} J^{-s} \nabla \tilde{u}, S_{2} u\right\rangle\right| d t \leq A\left\|s_{1}\right\|_{C^{N}}\left\|s_{2}\right\|_{C^{N}} \sup _{\mu} \sup _{|\beta| \leq N}\left\|\langle\xi\rangle^{|\beta|} \partial_{\xi}^{\beta} \varphi_{\mu}\right\|_{L^{\infty}}\left\|J^{\frac{1}{2}} u\right\|_{T}^{2} .
$$

Proof : One can write

$$
\begin{gathered}
\left\langle S_{1} J^{s} T_{b} J^{-s} \nabla \tilde{u}, S_{2} u\right\rangle=\sum_{\mu} \alpha_{\mu}\left\langle S_{1} J^{s} T_{\varphi_{\mu}} J^{-s} \nabla \tilde{u}, S_{2} u\right\rangle=\sum_{\mu} \alpha_{\mu}\left\langle S_{2}^{*} S_{1} J^{s} T_{\varphi_{\mu}} J^{-s} \nabla \tilde{u}, u\right\rangle \\
=\sum_{\mu} \alpha_{\mu}\left\langle\langle x-\mu\rangle^{\sigma_{0}} J^{-1 / 2} S_{2}^{*} S_{1} J^{s} T_{\varphi_{\mu}} J^{-s} \nabla J^{-1 / 2}\langle x-\mu\rangle^{\sigma_{0}}\langle x-\mu\rangle^{-\sigma_{0}} J^{1 / 2} \tilde{u},\langle x-\mu\rangle^{-\sigma_{0}} J^{1 / 2} u\right\rangle \\
=\sum_{\mu} \alpha_{\mu}\left\langle S_{\mu}\langle x-\mu\rangle^{\sigma_{0}} T_{\varphi_{\mu}}\langle x-\mu\rangle^{\sigma_{0}} J_{\mu} \tilde{u}_{\mu}, u_{\mu}\right\rangle
\end{gathered}
$$

where $\quad S_{\mu}=\langle x-\mu\rangle^{\sigma_{0}} J^{-1 / 2} S_{2}^{*} S_{1} J^{s}\langle x-\mu\rangle^{-\sigma_{0}}, \quad J_{\mu}=\langle x-\mu\rangle^{-\sigma_{0}} J^{-s} \nabla J^{-1 / 2}\langle x-\mu\rangle^{\sigma_{0}}$ and $u_{\mu}=\langle x-\mu\rangle^{-\sigma_{0}} J^{1 / 2} u$. Now, it follows from the pseudodifferential calculus (Theorem 2.1) and from Lemma 2.1 that $S_{\mu}$ and $J_{\mu}$ are in $\mathrm{Op} S_{0,0}^{s-1 / 2}$ and $\mathrm{Op} S_{0,0}^{1 / 2-s}$ respectively, and that we can estimate their semi-norms uniformly in $\mu$. Next, it follows from Lemma 2.4 that the operator norm of $\langle x-\mu\rangle^{\sigma_{0}} T_{\varphi_{\mu}}\langle x-\mu\rangle^{\sigma_{0}}$ acting in $H^{s-1 / 2}\left(\mathbb{R}^{n}\right)$ is estimated by $\sup _{|\beta| \leq N}\left\|\langle\xi\rangle^{|\beta|} \partial_{\xi}^{\beta} \varphi_{\mu}\right\|_{L^{\infty}}$ uniformly in $\mu$. Hence, the application of Cauchy-Schwarz inequality and Calderon-Vaillancourt's theorem allows us to obtain

$$
\begin{gathered}
\int_{0}^{T}\left|\left\langle S_{1} J^{s} T_{b} J^{-s} \nabla \tilde{u}, S_{2} u\right\rangle\right| d t \\
\leq \sum_{\mu}\left|\alpha_{\mu}\right|\left\|S_{\mu}\right\|_{\mathscr{L}\left(H^{s-1 / 2}, L^{2}\right)}\left\|\langle x-\mu\rangle^{\sigma_{0}} T_{\varphi_{\mu}}\langle x-\mu\rangle^{\sigma_{0}}\right\|_{\mathscr{L}\left(H^{s-1 / 2}\right)}\left\|J_{\mu}\right\|_{\mathscr{L}\left(L^{2}, H^{s-1 / 2}\right)} \int_{0}^{T}\left\|u_{\mu}\right\|_{0}^{2} d t \\
\leq A\left\|s_{1}\right\|_{C^{N}}\left\|s_{2}\right\|_{C^{N}} \sup _{\mu} \sup _{|\beta| \leq N}\left\|\langle\xi\rangle^{|\beta|} \partial_{\xi}^{\beta} \varphi_{\mu}\right\|_{L^{\infty}}\left\|J^{\frac{1}{2}} u\right\|_{T}^{2},
\end{gathered}
$$

which proves the lemma. 
Now, let us write $T_{b_{k}-b_{k, m}}=T_{b_{k}-b_{k, m^{\prime}}}+T_{b_{k, m^{\prime}}-b_{k, m}}$ and apply Lemma 7.1 first to $b=$ $b_{k}-b_{k, m^{\prime}}$ with $S_{1}=S_{2}=\mathbf{C}_{m}$. We obtain

$$
\begin{gathered}
\int_{0}^{T}\left|\left\langle\mathbf{C}_{m} J^{s} T_{b_{k}-b_{k, m^{\prime}}} \nabla \tilde{u}, \mathbf{C}_{m} J^{s} u\right\rangle\right| d t=\int_{0}^{T}\left|\left\langle\mathbf{C}_{m} J^{s} T_{b_{k}-b_{k, m^{\prime}}} J^{-s} \nabla \tilde{v}, \mathbf{C}_{m} v\right\rangle\right| d t \\
\leq A \sup _{\mu} \nu_{N}\left(\varphi_{1, \mu, m}\right)^{2} \sup _{\mu} \sup _{|\beta| \leq N}\left\|\langle\xi\rangle^{|\beta|} \partial_{\xi}^{\beta}\left(\varphi_{k, \mu}-\varphi_{k, \mu, m^{\prime}}\right)\right\|_{L^{\infty}}\left\|J^{\frac{1}{2}} v\right\|_{T}^{2}, \\
\leq A \frac{m^{2 N^{2}}}{m^{\prime \sigma}} \sup _{\mu} \sup _{|\beta| \leq N}\left\|\langle\xi\rangle^{|\beta|} \partial_{\xi}^{\beta} \varphi_{k, \mu}\right\|_{C^{\sigma}}\left\|J^{\frac{1}{2}+s} u\right\|_{T}^{2} \leq A \frac{m^{2 N^{2}}}{m^{\prime \sigma}}\left\|J^{\frac{1}{2}+s} u\right\|_{T}^{2},
\end{gathered}
$$

where $v=J^{s} u$ and $\sigma=\inf \{\varrho, 1\}$. As for the study of the other term, we write

$$
\begin{gathered}
\left\langle\mathbf{C}_{m} J^{s} T_{b_{k, m^{\prime}}-b_{k, m}} \nabla \tilde{u}, \mathbf{C}_{m} J^{s} u\right\rangle=\left\langle\mathbf{C}_{m} J^{s} T_{b_{k, m^{\prime}}-b_{k, m}} J^{-s} \nabla \tilde{v}, \mathbf{C}_{m} v\right\rangle \\
=\left\langle J^{s} T_{b_{k, m^{\prime}}-b_{k, m}} J^{-s} \nabla \mathbf{C}_{m} \tilde{v}, \mathbf{C}_{m} v\right\rangle+\left\langle\left[\mathbf{C}_{m}, J^{s} T_{b_{k, m^{\prime}}-b_{k, m}} J^{-s} \nabla\right] \tilde{v}, \mathbf{C}_{m} v\right\rangle,
\end{gathered}
$$

and then apply Lemma 5.2(iii) to the second term in (64) to obtain

$$
\begin{aligned}
& \int_{0}^{T}\left|\left\langle\left[\mathbf{C}_{m}, J^{s} T_{b_{k, m^{\prime}}-b_{k, m}} J^{-s} \nabla\right] \tilde{v}, \mathbf{C}_{m} v\right\rangle\right| d t \\
& \leq A \sup _{\mu} \nu_{N}\left(\varphi_{1, \mu, m}\right)^{2} \sup _{\mu}\left\|\varphi_{k, \mu, m^{\prime}}-\varphi_{k, \mu, m}\right\|_{C^{N}}\left(T \sup _{[0, T]}\|v\|_{0}^{2}+\frac{1}{R}\left\|J^{\frac{1}{2}} v\right\|_{T}^{2}\right)
\end{aligned}
$$

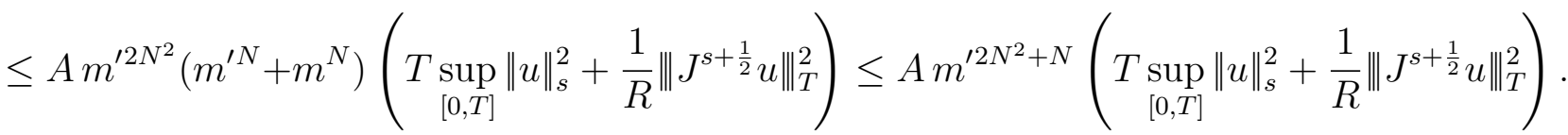

Finally, recalling that $\mathbf{C}_{m} \bar{u}=\overline{\mathbf{C}_{m} u}$ and applying Lemma 7.1 to the first term in (64) with $S_{1}=S_{2}=I d$, we get

$$
\begin{gathered}
\int_{0}^{T}\left|\left\langle J^{s} T_{b_{k, m^{\prime}}-b_{k, m}} J^{-s} \nabla \mathbf{C}_{m} \tilde{v}, \mathbf{C}_{m} v\right\rangle\right| d t \leq A \operatorname{supsup}_{\mu|\beta| \leq N}\left\|\langle\xi\rangle^{|\beta|} \partial_{\xi}^{\beta}\left(\varphi_{k, \mu, m^{\prime}}-\varphi_{k, \mu, m}\right)\right\|_{L^{\infty}}\left\|J^{\frac{1}{2}} \mathbf{C}_{m} v\right\|_{T}^{2} \\
\leq A\left(\operatorname{supsup}_{\mu|\beta| \leq N}\left\|\langle\xi\rangle^{|\beta|} \partial_{\xi}^{\beta}\left(\varphi_{k, \mu, m^{\prime}}-\varphi_{k, \mu}\right)\right\|_{L^{\infty}}+\operatorname{supsup}_{\mu|\beta| \leq N}\left\|\langle\xi\rangle^{|\beta|} \partial_{\xi}^{\beta}\left(\varphi_{k, \mu}-\varphi_{k, \mu, m}\right)\right\|_{L^{\infty}}\right)\left\|J^{\frac{1}{2}} \mathbf{C}_{m} v\right\|_{T}^{2} \\
\leq\left(\frac{A}{m^{\prime \sigma}}+\frac{A}{m^{\sigma}}\right) \sup _{\mu} \sup _{|\beta| \leq N}\left\|\langle\xi\rangle^{|\beta|} \partial_{\xi}^{\beta} \varphi_{k, \mu}\right\|_{C^{\sigma}}\left\|J^{\frac{1}{2}} \mathbf{C}_{m} v\right\|_{T}^{2} \\
\leq\left(\frac{A}{m^{\prime \sigma}}+\frac{A}{m^{\sigma}}\right)\left\|J^{\frac{1}{2}} \mathbf{C}_{m} v\right\|_{T}^{2} \leq \frac{A}{m^{\sigma}}\left\|J^{\frac{1}{2}} \mathbf{C}_{m} v\right\|_{T}^{2} .
\end{gathered}
$$

It remains to compare $\left\|J^{\frac{1}{2}} \mathbf{C}_{m} v\right\|_{T}^{2}=\left\|J^{\frac{1}{2}} \mathbf{C}_{m} J^{s} u\right\|_{T}^{2}$ with $\left\|J^{s+\frac{1}{2}} \mathbf{C}_{m} u\right\|_{T}^{2}$. Of course, one can write $J^{\frac{1}{2}} \mathbf{C}_{m} J^{s} u=J^{s+\frac{1}{2}} J^{-s} \mathbf{C}_{m} J^{s} u$ and it follows from Lemma 6.1 that $J^{-s} \mathbf{C}_{m} J^{s}-\mathbf{C}_{m}=E_{m}$ is in $\mathrm{Op} S_{0,0}^{-1}$ and the semi-norms of $E_{m}$ are bounded by those of $\mathbf{C}_{m}$. Hence, since $J^{s+\frac{1}{2}} E_{m} J^{-s}$ is in $\mathrm{Op} S_{0,0}^{-1 / 2}$,

$$
\left\|J^{s+\frac{1}{2}} E_{m} u\right\|_{T}^{2}=\sup _{\mu} \int_{0}^{T} \int\left|\langle x-\mu\rangle^{-\sigma_{0}} J^{s+\frac{1}{2}} E_{m} u\right|^{2} d x d t \leq \int_{0}^{T} \int\left|J^{s+\frac{1}{2}} E_{m} u\right|^{2} d x d t
$$




$$
\begin{aligned}
& \leq A \sup _{\mu} \nu_{N}\left(\varphi_{1, \mu, m}\right)^{2} \int_{0}^{T} \int\left|J^{s} u\right|^{2} d x d t \leq A T m^{2 N^{2}} \sup _{[0, T]}\|u\|_{s}^{2} \\
& \text { and } \\
& \qquad\left\|J^{\frac{1}{2}} \mathbf{C}_{m} v\right\|_{T}^{2} \leq 2\left\|J^{s+\frac{1}{2}} \mathbf{C}_{m} u\right\|_{T}^{2}+2 A T m^{2 N^{2}} \sup _{[0, T]}\|u\|_{s}^{2},
\end{aligned}
$$

which implies that

$$
\int_{0}^{T}\left|\left\langle J^{s} T_{b_{k, m^{\prime}}-b_{k, m}} J^{-s} \nabla \mathbf{C}_{m} \tilde{v}, \mathbf{C}_{m} v\right\rangle\right| d t \leq \frac{A}{m^{\sigma}}\left\|J^{s+\frac{1}{2}} \mathbf{C}_{m} u\right\|_{T}^{2}+A T m^{2 N^{2}} \sup _{[0, T]}\|u\|_{s}^{2},
$$

where, of course, the constant $A$ has changed. Summing up, we have proven that

$$
\begin{gathered}
\int_{0}^{T}\left|\left\langle\mathbf{C}_{m} J^{s} T_{b_{k}-b_{k, m}} \nabla \tilde{u}, \mathbf{C}_{m} J^{s} u\right\rangle\right| d t \\
\leq \frac{A m^{2 N^{2}}}{m^{\prime \sigma}}\left\|J^{s+\frac{1}{2}} u\right\|_{T}^{2}+A m^{\prime 2 N^{2}+N}\left(\underset{[0, T]}{\left.T \sup _{[0,}\|u\|_{s}^{2}+\frac{1}{R}\left\|J^{s+\frac{1}{2}} u\right\|_{T}^{2}\right)+\frac{A}{m^{\sigma}}\left\|J^{s+\frac{1}{2}} \mathbf{C}_{m} u\right\|_{T}^{2}} ;\right.
\end{gathered}
$$

that is, we have proven Lemma 3.1.

\subsection{Anisotropic Sobolev spaces}

There are several notions of anisotropic Sobolev space in the literature. However, we have not been able to find a reference with the results we need in this paper. Therefore, we are going to define our spaces and next prove the results we need.

We denote by $(x, y)$ the variable in $\mathbb{R}^{n} \times \mathbb{R}^{n^{\prime}}$ and by $(\xi, \eta)$ its Fourier dual variable.

Definition 7.1 If $s, s^{\prime} \in \mathbb{R}$, we denote by $H^{s, s^{\prime}}\left(\mathbb{R}^{n} \times \mathbb{R}^{n^{\prime}}\right)$ the space of tempered distributions $u$ in $\mathbb{R}^{n} \times \mathbb{R}^{n^{\prime}}$ such that the integral

$$
\int_{\mathbb{R}^{n} \times \mathbb{R}^{n^{\prime}}}\langle\xi\rangle^{2 s}\langle\eta\rangle^{2 s^{\prime}}|\hat{u}(\xi, \eta)|^{2} d \xi d \eta
$$

is finite.

We call this space an anisotropic Sobolev space. Note that this is different, for example, from the classical space $H^{r, s}$ of Lions and Magenes, [LiMa], Vol 2. Clearly, $H^{s, s^{\prime}}\left(\mathbb{R}^{n} \times \mathbb{R}^{n^{\prime}}\right)$ is a Hilbert space when it is provided with the obvious scalar product. We also denote by $\|u\|_{s, s^{\prime}}$ the norm of $u$ in this space and, of course, it is equal to the square root of (66).

Note also that the space $H^{s, s^{\prime}}\left(\mathbb{R}^{n} \times \mathbb{R}^{n^{\prime}}\right)$ in the above definition coincides with the space $H^{s}\left(\mathbb{R}^{n}, H^{s^{\prime}}\left(\mathbb{R}^{n^{\prime}}\right)\right)$ and, by symmetry, with $H^{s^{\prime}}\left(\mathbb{R}^{n^{\prime}}, H^{s}\left(\mathbb{R}^{n}\right)\right)$.

In this paper, we need the following two results on anisotropic Sobolev spaces. The first one is the Sobolev injection :

Proposition 7.1 If $s>\frac{n}{2}$ and $s^{\prime}>\frac{n^{\prime}}{2}$, then, $H^{s, s^{\prime}}\left(\mathbb{R}^{n} \times \mathbb{R}^{n^{\prime}}\right) \subset L^{\infty}\left(\mathbb{R}^{n} \times \mathbb{R}^{n^{\prime}}\right)$ with continuous injection. 
Proof: If $u \in H^{s, s^{\prime}}$, then, $\hat{u}(\xi, \eta)=\langle\xi\rangle^{-s}\langle\eta\rangle^{-s^{\prime}} \cdot\langle\xi\rangle^{s}\langle\eta\rangle^{s^{\prime}} \hat{u}(\xi, \eta)$; hence, $\hat{u} \in L^{2} . L^{2} \subset L^{1}$ and $\|u\|_{L^{\infty}} \leq C\|\hat{u}\|_{L^{1}} \leq C^{\prime}\|u\|_{s, s^{\prime}}$, where $C$ and $C^{\prime}$ are constants which are independent of $u$.

The other result is an interpolation inequality :

Proposition 7.2 If $s=(1-\theta) s_{1}+\theta s_{2}$ and $s^{\prime}=(1-\theta) s_{1}^{\prime}+\theta s_{2}^{\prime}$, where $\theta \in[0,1]$, $s_{1}, s_{2}, s_{1}^{\prime}, s_{2}^{\prime} \in \mathbb{R}$, then, for any $u \in H^{s_{1}, s_{1}^{\prime}}\left(\mathbb{R}^{n} \times \mathbb{R}^{n^{\prime}}\right) \cap H^{s_{2}, s_{2}^{\prime}}\left(\mathbb{R}^{n} \times \mathbb{R}^{n^{\prime}}\right)$, we have

$$
\|u\|_{s, s^{\prime}} \leq\|u\|_{s_{1}, s_{1}^{\prime}}^{1-\theta}\|u\|_{s_{2}, s_{2}^{\prime}}^{\theta}
$$

Proof: Indeed, we have

$$
\begin{gathered}
\|u\|_{s, s^{\prime}}^{2}=\int_{\mathbb{R}^{n} \times \mathbb{R}^{n^{\prime}}}\langle\xi\rangle^{2(1-\theta) s_{1}+2 \theta s_{2}}\langle\eta\rangle^{2(1-\theta) s_{1}^{\prime}+2 \theta s_{2}^{\prime}}|\hat{u}(\xi, \eta)|^{2} d \xi d \eta \\
=\int_{\mathbb{R}^{n} \times \mathbb{R}^{n^{\prime}}}\left(\langle\xi\rangle^{s_{1}}\langle\eta\rangle^{s_{1}^{\prime}}|\hat{u}(\xi, \eta)|\right)^{2(1-\theta)}\left(\langle\xi\rangle^{s_{2}}\langle\eta\rangle^{s_{2}^{\prime}}|\hat{u}(\xi, \eta)|\right)^{2 \theta} d \xi d \eta \\
\leq\left(\int_{\mathbb{R}^{n} \times \mathbb{R}^{n^{\prime}}}\langle\xi\rangle^{2 s_{1}}\langle\eta\rangle^{2 s_{1}^{\prime}}|\hat{u}(\xi, \eta)|^{2} d \xi d \eta\right)^{1-\theta}\left(\int_{\mathbb{R}^{n} \times \mathbb{R}^{n^{\prime}}}\langle\xi\rangle^{2 s_{2}}\langle\eta\rangle^{2 s_{2}^{\prime}}|\hat{u}(\xi, \eta)|^{2} d \xi d \eta\right)^{\theta}=\|u\|_{s_{1}, s_{1}^{\prime}}^{2(1-\theta)}\|u\|_{s_{2}, s_{2}^{\prime}}^{2 \theta},
\end{gathered}
$$

where we have applied Hölder's inequality.

Actually, we need the above results for anisotropic Sobolev spaces on domains $\Omega$ in $\mathbb{R}^{n} \times \mathbb{R}^{n^{\prime}}$, and since the theory of such spaces is less simple, we shall restrict ourselves to the case that arises in this paper, that is, the case $\Omega=I \times \mathbb{R}^{n}$ where $I$ is a bounded interval in $\mathbb{R}$, and only to the case $s \geq 0$. First, let us set, by definition,

$$
H^{s, s^{\prime}}(\Omega)=H^{s}\left(I, H^{s^{\prime}}\left(\mathbb{R}^{n}\right)\right)
$$

in the sense that $u(x, y)$ is in $H^{s, s^{\prime}}(\Omega)$ if and only if

$$
\partial_{x}^{\alpha} J_{y}^{s^{\prime}} u \in L^{2}(\Omega) \text { for }|\alpha| \leq s
$$

and $\quad \int_{I \times I \times \mathbb{R}^{n}} \frac{\left|\partial_{x}^{\alpha} J_{y}^{s^{\prime}} u(x, y)-\partial_{x}^{\alpha} J_{y}^{s^{\prime}} u\left(x^{\prime}, y\right)\right|^{2}}{\left|x-x^{\prime}\right|^{1+2 \sigma}} d x d x^{\prime} d y<\infty \quad$ if $\quad 0<\sigma=s-[s]<1$.

Of course, the norm in this space is defined by

$$
\|u\|_{s, s^{\prime}, \Omega}^{2}=\sum_{|\alpha| \leq s}\left\|\partial_{x}^{\alpha} J_{y}^{s^{\prime}} u\right\|_{L^{2}(\Omega)}^{2} \quad \text { if } \quad s \in \mathbb{N}
$$

and $\|u\|_{s, s^{\prime}, \Omega}^{2}=\sum_{|\alpha| \leq[s]}\left\|\partial_{x}^{\alpha} J_{y}^{s^{\prime}} u\right\|_{L^{2}(\Omega)}^{2}+\int_{I \times I \times \mathbb{R}^{n}} \frac{\left|\partial_{x}^{\alpha} J_{y}^{s^{\prime}} u(x, y)-\partial_{x}^{\alpha} J_{y}^{s^{\prime}} u\left(x^{\prime}, y\right)\right|^{2}}{\left|x-x^{\prime}\right|^{1+2 \sigma}} d x d x^{\prime} d y$ otherwise.

Now, we can prove for $H^{s, s^{\prime}}(\Omega)$ the results analogous to the above ones. 
Proposition 7.3 (i) If $s>\frac{1}{2}$ and $s^{\prime}>\frac{n}{2}$, then, $H^{s, s^{\prime}}(\Omega) \subset L^{\infty}(\Omega)$ with continuous injection.

(ii) If $s=(1-\theta) s_{1}+\theta s_{2}$ and $s^{\prime}=(1-\theta) s_{1}^{\prime}+\theta s_{2}^{\prime}$, where $\theta \in[0,1], s_{1} \geq 0, s_{2} \geq$ $0, s_{1}^{\prime}, s_{2}^{\prime} \in \mathbb{R}$, then, there exists a constant $C$ such that, for any $u \in H^{s_{1}, s_{1}^{\prime}}(\Omega) \cap H^{s_{2}, s_{2}^{\prime}}(\Omega)$, we have

$$
\|u\|_{s, s^{\prime}, \Omega} \leq C\|u\|_{s_{1}, s_{1}^{\prime}, \Omega}^{1-\theta}\|u\|_{s_{2}, s_{2}^{\prime}, \Omega}^{\theta}
$$

Proof: Since we can not use directly the Fourier transformation, the idea is to construct a bounded linear extension operator

$$
P_{\Omega}: H^{s, s^{\prime}}(\Omega) \rightarrow H^{s, s^{\prime}}\left(\mathbb{R} \times \mathbb{R}^{n}\right),
$$

that is, which satisfies $\left.P_{\Omega} u\right|_{\Omega}=u$, for all $u \in H^{s, s^{\prime}}(\Omega)$. Indeed, assume that such a $P_{\Omega}$ exists. Then, for $u \in H^{s, s^{\prime}}(\Omega)$ with $s>\frac{1}{2}$ and $s^{\prime}>\frac{n}{2}$,

$$
\|u\|_{L^{\infty}(\Omega)}=\left\|P_{\Omega} u\right\|_{L^{\infty}(\Omega)} \leq\left\|P_{\Omega} u\right\|_{L^{\infty}\left(\mathbb{R} \times \mathbb{R}^{n}\right)} \leq C\left\|P_{\Omega} u\right\|_{s, s^{\prime}} \leq C^{\prime}\|u\|_{s, s^{\prime}, \Omega},
$$

where we have applied Proposition 7.1 and the boundedness of $P_{\Omega}$, and this proves $(i)$.

Furthermore, under the assumptions of $(i i)$, we have

$$
\|u\|_{s, s^{\prime}, \Omega}=\left\|P_{\Omega} u\right\|_{s, s^{\prime}, \Omega} \leq\left\|P_{\Omega} u\right\|_{s, s^{\prime}, \mathbb{R} \times \mathbb{R}^{n}}
$$

and it is a classical fact that there exists a constant $C$ such that, for all $v \in H^{s}\left(\mathbb{R}^{d}\right)$,

$$
\sum_{|\alpha| \leq[s]}\left\|\partial^{\alpha} v\right\|_{L^{2}\left(\mathbb{R}^{d}\right)}^{2}+\int_{\mathbb{R}^{d} \times \mathbb{R}^{d}} \frac{\left|\partial^{\alpha} v(x)-\partial^{\alpha} v\left(x^{\prime}\right)\right|^{2}}{\left|x-x^{\prime}\right|^{d+2 \sigma}} d x d x^{\prime} \leq C\|v\|_{s}^{2}
$$

now, applying this inequality to $v(x)=J_{y}^{s^{\prime}} P_{\Omega} u(x, y)$, with $d=1$, and integrating with respect to $y$ gives

$$
\left\|P_{\Omega} u\right\|_{s, s^{\prime}, \mathbb{R} \times \mathbb{R}^{n}}^{2} \leq C\left\|P_{\Omega} u\right\|_{s, s^{\prime}}^{2}
$$

Finally, applying Proposition 7.2 and the boundedness of $P_{\Omega}$ yields

$$
\|u\|_{s, s^{\prime}, \Omega} \leq \sqrt{C}\left\|P_{\Omega} u\right\|_{s, s^{\prime}} \leq \sqrt{C}\left\|P_{\Omega} u\right\|_{s_{1}, s_{1}^{\prime}}^{1-\theta}\left\|P_{\Omega} u\right\|_{s_{2}, s_{2}^{\prime}}^{\theta} \leq C^{\prime}\|u\|_{s_{1}, s_{1}^{\prime}, \Omega}^{1-\theta}\|u\|_{s_{2}, s_{2}^{\prime}, \Omega}^{\theta},
$$

which establishes $(i i)$.

It remains to construct $P_{\Omega}$ as in (67). In fact, the classical theory of Sobolev spaces already provides a bounded linear extension operator

$$
P_{I}: H^{s}(I) \rightarrow H^{s}(\mathbb{R})
$$

such that $\left.P_{I} u\right|_{I}=u$, for all $u \in H^{s}(I)$. If $u \in H^{s, s^{\prime}}(\Omega)$, let us set

$$
P_{\Omega} u(x, y)=\left(P_{I}\right)_{x} u(x, y)
$$

Clearly, this defines a linear operator such that $\left.P_{\Omega} u\right|_{\Omega}=u$. Let us show the boundedness of $P_{\Omega}: H^{s, s^{\prime}}(\Omega) \rightarrow H^{s, s^{\prime}}\left(\mathbb{R} \times \mathbb{R}^{n}\right)$. It follows from the definition that $x \mapsto J_{y}^{s^{\prime}} u(x, y)$ is in 
the Sobolev space $H^{s}(I)$ for almost all $y \in \mathbb{R}^{n}$. Hence, $x \mapsto\left(P_{I}\right)_{x} J_{y}^{s^{\prime}} u(x, y)$ is in $H^{s}(\mathbb{R})$ for almost all $y \in \mathbb{R}^{n}$ and there exists a constant $C$ which depends neither on $u$ nor on $y$ such that

$$
\left\|\left(P_{I}\right)_{x} J_{y}^{s^{\prime}} u(x, y)\right\|_{H^{s}(\mathbb{R})} \leq C\left\|J_{y}^{s^{\prime}} u(x, y)\right\|_{H^{s}(I)}, \text { for a.e. } y \in \mathbb{R}^{n} .
$$

Since $\left(P_{I}\right)_{x} J_{y}^{s^{\prime}} u=J_{y}^{s^{\prime}} P_{\Omega} u$, this inequality can be written more explicitly as

$$
\begin{aligned}
& \int_{\mathbb{R}}\left|J_{x}^{s} J_{y}^{s^{\prime}} P_{\Omega} u(x, y)\right|^{2} d x \leq C^{2} \sum_{|\alpha| \leq[s]} \int_{I}\left|\partial_{x}^{\alpha} J_{y}^{s^{\prime}} u(x, y)\right|^{2} d x \\
& +C^{2} \int_{I \times I} \frac{\left|\partial_{x}^{\alpha} J_{y}^{s^{\prime}} u(x, y)-\partial_{x}^{\alpha} J_{y}^{s^{\prime}} u\left(x^{\prime}, y\right)\right|^{2}}{\left|x-x^{\prime}\right|^{1+2 \sigma}} d x d x^{\prime} \text { for a.e. } y \in \mathbb{R}^{n} .
\end{aligned}
$$

Integrating over $\mathbb{R}^{n}$ with respect to $y$ gives

$$
\left\|P_{\Omega} u\right\|_{s, s^{\prime}}^{2} \leq C^{2}\|u\|_{s, s^{\prime}, \Omega}^{2}
$$

which proves the boundedness of $P_{\Omega}$ and achieves the proof of the proposition.

\section{References}

[Bie] P.-Y. Bienaimé, Existence locale et effet régularisant précisés pour des équations non linéaires de type Schrödinger, Rev. Mat. Iberoam. 30 (2014), no. 3, 751-798.

[KePoVe] C. E. Kenig, G. Ponce, L. Vega, Smoothing effect and local existence theory for the generalized nonlinear Schrödinger equation, Inventiones Mathematicae 134, 489-545 (1998).

[Tak] J. Takeuchi, Le problème de Cauchy pour certaines équations aux derivées partielles du type de Schrödinger; symétrisations indépendantes du temps, C. R. Acad. Sci. Paris, t315, Série 1, 1055-1058 (1992).

[Bon] J.-M. Bony : Calcul symbolique et propagation des singularités pour les équations aux dérivées partielles non linéaires, Annales scientifiques de l'E.N.S, $4^{\mathrm{è}}$ série, tome 14, nº2, p 209-246 (1981).

[BeLo] J. Bergh, J. Löfström : Interpolation spaces, an introduction, Springer Verlag (1976).

[Doi] S. Doi : On the Cauchy problem for Schrödinger type equations and the regularity of the solutions, J. Math. Kyoto Univ. 34, 319-328 (1994).

[CoMe] R. Coifman et Y. Meyer : Au dela des opérateurs pseudodifférentiels, Asterisque 57, 2ème eedition S.M.F. (1978). 
[Mey] Y. Meyer : Nouvelles estimations pour les solutions d'équations aux dérivées partielles non linéaires, seeminaire Goulaouic-Meyer-Schwartz 1981-1982, Exposee $n^{\circ}$ VI, 10 Novembre 1981.

[Me2] Y. Meyer : Remarques sur un théorème de J.-M. Bony, Supplemento ai Rendi Conti del Circolo Matematico di Palermo, Atti del Seminario di Analisi Armonica, Serie II, Numero 1, 1981.

[Tay] M. E. Taylor : Pseudodifferential Operators and Nonlinear PDE, Birkhäuser (1991).

[Tat] D. Tataru : On the Fefferman-Phong inequality and related problems, Communication in Partial Differential Equations, 27 : 11, 2101-2138 (2002).

[Rob] L. Robbiano : Kato smoothing effect for Schrödinger operator, in "Studies in phase space analysis with applications to PDEs", M. Cicognani, F. Colombini, D. Del Santo Editors, Birkhäuser (2013).

[LiMa] J.-L. Lions, E. Magenes, Problèmes aux limites non homogènes et applications, Dunod (1968).

[KePoVe2] C. E. Kenig, G. Ponce, L. Vega, The Cauchy problem for quasi-linear Schrödinger equations, Invent. Math. 158 (2004), 343-388.

[KePoRoVe1] C. E. Kenig, G. Ponce, C. Rolvung, L. Vega, The general quasilinear ultrahyperbolic Schrödinger equation, Adv. Math. 196, No. 2 (2005), 402-433.

[KePoRoVe2] C. E. Kenig, G. Ponce, C. Rolvung, and L. Vega, Variable coefficient Schrödinger flows for ultrahyperbolic operators, Adv. Math. 206, No. 2 (2006), 373-486.

[MaMeTa1] J. L. Marzuola, J. Metcalfe, D. Tataru, Quasilinear Schrdinger equations I: Small data and quadratic interactions, Advances in Mathematics, 231(2),(2012), 1151-1172. DOI: 10.1016/j.aim.2012.06.010

[MaMeTa2] J. L. Marzuola, J. Metcalfe, D. Tataru, Quasilinear Schrdinger equations, II: Small data and cubic nonlinearities, Kyoto J. Math. 54 (2014), no. 3, 529-546. doi:10.1215/21562261-2693424.

[BeTa] I. Bejenaru, D. Tataru, Large data local solutions for the derivative NLS equation, J. Eur. Math. Soc. 10 (2008), 957985. 